\title{
EXACT NONREFLECTING BOUNDARY CONDITIONS FOR THREE DIMENSIONAL POROELASTIC WAVE EQUATIONS*
}

\author{
WENSHENG ZHANG ${ }^{\dagger}$, LI TONG ${ }^{\dagger}$, AND ERIC T. CHUNG ${ }^{\ddagger}$
}

\begin{abstract}
Simulation of waves in complex poroelastic media is crucial in providing important geophysical information that cannot be obtained via simple elastic or acoustic models. Thus there is a need to design an artificial boundary condition for simulation using the numerical approximation of such a problem. In this paper, our aim is to derive an exact nonreflecting boundary condition for the three dimensional poroelastic wave equations based on the Grote-Keller method. The proposed boundary condition is nonlocal in space, but local in time and can be coupled easily with standard numerical approaches for the computation of numerical solutions. Numerical results computed by the finite difference method demonstrate the effectiveness of our method.
\end{abstract}

Key words. Poroelastic wave equations, wave propagation in porous media, exact nonreflecting boundary conditions, artificial boundary conditions.

AMS subject classifications. 35L05, 35L20, 65M99, 78A40, 78A45.

\section{Introduction}

Simulation of elastic wave propagation in complex poroelastic media is an important research area due to its wide range of applications in reservoir exploration, wave scattering problems, and structural mechanics. For instance, in order to obtain useful insight for the exploration of natural resources such as hydrocarbon, the behavior of elastic waves propagating in fluid-saturated porous media provides the information required. By using a model based on the poroelastic wave equations, the effects of fluid, pressure, porosity, and permeability between phases can be systematically taken into account and produces more accurate solutions that cannot be obtained through the use of pure elastic or acoustic models $[15,16]$. Therefore, there is a need to numerically solve the three dimensional poroelastic wave equations in unbounded regions, and for this aim an artificial boundary condition is needed. It is thus the aim of this paper to derive exact nonreflecting boundary conditions for the three dimensional poroelastic wave equations.

Absorbing boundary conditions (ABCs), also called radiation boundary conditions or nonreflecting boundary conditions, have been widely studied for different types of wave equations (e.g. [5, 22, 26, 33]). Many effective and important methods have emerged (see, e.g., the review papers [24,33]). These methods may be classified into four types.

The first type of ABCs uses a viscous damping boundary [13, 47], where an exponential function is constructed to attenuate the wavefield within a damping layer near the boundary. However, it is generally difficult to find a proper attenuation function to absorb incident waves perfectly. The second type of ABCs is based on one-way wave equations which are imposed on the boundary of the computational

*Received: November 10, 2011; accepted (in revised form): November 27, 2012. Communicated by Olof Runborg.

${ }^{\dagger}$ LSEC, Institute of Computational Mathematics and Scientific/Engineering Computing, Academy of Mathematics and Systems Science, Chinese Academy of Sciences, 100190, Beijing, P.R. China (zws@lsec.cc.ac.cn; tongli@lsec.cc.ac.cn).

This research is supported by State Key Project with grant number 2010 CB731505.

${ }^{\ddagger}$ Department of Mathematics, The Chinese University of Hong Kong, Hong Kong SAR (tschung@ math.cuhk.edu.hk).

The research is partly supported by the Hong Kong RGC General Research Fund (Project: 400411). 
domain to make the boundary transparent to outgoing waves $[17,22,23,40,42$, $43,44]$. The design of this type of $\mathrm{ABCs}$ is related to the approximation of the square root operator with various techniques such as paraxial approximation, Padé approximation, Chebyshev approximation, and least squares approximation [17, 21, $23,40,45,49,56,57]$. Engquist and Majda [22] developed a systematic method for obtaining a hierarchy of local boundary conditions based on approximating the symbol of the governing pseudodifferential operator by rational functions. The ABCs by Higdon [42] annihilate acoustic wave reflections in a finite number of specified directions and are easily extended to higher dimensions. The most popular forms are the first-order and the second-order Engquist-Majda or Higdon ABCs, which are easy to implement but may not be accurate enough. An $\mathrm{ABC}$ involving a third-order or a fourth-order derivative is seldom used, because it requires significant computational time and may result in numerical instabilities $[50,53]$. Many seemingly reasonable approximations turn out to be ill-posed and hence useless. Well-posedness results for the one-way wave equations have been given in $[21,22,40,56]$. Recently highorder $\mathrm{ABCs}$ which are local and involve no higher derivatives are also devised [7,37]. They are based on a high-order form of Higdon ABCs by using auxiliary variables $[24,26,27,36]$.

The third type of ABCs is the perfectly matched layer (PML) proposed originally by Bérenger for the Maxwell's equations [10]. For this method, the computational domain is wrapped by an artificial layer to absorb the outgoing waves. This absorbing layer, called the perfectly matched layer, is constructed so that inside the layer, the solution decays exponentially. Hence if the layer is sufficiently wide the solution will be close to zero at the outer boundary. The stability analysis of PML are also investigated $[4,6,8,21]$. The PML method has been proved to work very well for a wide range of incident angles and frequencies. It has been widely used for electromagnetic and elastic wave equations (e.g. [11, 18, 41, 48]), poroelastic elastic equations [58, 59], and the mixed hyperbolic-parabolic systems [3]. The comparison of high-order absorbing boundary conditions and PML can be found in [52].

Many boundary conditions mentioned above such as the well-known EngquistMajda ABCs [17] and the Higdon ABCs [42, 45] are local differential operators on boundaries. In fact, Higdon [44] showed that any local boundary condition involving a differential operator eliminates boundary reflections at certain angles of incidence but not the others. It is then necessary to define the artificial boundary far from the region of interest, or to use a thick absorbing layer, to reduce the amount of reflection in order to achieve high accuracy, and this procedure will result in a more computationally expensive method.

The fourth type of ABCs is the exact nonreflecting boundary conditions on a spherical computational domain. Contrary to other ABCs, this type of method is nonlocal in space. It was first proposed by Ting and Miksis [55] based on a Kirchhoff integral representation of the solution on a sphere, which results in a computationally expensive method. Extensions have been developed by many researchers $[2,25,28,29,31,33,34,35,38,46]$. In particular, Grote and Keller developed the exact nonreflecting boundary conditions for the three-dimensional time dependent wave equations based on spherical harmonics [28, 29]. They proved that the solution in the region $\Omega$ bounded by a sphere $\mathfrak{B}$ with exact boundary conditions is the same as the restriction to $\Omega$ of the solution of the original problem in the infinite region [28]. This successful method is then applied to Maxwell's equations [30] and elastic waves [31]. Another form of the exact nonreflecting boundary conditions for the scalar wave 
equation was obtained by Sofronov [54] independently. A systematic approach to the computation of exact conditions has been studied [1].

We have derived the exact nonreflecting boundary conditions for time-dependent poroelastic wave equations in three space dimensions. We enclose the scatter by spherical artificial boundary $\mathfrak{B}$. The choice of the sphere $\mathfrak{B}$ will facilitate the derivation of the boundary conditions. On $\mathfrak{B}$ we seek a boundary condition which ensures that the solution of the problem inside $\mathfrak{B}$ coincides with the solution of the original problem in the unbounded region.

The paper is organized as follows. In Section 2, we will present a decomposition of the displacement field in the poroelastic media into two compressional waves and a shear wave. Then in sections 3,4 , and 5 , we will derive the exact nonreflecting boundary conditions for the amplitude components of the coefficients appearing in the spherical harmonic expansion of the displacement field. We also show how to remove the high-order derivatives which occur in these exact boundary conditions. This makes the conditions more suitable for numerical computations. In Section 6 we derive three coupled systems of ordinary differential equations, which determine certain required auxiliary quantities in the exact boundary conditions. This makes the auxiliary quantities depending only on displacement solutions. In Section 7, we derive the final form of the exact nonreflecting boundary conditions for the displacement solutions in 3D poroelastic media. In Section 8, we present numerical results which demonstrate the effectiveness of our boundary conditions. Finally a conclusion is given.

\section{Decomposition of $3 \mathrm{D}$ poroelastic wave equations}

In this section, we will present a decomposition of the wave field which solves the $3 \mathrm{D}$ poroelastic wave equations. At low frequency, wave propagation in a 3D statistically poroelastic medium is described by Biot's equations [12]:

$$
\begin{gathered}
2 \sum_{j} \frac{\partial}{\partial x_{j}}\left(\mu \sigma_{i j}\right)+\frac{\partial}{\partial x_{i}}(\lambda \sigma-\alpha M \xi)=\frac{\partial^{2}}{\partial t^{2}}\left(\rho u_{i}+\rho_{f} w_{i}\right), \\
\frac{\partial}{\partial x_{i}}(\alpha M \sigma-M \xi)=\frac{\partial}{\partial t^{2}}\left(\rho_{f} u_{i}+\stackrel{\circ}{M} w_{i}\right)+\frac{\eta}{\kappa} \frac{\partial w_{i}}{\partial t},
\end{gathered}
$$

where $\stackrel{\circ}{M}=a \rho_{f} / \phi, \quad \xi=-\nabla \cdot \boldsymbol{w}, \quad M=\left(\frac{\phi}{K_{f}}+\frac{\alpha-\phi}{K_{s}}\right)^{-1}$, and $\alpha=1-\frac{K_{b}}{K_{s}}$. The physical meaning of the parameters appearing in (2.1)-(2.2) are presented as follows. $\mu$ is the shear modulus of the dry porous matrix, $\lambda$ is the Lamé constant of the saturated matrix, $\phi$ is the porosity, $\kappa$ is the permeability of the matrix, $\rho$ is the overall density of the saturated medium given by $\rho=\phi \rho_{f}+(1-\phi) \rho_{s}, \rho_{f}$ is the density of the pore fluid, $\rho_{s}$ is the density of the solid grains, $a$ is the tortuosity of the matrix, $K_{s}$ is the bulk modulus of the matrix material, $K_{f}$ is the bulk modulus of the pore fluid, and $K_{b}$ is the bulk modulus of the dry porous frame.

The system (2.1)-(2.2) has six equations. We choose the computational domain $\mathfrak{B}$ to be a sphere centered at the origin with radius $R$. Denote by $\mathfrak{B}^{\text {ext }}$ the region outside of $\mathfrak{B}$ and by $\mathfrak{B}^{\text {in }}$ the interior of $\mathfrak{B}$. In region $\mathfrak{B}^{\text {ext }}$, the medium is assumed to be linear, homogeneous, and isotropic. In addition, we assume that at $t=0$ the scattered field is confined to $\mathfrak{B}^{\text {in }}$.

In $\mathfrak{B}^{\text {ext }}$, the displacements $\boldsymbol{u}=\left(u_{1}, u_{2}, u_{3}\right)^{\mathrm{T}}$ and $\boldsymbol{w}=\left(w_{1}, w_{2}, w_{3}\right)^{\mathrm{T}}$ satisfy the poroelastic wave equations (2.1) and (2.2) with initial conditions

$$
\boldsymbol{u}=\boldsymbol{w}=0, \quad \frac{\partial \boldsymbol{u}}{\partial t}=\frac{\partial \boldsymbol{w}}{\partial t}=0, \quad t=0
$$


Rewriting (2.1) and (2.2) in vector form, we have

$$
\begin{aligned}
\frac{\partial^{2}}{\partial t^{2}}\left(\rho \boldsymbol{u}+\rho_{f} \boldsymbol{w}\right) & =\nabla((\lambda+\mu) \nabla \cdot \boldsymbol{u}+\alpha M \nabla \cdot \boldsymbol{w})+\nabla \cdot(\mu \nabla \boldsymbol{u}), \\
\frac{\partial^{2}}{\partial t^{2}}\left(\rho_{f} \boldsymbol{u}+\stackrel{\circ}{ } \boldsymbol{w}\right) & =\nabla(\alpha M \nabla \cdot \boldsymbol{u}+M \nabla \cdot \boldsymbol{w}) .
\end{aligned}
$$

In the above equations, we have neglected the viscous term by assuming that its influence is small. Taking curl in (2.4) and (2.5) and using the fact that the material is homogeneous in $\mathfrak{B}^{\text {ext }}$, we obtain

$$
\begin{aligned}
\frac{\partial^{2}}{\partial t^{2}}\left(\rho \omega+\rho_{f} \Omega\right) & =\nabla \times \nabla((\lambda+2 \mu) \nabla \cdot \boldsymbol{u}+\alpha M \nabla \cdot \boldsymbol{w})-\nabla \times \nabla \times(\mu \nabla \times \boldsymbol{u}) \\
& =-\mu \nabla \times \nabla \times \omega
\end{aligned}
$$

and

$$
\frac{\partial^{2}}{\partial t^{2}}\left(\rho_{f} \omega+\stackrel{\circ}{M} \Omega\right)=0,
$$

respectively, where we define $\operatorname{curl} \boldsymbol{u}:=\omega$ and $\operatorname{curl} \boldsymbol{w}:=\Omega$.

In the case with no viscosity, the rate of expansion of the solid is proportional to the rate of expansion of the fluid [12]. Therefore from (2.7) we get

$$
\Omega=-\frac{\rho_{f}}{\grave{M}} \omega
$$

Based on Helmholtz's theorem [32], we can decompose $\boldsymbol{u}$ and $\boldsymbol{w}$ into a field with vanishing curl and a field with vanishing divergence:

$$
\boldsymbol{u}=\nabla \psi_{1}+\boldsymbol{\Psi}_{1}, \quad \boldsymbol{w}=\nabla \psi_{2}+\boldsymbol{\Psi}_{2},
$$

where

$$
\nabla \cdot \Psi_{1}=0, \quad \nabla \cdot \Psi_{2}=0, \quad \nabla \times \psi_{1}=0, \quad \nabla \times \psi_{2}=0 .
$$

From the physical point of view, the irrotational fields $\nabla \times \psi_{1}$ and $\nabla \times \psi_{2}$ describe compressional waves while the solenoidal fields $\boldsymbol{\Psi}_{1}$ and $\boldsymbol{\Psi}_{2}$ describe shear waves.

The combination $(2.4) \times \stackrel{\circ}{M}-(2.5) \times \rho_{f}$ yields

$$
\begin{aligned}
\left(\stackrel{\circ}{M} \rho-\rho_{f}^{2}\right) \frac{\partial^{2} \boldsymbol{u}}{\partial t^{2}}=(\stackrel{\circ}{M} \lambda & \left.+2 \stackrel{\circ}{M} \mu-\alpha \rho_{f} M\right) \nabla \nabla \cdot \boldsymbol{u} \\
& +\left(\alpha \stackrel{\circ}{M} M-\rho_{f} M\right) \nabla \nabla \cdot \boldsymbol{w}-\stackrel{\circ}{M} \mu \nabla \times \nabla \times \boldsymbol{u},
\end{aligned}
$$

and $-(2.4) \times \rho_{f}+(2.5) \times \rho$ yields

$$
\begin{aligned}
\left(\stackrel{\circ}{M} \rho-\rho_{f}^{2}\right) \frac{\partial^{2} \boldsymbol{w}}{\partial t^{2}}=\left(\alpha \rho M-\rho_{f} \lambda-2 \rho_{f} \mu\right) \nabla \nabla \cdot \boldsymbol{u} \\
+\left(\rho M-\alpha \rho_{f} M\right) \nabla \nabla \cdot \boldsymbol{w}+\rho_{f} \mu \nabla \times \nabla \times \boldsymbol{u} .
\end{aligned}
$$

Substituting (2.9)-(2.10) into (2.11)-(2.12) and by using Lemma A.1 in Appendix A, we obtain

$$
\left(\stackrel{\circ}{M} \rho-\rho_{f}^{2}\right) \frac{\partial^{2} \psi_{1}}{\partial t^{2}}=\left(\stackrel{\circ}{M} \lambda+2 \stackrel{\circ}{M} \mu-\alpha \rho_{f} M\right) \nabla \cdot \nabla \psi_{1}+\left(\alpha \stackrel{\circ}{M} M-\rho_{f} M\right) \nabla \cdot \nabla \psi_{2},
$$




$$
\begin{gathered}
\left(\stackrel{\circ}{M} \rho-\rho_{f}^{2}\right) \frac{\partial^{2} \mathbf{\Psi}_{1}}{\partial t^{2}}=-\stackrel{\circ}{M} \mu\left(\nabla \times \nabla \times \mathbf{\Psi}_{1}\right), \\
\left(\stackrel{\circ}{M} \rho-\rho_{f}^{2}\right) \frac{\partial^{2} \psi_{2}}{\partial t^{2}}= \\
\left(\alpha \rho M-\rho_{f} \lambda-2 \rho_{f} \mu\right) \nabla \cdot \nabla \psi_{1}+\left(\rho M-\alpha \rho_{f} M\right) \nabla \cdot \nabla \psi_{2}, \\
\left(\stackrel{\circ}{M} \rho-\rho_{f}^{2}\right) \frac{\partial^{2} \mathbf{\Psi}_{2}}{\partial t^{2}}=\rho_{f} \mu\left(\nabla \times \nabla \times \mathbf{\Psi}_{1}\right) .
\end{gathered}
$$

Thus we have decomposed the elastic wavefields into the compressional waves satisfying (2.13) and (2.15) and the shear waves satisfying (2.14) and (2.16).

Let $r, \vartheta, \varphi$ be the polar coordinates and $\hat{\boldsymbol{r}}, \hat{\boldsymbol{\vartheta}}, \hat{\boldsymbol{\varphi}}$ be the corresponding unit vectors. We let $Y_{n m}$ be the $m n$-th spherical harmonic

$$
Y_{n m}(\vartheta, \phi)=\sqrt{\frac{(2 n+1)(n-|m|) !}{4 \pi(n+|m|) !}} P_{n}^{|m|}(\cos \vartheta) e^{i m \phi}, \quad n \geq 0,|m| \leq n,
$$

where $P_{n}^{|m|}$ is the associated Legendre function. By the orthogonality of $\left\{Y_{n m}\right\}$, the general solutions of (2.13) and (2.15) in $\mathfrak{B}^{\text {ext }}$ are

$$
\begin{aligned}
& \psi_{1}(r, \vartheta, \varphi, t)=\sum_{n \geq 0} \sum_{|m| \leq n} h_{n m}^{1}(r, t) Y_{n m}(\vartheta, \varphi), \quad r \geq R, \\
& \psi_{2}(r, \vartheta, \varphi, t)=\sum_{n \geq 0} \sum_{|m| \leq n} h_{n m}^{2}(r, t) Y_{n m}(\vartheta, \varphi), \quad r \geq R,
\end{aligned}
$$

where $h_{n m}^{1}(r, t)$ and $h_{n m}^{2}(r, t)$ are the Fourier coefficients. Inserting (2.18)-(2.19) into (2.13) and (2.15) respectively, we see that the Fourier coefficients satisfy

$$
\begin{aligned}
\left(\stackrel{\circ}{M} \rho-\rho_{f}^{2}\right) \frac{\partial^{2} h_{n m}^{1}}{\partial t^{2}}=(\stackrel{\circ}{M} & \left.\lambda+2 \stackrel{\circ}{M} \mu-\alpha \rho_{f} M\right)\left(\frac{\partial^{2}}{\partial r^{2}}+\frac{2}{r} \frac{\partial}{\partial r}-\frac{n(n+1)}{r^{2}}\right) h_{n m}^{1} \\
& +\left(\alpha \stackrel{\circ}{M} M-\rho_{f} M\right)\left(\frac{\partial^{2}}{\partial r^{2}}+\frac{2}{r} \frac{\partial}{\partial r}-\frac{n(n+1)}{r^{2}}\right) h_{n m}^{2}
\end{aligned}
$$

and

$$
\begin{aligned}
& \left(\stackrel{\circ}{M} \rho-\rho_{f}^{2}\right) \frac{\partial^{2} h_{n m}^{2}}{\partial t^{2}}=\left(\alpha \rho M-\rho_{f} \lambda-2 \rho_{f} \mu\right)\left(\frac{\partial^{2}}{\partial r^{2}}+\frac{2}{r} \frac{\partial}{\partial r}-\frac{n(n+1)}{r^{2}}\right) h_{n m}^{1} \\
& +\left(\rho M-\alpha \rho_{f} M\right)\left(\frac{\partial^{2}}{\partial r^{2}}+\frac{2}{r} \frac{\partial}{\partial r}-\frac{n(n+1)}{r^{2}}\right) h_{n m}^{2} .
\end{aligned}
$$

Next, we let $\boldsymbol{U}_{n m}$ and $\boldsymbol{V}_{n m}$ be the vector spherical harmonics [19]:

$$
\begin{gathered}
\boldsymbol{U}_{n m}(\vartheta, \phi)=\frac{r \nabla Y_{n m}}{\sqrt{n(n+1)}}=\frac{1}{\sqrt{n(n+1)}}\left[\frac{\partial Y_{n m}}{\partial \vartheta} \hat{\boldsymbol{\vartheta}}+\frac{1}{\sin \vartheta} \frac{\partial Y_{n m}}{\partial \phi} \hat{\boldsymbol{\phi}}\right], n \geq 1, \\
\boldsymbol{V}_{n m}(\vartheta, \phi)=\hat{\boldsymbol{r}} \times \boldsymbol{U}_{n m}=\frac{1}{\sqrt{n(n+1)}}\left[\frac{-1}{\sin \vartheta} \frac{\partial Y_{n m}}{\partial \phi} \hat{\boldsymbol{\vartheta}}+\frac{\partial Y_{n m}}{\partial \vartheta} \hat{\boldsymbol{\phi}}\right], n \geq 1
\end{gathered}
$$


Then the general solutions of (2.14) and (2.16) in $\mathfrak{B}^{\text {ext }}$ are

$$
\begin{aligned}
& \boldsymbol{\Psi}_{1}(r, \vartheta, \varphi, t)=\sum_{n \geq 1} \sum_{|m| \leq n}\left[f_{n m}^{1}(r, t) \boldsymbol{V}_{n m}+\nabla \times\left(g_{n m}^{1}(r, t) \boldsymbol{V}_{n m}\right)\right], \quad r \geq R, \\
& \boldsymbol{\Psi}_{2}(r, \vartheta, \varphi, t)=\sum_{n \geq 1} \sum_{|m| \leq n}\left[f_{n m}^{2}(r, t) \boldsymbol{V}_{n m}+\nabla \times\left(g_{n m}^{2}(r, t) \boldsymbol{V}_{n m}\right)\right], \quad r \geq R,
\end{aligned}
$$

where $f_{n m}^{1}(r, t), f_{n m}^{2}(r, t), g_{n m}^{1}(r, t)$, and $g_{n m}^{2}(r, t)$ are the Fourier coefficients. Inserting (2.24)-(2.25) into (2.14) and (2.16) respectively, we find that the Fourier coefficients satisfy the following four equations:

$$
\begin{aligned}
& \left(\stackrel{\circ}{M} \rho-\rho_{f}^{2}\right) \frac{\partial^{2} f_{n m}^{1}}{\partial t^{2}}=\stackrel{\circ}{M} \mu\left(\frac{\partial^{2} f_{n m}^{1}}{\partial r^{2}}+\frac{2}{r} \frac{\partial f_{n m}^{1}}{\partial r}-\frac{n(n+1)}{r^{2}} f_{n m}^{1}\right), \quad r \geq R, \\
& \left(\stackrel{\circ}{M} \rho-\rho_{f}^{2}\right) \frac{\partial^{2} g_{n m}^{1}}{\partial t^{2}}=\stackrel{\circ}{M} \mu\left(\frac{\partial^{2} g_{n m}^{1}}{\partial r^{2}}+\frac{2}{r} \frac{\partial g_{n m}^{1}}{\partial r}-\frac{n(n+1)}{r^{2}} g_{n m}^{1}\right), \quad r \geq R, \\
& \left(\stackrel{\circ}{M} \rho-\rho_{f}^{2}\right) \frac{\partial^{2} f_{n m}^{2}}{\partial t^{2}}=-\rho_{f} \mu\left(\frac{\partial^{2} f_{n m}^{1}}{\partial r^{2}}+\frac{2}{r} \frac{\partial f_{n m}^{1}}{\partial r}-\frac{n(n+1)}{r^{2}} f_{n m}^{1}\right), \quad r \geq R, \\
& \left(\stackrel{\circ}{M} \rho-\rho_{f}^{2}\right) \frac{\partial^{2} g_{n m}^{2}}{\partial t^{2}}=-\rho_{f} \mu\left(\frac{\partial^{2} g_{n m}^{1}}{\partial r^{2}}+\frac{2}{r} \frac{\partial g_{n m}^{1}}{\partial r}-\frac{n(n+1)}{r^{2}} g_{n m}^{1}\right), \quad r \geq R .
\end{aligned}
$$

Therefore, we have

$$
\rho_{f} \frac{\partial^{2} f_{n m}^{1}}{\partial t^{2}}+\stackrel{\circ}{M} \frac{\partial^{2} f_{n m}^{2}}{\partial t^{2}}=0, \quad \rho_{f} \frac{\partial^{2} g_{n m}^{1}}{\partial t^{2}}+\stackrel{\circ}{M} \frac{\partial^{2} g_{n m}^{2}}{\partial t^{2}}=0 .
$$

Next we further investigate the relations among the Fourier coefficients $f_{n m}^{1}, g_{n m}^{1}$, $h_{n m}^{1}, f_{n m}^{2}, g_{n m}^{2}$, and $h_{n m}^{2}$. Notice that we have

$$
\begin{aligned}
\Omega=\operatorname{curl} \boldsymbol{w}=\nabla & \times\left(\nabla \psi_{2}+\mathbf{\Psi}_{2}\right) \\
=\sum_{n \geq 1} \sum_{|m| \leq n}\{ & -\frac{\sqrt{n(n+1)} f_{n m}^{2}}{r} Y_{n m} \hat{\boldsymbol{r}} \\
& \left.\quad-\frac{1}{r} \frac{\partial\left(r f_{n m}^{2}\right)}{\partial r} \boldsymbol{U}_{n m}+\left[\frac{n(n+1) g_{n m}^{2}}{r^{2}}-\frac{1}{r} \frac{\partial^{2}\left(r g_{n m}^{2}\right)}{\partial r^{2}}\right] \boldsymbol{V}_{n m}\right\}
\end{aligned}
$$

and

$$
\begin{aligned}
\omega=\operatorname{curl} \boldsymbol{u}=\nabla & \times\left(\nabla \psi_{1}+\boldsymbol{\Psi}_{1}\right) \\
=\sum_{n \geq 1} \sum_{|m| \leq n}\{ & -\frac{\sqrt{n(n+1)} f_{n m}^{1}}{r} Y_{n m} \hat{\boldsymbol{r}} \\
& \left.\quad-\frac{1}{r} \frac{\partial\left(r f_{n m}^{1}\right)}{\partial r} \boldsymbol{U}_{n m}+\left[\frac{n(n+1) g_{n m}^{1}}{r^{2}}-\frac{1}{r} \frac{\partial^{2}\left(r g_{n m}^{1}\right)}{\partial r^{2}}\right] \boldsymbol{V}_{n m}\right\} .
\end{aligned}
$$

Using the relation between $\Omega$ and $\omega$ in (2.8), we have

$$
f_{n m}^{2}=-\frac{\rho_{f}}{M} f_{n m}^{1}, \quad g_{n m}^{2}=-\frac{\rho_{f}}{\grave{M}} g_{n m}^{1} .
$$


Thus $f_{n m}^{1}, g_{n m}^{1}, f_{n m}^{2}$, and $g_{n m}^{2}$ determine $\boldsymbol{\Psi}_{1}$ and $\boldsymbol{\Psi}_{2}$, i.e., the shear wave modes. Moreover, the proportional relation between $f_{n m}^{1}$ and $g_{n m}^{1}$ and the proportional relation between $f_{n m}^{2}$ and $g_{n m}^{2}$ show that only one shear wave mode is yielded.

Finally inserting (2.18)-(2.19) and (2.24)-(2.25) into (2.9), we obtain the displacement vectors:

$$
\begin{aligned}
\boldsymbol{u}(r, \vartheta, \varphi, t) & =\sum_{n \geq 0} \sum_{|m| \leq n} \boldsymbol{u}_{n m}(r, \vartheta, \varphi, t), \quad r \geq R, \\
\boldsymbol{w}(r, \vartheta, \varphi, t) & =\sum_{n \geq 0} \sum_{|m| \leq n} \boldsymbol{w}_{n m}(r, \vartheta, \varphi, t), \quad r \geq R
\end{aligned}
$$

where

$$
\begin{gathered}
\boldsymbol{u}_{n m}=f_{n m}^{1} \boldsymbol{V}_{n m}+\left(\frac{\sqrt{n(n+1)}}{r} h_{n m}^{1}-\frac{1}{r} \frac{\partial\left(r g_{n m}^{1}\right)}{\partial r}\right) \boldsymbol{U}_{n m} \\
+\left(\frac{\partial h_{n m}^{1}}{\partial r}-\frac{\sqrt{n(n+1)}}{r} g_{n m}^{1}\right) Y_{n m} \hat{\boldsymbol{r}}
\end{gathered}
$$

and

$$
\begin{gathered}
\boldsymbol{w}_{n m}=-\frac{\rho_{f}}{\stackrel{\circ}{M}} f_{n m}^{1} \boldsymbol{V}_{n m}+\left(\frac{\sqrt{n(n+1)}}{r} h_{n m}^{2}+\frac{\rho_{f}}{r \stackrel{\circ}{M}} \frac{\partial\left(r g_{n m}^{1}\right)}{\partial r}\right) \boldsymbol{U}_{n m} \\
+\left(\frac{\partial h_{n m}^{2}}{\partial r}+\frac{\sqrt{n(n+1)} \rho_{f}}{r \stackrel{\circ}{M}} g_{n m}^{1}\right) Y_{n m} \hat{\boldsymbol{r}} .
\end{gathered}
$$

For $n=0$, both $\boldsymbol{V}_{n m}$ and $\boldsymbol{U}_{n m}$ vanish. Equations (2.34)-(2.37) show that the exact solutions of $\boldsymbol{u}$ and $\boldsymbol{w}$ can be obtained by computing $f_{n m}^{1}, g_{n m}^{1}, h_{n m}^{1}, f_{n m}^{2}, g_{n m}^{2}$, and $h_{n m}^{2}$. Although $f_{n m}^{1}$ and $f_{n m}^{2}$ can be calculated by $\left(\boldsymbol{u}, \boldsymbol{V}_{n m}\right)$ and $\left(\boldsymbol{w}, \boldsymbol{V}_{n m}\right)$ respectively, $g_{n m}^{1}, h_{n m}^{1}, g_{n m}^{2}$, and $h_{n m}^{2}$ cannot be calculated by similar inner products.

\section{Exact boundary conditions for $\left(\boldsymbol{u}, \boldsymbol{V}_{n m}\right)$ and $\left(\boldsymbol{w}, \boldsymbol{V}_{n m}\right)$}

In this section, we derive exact boundary conditions for coefficients $f_{n m}^{1}(r, t)$ and $f_{n m}^{2}(r, t)$ by calculating the inner products $\left(\boldsymbol{u}, \boldsymbol{V}_{n m}\right)$ and $\left(\boldsymbol{w}, \boldsymbol{V}_{n m}\right), n \geq 1$. They are the exact nonreflecting boundary conditions for the modes of amplitude $f_{n m}^{1}$ and $f_{n m}^{2}$.

Following the analysis of Grote and Keller [28, 31], we introduce the integral operator $G_{n}[u]$ defined by

$$
G_{n}[u](r, t)= \begin{cases}r u(r, t), & n=0, \\ r \int_{\infty}^{r} \frac{\left(s^{2}-r^{2}\right)^{n-1} u(s, t)}{(2 s)^{n-1}(n-1) !} d s, & n \geq 1 .\end{cases}
$$

The operator $G_{n}[u]$ has the following four properties [31].

Lemma 3.1. Let $G_{n}[u](r, t)$ be defined by (3.1). Then

$$
r u(r, t)=\sum_{j=0} \frac{\gamma_{n j}}{r^{j}}\left(-\frac{\partial}{\partial r}\right)^{n-j} G_{n}[u](r, t),
$$

where

$$
\gamma_{n j}=\frac{(n+j) !}{(n-j) ! j ! 2^{j}}, \quad 0 \leq j \leq n
$$


Lemma 3.2. Let $G_{n}[u](r, t)$ be defined by (3.1). Then

$$
\frac{\partial}{\partial r}(r u(r, t))=-\sum_{j=0}^{n+1} \frac{\beta_{n j}}{r^{j}}\left(-\frac{\partial}{\partial r}\right)^{n+1-j} G_{n}[u](r, t),
$$

where

$$
\beta_{n j}= \begin{cases}1, & j=0 \\ \gamma_{n j}+(j-1) \gamma_{n, j-1}, & 1 \leq j \leq n, \\ n \gamma_{n n}, & j=n+1\end{cases}
$$

A direct consequence of lemmas 3.1 and 3.2 above is the following lemma.

Lemma 3.3. Let $G_{n}[u](r, t)$ be defined by (3.1), $\gamma_{n j}$ by (3.3), $\beta_{n j}$ by (3.5), and for all $n \geq 0$ set $\gamma_{n,-1}=0$. Then

$$
\frac{\partial}{\partial r}(u(r, t))=-\sum_{j=0}^{n+1} \frac{\beta_{n j}+\gamma_{n, j-1}}{r^{j+1}}\left(-\frac{\partial}{\partial r}\right)^{n+1-j} G_{n}[u](r, t) .
$$

The proof of lemmas 3.1, 3.2, and 3.3 can be found in [31]. Noting that $f_{n m}^{1}$ is proportional to $f_{n m}^{2}$, we only need to consider (2.26) or (2.28).

Lemma 3.4. If $f_{n m}^{1}$ satisfies (2.26), then

$$
\left(\stackrel{\circ}{M} \rho-\rho_{f}^{2}\right) \frac{\partial^{2} G_{n}\left[f_{n m}^{1}\right]}{\partial t^{2}}=\stackrel{\circ}{M} \mu \frac{\partial^{2} G_{n}\left[f_{n m}^{1}\right]}{\partial r^{2}} .
$$

The proof of this lemma can be found in [28].

As at $t=0, G_{n}\left[f_{n m}^{1}\right]$ and $\partial_{t} G_{n}\left[f_{n m}^{1}\right]$ vanish outside $\mathfrak{B}$. From (3.7) we obtain

$$
\left(\frac{\partial}{\partial t}+C_{S} \frac{\partial}{\partial r}\right) G_{n}\left[f_{n m}^{1}\right](r, t)=0
$$

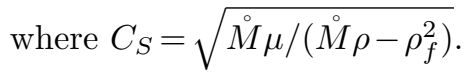

In order to derive a boundary condition for $f_{n m}^{1}$, we multiply (2.36) and (2.37) by $r$, take the inner product with $\boldsymbol{V}_{n m}$, and use Lemma 3.1 to obtain

$$
\begin{aligned}
& r f_{n m}^{1}(r, t)=\sum_{j=0}^{n} \frac{\gamma_{n j}}{r^{j}}\left(-\frac{\partial}{\partial r}\right)^{n-j} G_{n}\left[f_{n m}^{1}\right](r, t), \\
& r f_{n m}^{2}(r, t)=\sum_{j=0}^{n} \frac{\gamma_{n j}}{r^{j}}\left(-\frac{\partial}{\partial r}\right)^{n-j} G_{n}\left[f_{n m}^{2}\right](r, t) .
\end{aligned}
$$

Next we apply $\partial_{t}+C_{S} \partial_{r}$ to (3.9), and replace $-\partial_{r} G_{n}\left[f_{n m}^{1}\right]$ with $\partial_{t} G_{n}\left[f_{n m}^{1}\right] / C_{S}$ by using (3.8). This yields

$$
\left(\frac{\partial}{\partial t}+C_{S} \frac{\partial}{\partial r}\right)\left(r f_{n m}^{1}(r, t)\right)=-\frac{C_{S}}{r} \sum_{j=1}^{n} \frac{j \gamma_{n j}}{r^{j} C_{S}^{n-j}}\left(\frac{\partial}{\partial t}\right)^{n-j} G_{n}\left[f_{n m}^{1}\right](r, t), r=R .
$$

Equation (3.11) is an exact nonreflecting boundary condition for $f_{n m}^{1}(r, t)$ on $r=R$, but it involves time derivatives of $G_{n}\left[f_{n m}^{1}\right]$ up to order $n-1$. To compute the time 
derivatives of $G_{n}\left[f_{n m}^{1}\right]$ up to order $n-1$ for $r=R$, we again use (3.8) and (3.9) to substitute space derivatives with time derivatives. Thus, for $r=R$, we have

$$
\frac{1}{C_{S}^{n}} \frac{\partial^{n}}{\partial t^{n}} G_{n}\left[f_{n m}^{1}\right](r, t)=-\sum_{j=1}^{n} \frac{\gamma_{n j}}{r^{j} C_{S}^{n-j}}\left(\frac{\partial}{\partial t}\right)^{n-j} G_{n}\left[f_{n m}^{1}\right](r, t)+r f_{n m}^{1}(r, t),
$$

where we have used the fact $\gamma_{n 0}=1$ by definition. Equation (3.12) is the $n$ th-order ordinary differential equation for $G_{n}\left[f_{n m}^{1}\right](R, t)$.

To simplify the expression, we define the $n$-component vector function $\boldsymbol{\psi}_{n m}^{f^{1}}(t)$ by

$$
\psi_{n m}^{f^{1}, j}(t)=\frac{\gamma_{n j}}{R \gamma_{n 1} C_{S}^{n-j}}\left(\frac{\partial}{\partial t}\right)^{n-j} G_{n}\left[f_{n m}^{1}\right](R, t), \quad j=1, \cdots, n .
$$

In addition, we let $\boldsymbol{l}_{\boldsymbol{n}}=\left\{l_{n}^{j}\right\}$ be a constant $n$-component vector defined by

$$
l_{n}^{j}=\frac{n(n+1) j}{2 R^{j}}, \quad j=1, \cdots, n .
$$

With these new variables, the exact nonreflecting boundary condition (3.11) reduces to

$$
\left.\left(\frac{\partial}{\partial r}+\frac{1}{C_{S}} \frac{\partial}{\partial t}\right)\left(r \boldsymbol{u}, \boldsymbol{V}_{n m}\right)\right|_{r=R}=-\boldsymbol{l}_{n} \cdot \boldsymbol{\psi}_{n m}^{f^{1}}(t)
$$

Next, we note that by the definition of $\psi_{n m}^{f^{1}, j}$ we have

$$
\frac{1}{C_{S}} \frac{d}{d t} \psi_{n m}^{f^{1}, j}=\frac{\gamma_{n j}}{\gamma_{n, j-1}} \psi_{n m}^{f^{1}, j-1}=\frac{(n+j)(n+1-j)}{2 j} \psi_{n m}^{f^{1}, j-1}, \quad 2 \leq j \leq n .
$$

Since $f_{n m}^{1}$ and $\partial_{t} f_{n m}$ vanish identically for $r \geq R$ at $t=0$, so does $G_{n}\left[f_{n m}^{1}\right]$ and all its time derivatives up to order $n-1$. This implies that $\boldsymbol{\psi}_{n m}^{f^{1}}$ is equal to zero at $t=0$. Therefore we rewrite (3.12) and (3.16) as the following linear first-order ordinary differential equation:

$$
\frac{1}{C_{S}} \frac{d}{d t} \boldsymbol{\psi}_{n m}^{f^{1}}(t)=\boldsymbol{A}_{n} \boldsymbol{\psi}_{n m}^{f^{1}}+\left(\left.\boldsymbol{u}\right|_{r=R}, \boldsymbol{V}_{n m}\right) \boldsymbol{e}_{n}, \quad \boldsymbol{\psi}_{n m}^{f^{1}}(0)=0
$$

Here $\boldsymbol{e}_{n}=\left\{e_{n}^{j}\right\}=[1,0, \cdots, 0]^{T}$ is the constant unit vector with $n$ components, and $\boldsymbol{A}_{n}=\left\{A_{n}^{i j}\right\}$ is the constant $n \times n$ matrix defined by

$$
A_{n}^{i j}= \begin{cases}-n(n+1) /\left(2 R^{j}\right), & i=1 \\ (n+i)(n+1-i) /(2 i), & i=j+1 \\ 0, & \text { otherwise. }\end{cases}
$$

Since $\left(\boldsymbol{w}+\frac{\rho_{f}}{m} \boldsymbol{u}, \boldsymbol{V}_{n m}\right)=0$, from (3.15) we have

$$
\left.\left(\frac{\partial}{\partial r}+\frac{1}{C_{S}} \frac{\partial}{\partial t}\right)\left(r \boldsymbol{w}, \boldsymbol{V}_{n m}\right)\right|_{r=R}=\frac{\rho_{f}}{m} \boldsymbol{l}_{n} \cdot \boldsymbol{\psi}_{n m}^{f^{1}}(t)
$$


4. Exact boundary conditions for $\left(\boldsymbol{u}, \boldsymbol{U}_{n m}\right)$ and $\left(\boldsymbol{w}, \boldsymbol{U}_{n m}\right)$

In this section we will derive the exact boundary conditions for the components $\left(\boldsymbol{u}, \boldsymbol{U}_{n m}\right)$ and $\left(\boldsymbol{w}, \boldsymbol{U}_{n m}\right), n \geq 1$. To begin, we multiply (2.36) and (2.37) by $r$, take the inner product with $\boldsymbol{U}_{n m}$, and use lemmas 3.1 and 3.2 to obtain

$$
\begin{aligned}
\left(r \boldsymbol{u}, \boldsymbol{U}_{n m}\right)= & \sqrt{n(n+1)} h_{n m}^{1}-\frac{\partial}{\partial r}\left(r g_{n m}^{1}\right) \\
= & \sum_{j=0}^{n+1} \frac{\beta_{n j}}{r^{j}}\left(-\frac{\partial}{\partial r}\right)^{n+1-j} G_{n}\left[g_{n m}^{1}\right] \\
& +\sqrt{n(n+1)} \sum_{j=0}^{n} \frac{\gamma_{n j}}{r^{j+1}}\left(-\frac{\partial}{\partial r}\right)^{n-j} G_{n}\left[h_{n m}^{1}\right]
\end{aligned}
$$

and

$$
\begin{aligned}
\left(r \boldsymbol{w}, \boldsymbol{U}_{n m}\right)= & \sqrt{n(n+1)} h_{n m}^{2}-\frac{\partial}{\partial r}\left(r g_{n m}^{2}\right) \\
= & \sum_{j=0}^{n+1} \frac{\beta_{n j}}{r^{j}}\left(-\frac{\partial}{\partial r}\right)^{n+1-j} G_{n}\left[g_{n m}^{2}\right]+\sqrt{n(n+1)} \sum_{j=0}^{n} \frac{\gamma_{n j}}{r^{j+1}}\left(-\frac{\partial}{\partial r}\right)^{n-j} G_{n}\left[h_{n m}^{2}\right] \\
= & -\frac{\rho_{f}}{M} \sum_{j=0}^{n+1} \frac{\beta_{n j}}{r^{j}}\left(-\frac{\partial}{\partial r}\right)^{n+1-j} G_{n}\left[g_{n m}^{1}\right] \\
& +\sqrt{n(n+1)} \sum_{j=0}^{n} \frac{\gamma_{n j}}{r^{j+1}}\left(-\frac{\partial}{\partial r}\right)^{n-j} G_{n}\left[h_{n m}^{2}\right] .
\end{aligned}
$$

Using an argument similar to that used in Section 3 for $G_{n}\left[f_{n m}^{1}\right]$, we obtain

$$
\left(\frac{\partial}{\partial t}+C_{S} \frac{\partial}{\partial r}\right) G_{n}\left[g_{n m}^{1}\right](r, t)=0
$$

Applying the operator $\partial_{t}+C_{S} \partial_{r}$ to $\left(r \boldsymbol{u}, \boldsymbol{U}_{n m}\right)$ and $\left(r \boldsymbol{w}, \boldsymbol{U}_{n m}\right)$, and replacing the space derivative $-\partial_{r} G_{n}\left[g_{n m}^{1}\right]$ with the time derivative $\partial_{t} G_{n}\left[g_{n m}^{1}\right] / C_{S}$, we obtain

$$
\begin{aligned}
\left(\frac{\partial}{\partial t}+C_{S} \frac{\partial}{\partial r}\right)\left(r \boldsymbol{u}, \boldsymbol{U}_{n m}\right)=- & C_{S} \sqrt{n(n+1)} \sum_{j=1}^{n+1} \frac{j \gamma_{n j-1}}{r^{j+1}}\left(-\frac{\partial}{\partial r}\right)^{n+1-j} G_{n}\left[h_{n m}^{1}\right] \\
& +\sqrt{n(n+1)} \sum_{j=0}^{n} \frac{\gamma_{n j}}{r^{j+1}}\left(-\frac{\partial}{\partial r}\right)^{n-j}\left(\frac{\partial}{\partial t}+C_{S} \frac{\partial}{\partial r}\right) G_{n}\left[h_{n m}^{1}\right] \\
& -C_{S} \sum_{j=1}^{n+1} \frac{j \beta_{n j}}{r^{j+1}}\left(\frac{1}{C_{S}} \frac{\partial}{\partial t}\right)^{n+1-j} G_{n}\left[g_{n m}^{1}\right]
\end{aligned}
$$

and

$$
\begin{aligned}
\left(\frac{\partial}{\partial t}+C_{S} \frac{\partial}{\partial r}\right)\left(r \boldsymbol{w}, \boldsymbol{U}_{n m}\right)=- & C_{S} \sqrt{n(n+1)} \sum_{j=1}^{n+1} \frac{j \gamma_{n j-1}}{r^{j+1}}\left(-\frac{\partial}{\partial r}\right)^{n+1-j} G_{n}\left[h_{n m}^{2}\right] \\
& +\sqrt{n(n+1)} \sum_{j=0}^{n} \frac{\gamma_{n j}}{r^{j+1}}\left(-\frac{\partial}{\partial r}\right)^{n-j}\left(\frac{\partial}{\partial t}+C_{S} \frac{\partial}{\partial r}\right) G_{n}\left[h_{n m}^{2}\right]
\end{aligned}
$$




$$
+\frac{C_{S} \rho_{f}}{M} \sum_{j=1}^{n+1} \frac{j \beta_{n j}}{r^{j+1}}\left(\frac{1}{C_{S}} \frac{\partial}{\partial t}\right)^{n+1-j} G_{n}\left[g_{n m}^{1}\right] .
$$

By using the operator $\partial_{t}+C_{S} \partial_{r}$, we have annihilated the $(n+1)$ st derivatives of $G_{n}\left[g_{n m}^{1}\right]$, but we have introduced the $(n+1)$ st derivatives of $G_{n}\left[h_{n m}^{1}\right]$ and $G_{n}\left[h_{n m}^{2}\right]$, i.e., $\left(-\partial_{r}\right)^{n}\left(\partial_{t}+C_{S} \partial_{r}\right) G_{n}\left[h_{n m}^{1}\right]$ and $\left(-\partial_{r}\right)^{n}\left(\partial_{t}+C_{S} \partial_{r}\right) G_{n}\left[h_{n m}^{2}\right]$.

Since in (4.4) and (4.5), the $(n+1)$ st derivatives of $G_{n}\left[h_{n m}^{1}\right]$ and $G_{n}\left[h_{n m}^{2}\right]$ involve both time and space derivatives, we cannot substitute space derivatives with time derivatives directly. We now seek an alternative. To do so, we first solve $\left(\partial_{r}\right)^{s} G_{n}\left[h_{n m}^{1}\right]$ and $\left(\partial_{r}\right)^{s} G_{n}\left[h_{n m}^{2}\right]$ to get a relation between time and space derivatives. Then we substitute the resulting expression into (4.4) and (4.5) to make the $(n+1)$ st derivatives only involve time derivatives.

Lemma 4.1. Let $h_{n m}^{1}$ and $h_{n m}^{2}$ satisfy (2.20) and (2.21). Then

$$
\begin{aligned}
\left(\stackrel{\circ}{M} \rho-\rho_{f}^{2}\right) \frac{\partial^{2} G_{n}\left[h_{n m}^{1}\right]}{\partial t^{2}}=(\stackrel{\circ}{M} & \left.+2 \stackrel{\circ}{M} \mu-\alpha \rho_{f} M\right) \frac{\partial^{2} G_{n}\left[h_{n m}^{1}\right]}{\partial r^{2}} \\
& +\left(\alpha \stackrel{\circ}{M} M-\rho_{f} M\right) \frac{\partial^{2} G_{n}\left[h_{n m}^{2}\right]}{\partial r^{2}}
\end{aligned}
$$

and

$$
\begin{array}{r}
\left(\stackrel{\circ}{M} \rho-\rho_{f}^{2}\right) \frac{\partial^{2} G_{n}\left[h_{n m}^{2}\right]}{\partial t^{2}}=\left(\alpha \rho M-\rho_{f} \lambda-2 \rho_{f} \mu\right) \frac{\partial^{2} G_{n}\left[h_{n m}^{1}\right]}{\partial r^{2}} \\
+\left(\rho M-\alpha \rho_{f} M\right) \frac{\partial^{2} G_{n}\left[h_{n m}^{2}\right]}{\partial r^{2}} .
\end{array}
$$

The proof of Lemma 4.1 is given in Appendix B.

Denoting $\boldsymbol{H}_{n}=\left(G_{n}\left[h_{n m}^{1}\right], G_{n}\left[h_{n m}^{2}\right]\right)^{\mathrm{T}}$, we rewrite (4.6) and (4.7) as

$$
\begin{aligned}
& \left(\begin{array}{cc}
\stackrel{\circ}{M} \rho-\rho_{f}^{2} & 0 \\
0 & \stackrel{\circ}{M} \rho-\rho_{f}^{2}
\end{array}\right) \frac{\partial^{2} \boldsymbol{H}_{n}}{\partial t^{2}} \\
& =\left(\begin{array}{cc}
\stackrel{\circ}{M} \lambda+2 \stackrel{\circ}{M} \mu-\alpha \rho_{f} M & \alpha \stackrel{\circ}{M} M-\rho_{f} M \\
\alpha \rho M-\rho_{f} \lambda-2 \rho_{f} \mu & \rho M-\alpha \rho_{f} M
\end{array}\right) \frac{\partial^{2} \boldsymbol{H}_{n}}{\partial r^{2}} .
\end{aligned}
$$

For simplicity, we denote

$$
\boldsymbol{A}=\left(\begin{array}{cc}
\stackrel{\circ}{M} \lambda+2 \stackrel{\circ}{M} \mu-\alpha \rho_{f} M & \alpha \stackrel{\circ}{M} M-\rho_{f} M \\
\alpha \rho M-\rho_{f} \lambda-2 \rho_{f} \mu & \rho M-\alpha \rho_{f} M
\end{array}\right),
$$

and let $\lambda_{1}$ and $\lambda_{2}$ be the eigenvalues of $\boldsymbol{A}$. For practical problems both $\lambda_{1}$ and $\lambda_{2}$ are positive. If $\lambda_{1} \neq \lambda_{2}$, without loss of generality we assume $\lambda_{1}>\lambda_{2}$, and then there exists an invertible matrix $\boldsymbol{T}_{1}$ satisfying $\boldsymbol{A}=\boldsymbol{T}_{1}\left(\begin{array}{cc}\lambda_{1} & 0 \\ 0 & \lambda_{2}\end{array}\right) \boldsymbol{T}_{1}^{-1}$. Thus

$$
\left(\stackrel{\circ}{M} \rho-\rho_{f}^{2}\right) \frac{\partial^{2}\left(\boldsymbol{T}_{1}^{-1} \boldsymbol{H}_{n}\right)}{\partial t^{2}}=\left(\begin{array}{cc}
\lambda_{1} & 0 \\
0 & \lambda_{2}
\end{array}\right) \frac{\partial^{2}\left(\boldsymbol{T}_{1}^{-1} \boldsymbol{H}_{n}\right)}{\partial r^{2}} .
$$

Denoting $\tilde{\boldsymbol{H}}_{n}=\left(\tilde{\boldsymbol{H}}_{n}^{1}, \tilde{\boldsymbol{H}}_{n}^{2}\right)^{\mathrm{T}}=\boldsymbol{T}_{1}^{-1} \boldsymbol{H}_{n}$, from (4.10) we have

$$
\left(\stackrel{\circ}{M} \rho-\rho_{f}^{2}\right) \frac{\partial^{2} \tilde{\boldsymbol{H}}_{n}^{1}}{\partial t^{2}}=\lambda_{1} \frac{\partial^{2} \tilde{\boldsymbol{H}}_{n}^{1}}{\partial r^{2}}, \quad\left(\stackrel{\circ}{M} \rho-\rho_{f}^{2}\right) \frac{\partial^{2} \tilde{\boldsymbol{H}}_{n}^{2}}{\partial t^{2}}=\lambda_{2} \frac{\partial^{2} \tilde{\boldsymbol{H}}_{n}^{2}}{\partial r^{2}} .
$$


From (4.11), we obtain

$$
\left(\frac{\partial}{\partial t}+C_{P 1} \frac{\partial}{\partial r}\right) \tilde{\boldsymbol{H}}_{n}^{1}=0, \quad\left(\frac{\partial}{\partial t}+C_{P 2} \frac{\partial}{\partial r}\right) \tilde{\boldsymbol{H}}_{n}^{2}=0
$$

where $C_{P 1}=\sqrt{\lambda_{1} /\left(\stackrel{\circ}{M} \rho-\rho_{f}^{2}\right)}, \quad C_{P 2}=\sqrt{\lambda_{2} /\left(\stackrel{\circ}{M} \rho-\rho_{f}^{2}\right)} . \quad$ Letting $\boldsymbol{T}_{1}^{-1}=\left\{t_{i j}\right\}, \quad(i, j=$ $1,2),(4.12)$ can be written as

$$
\begin{aligned}
& \left(\frac{\partial}{\partial t}+C_{P 1} \frac{\partial}{\partial r}\right)\left(t_{11} G_{n}\left[h_{n m}^{1}\right]+t_{12} G_{n}\left[h_{n m}^{2}\right]\right)=0, \\
& \left(\frac{\partial}{\partial t}+C_{P 2} \frac{\partial}{\partial r}\right)\left(t_{21} G_{n}\left[h_{n m}^{1}\right]+t_{22} G_{n}\left[h_{n m}^{2}\right]\right)=0 .
\end{aligned}
$$

Next, we derive a relation between the time and space derivatives of $G_{n}\left[h_{n m}^{1}\right]$ and $G_{n}\left[h_{n m}^{2}\right]$. We rewrite (4.13)-(4.14) as

$$
C_{P 1} t_{11} \frac{\partial}{\partial r} G_{n}\left[h_{n m}^{1}\right]+C_{P 1} t_{12} \frac{\partial}{\partial r} G_{n}\left[h_{n m}^{2}\right]=-\frac{\partial}{\partial t}\left(t_{11} G_{n}\left[h_{n m}^{1}\right]+t_{12} G_{n}\left[h_{n m}^{2}\right]\right)
$$

and

$$
C_{P 2} t_{21} \frac{\partial}{\partial r} G_{n}\left[h_{n m}^{1}\right]+C_{P 2} t_{22} \frac{\partial}{\partial r} G_{n}\left[h_{n m}^{2}\right]=-\frac{\partial}{\partial t}\left(t_{21} G_{n}\left[h_{n m}^{1}\right]+t_{22} G_{n}\left[h_{n m}^{2}\right]\right) .
$$

Solving for $\partial_{r} G_{n}\left[h_{n m}^{1}\right]$ from (4.15) and $\partial_{r} G_{n}\left[h_{n m}^{2}\right]$ from (4.16), respectively, yields

$$
\begin{gathered}
\frac{\partial}{\partial r} G_{n}\left[h_{n m}^{1}\right]=\frac{1}{A} \frac{\partial}{\partial t}\left(B G_{n}\left[h_{n m}^{1}\right]+C G_{n}\left[h_{n m}^{2}\right]\right), \\
\frac{\partial}{\partial r} G_{n}\left[h_{n m}^{2}\right]=-\frac{1}{A} \frac{\partial}{\partial t}\left(D G_{n}\left[h_{n m}^{1}\right]+E G_{n}\left[h_{n m}^{2}\right]\right),
\end{gathered}
$$

where

$$
\begin{aligned}
& A=C_{P 1} C_{P 2}\left(t_{11} t_{22}-t_{12} t_{21}\right), \\
& B=C_{P 1} t_{12} t_{21}-C_{P 2} t_{11} t_{22}, \\
& C=\left(C_{P 1}-C_{P 2}\right) t_{12} t_{22}, \\
& D=\left(C_{P 1}-C_{P 2}\right) t_{11} t_{21}, \\
& E=C_{P 1} t_{11} t_{22}-C_{P 2} t_{12} t_{21} .
\end{aligned}
$$

Now we calculate $\partial_{r}^{s} G_{n}\left[h_{n m}^{1}\right]$ and $\partial_{r}^{s} G_{n}\left[h_{n m}^{2}\right](s \geq 1)$. Let

$$
\begin{gathered}
\left(\frac{\partial}{\partial r}\right)^{s} G_{n}\left[h_{n m}^{1}\right]=\frac{1}{A^{s}}\left(\frac{\partial}{\partial t}\right)^{s}\left(a_{s} G_{n}\left[h_{n m}^{1}\right]+b_{s} G_{n}\left[h_{n m}^{2}\right]\right), \\
\left(\frac{\partial}{\partial r}\right)^{s} G_{n}\left[h_{n m}^{2}\right]=\frac{1}{(-A)^{s}}\left(\frac{\partial}{\partial t}\right)^{s}\left(c_{s} G_{n}\left[h_{n m}^{1}\right]+d_{s} G_{n}\left[h_{n m}^{2}\right]\right) .
\end{gathered}
$$

Obviously, for $s=1$,

$$
a_{1}=B, \quad b_{1}=C, \quad C_{P 1}=D, \quad d_{1}=E,
$$


so that (4.20) and (4.21) hold. For the general case, since

$$
\begin{aligned}
& \left(\frac{\partial}{\partial r}\right)^{s+1} G_{n}\left[h_{n m}^{1}\right] \\
= & \frac{1}{A^{s}}\left\{a_{s}\left(\frac{\partial}{\partial t}\right)^{s} \frac{\partial}{\partial r} G_{n}\left[h_{n m}^{1}\right]+b_{s}\left(\frac{\partial}{\partial t}\right)^{s} \frac{\partial}{\partial r} G_{n}\left[h_{n m}^{2}\right]\right\} \\
= & \frac{1}{A^{s+1}}\left\{\left(\frac{\partial}{\partial t}\right)^{s+1}\left(a_{s} B-b_{s} D\right) G_{n}\left[h_{n m}^{1}\right]+\left(\frac{\partial}{\partial t}\right)^{s+1}\left(a_{s} C-b_{s} E\right) G_{n}\left[h_{n m}^{2}\right]\right\} \\
= & \frac{1}{A^{s+1}}\left(\frac{\partial}{\partial t}\right)^{s+1}\left\{a_{s+1} G_{n}\left[h_{n m}^{1}\right]+b_{s+1} G_{n}\left[h_{n m}^{2}\right]\right\},
\end{aligned}
$$

we conclude that

$$
a_{s+1}=a_{s} B-b_{s} D, \quad b_{s+1}=a_{s} C-b_{s} E .
$$

Rewriting (4.23) in matrix form

$$
\left(\begin{array}{l}
a_{s+1} \\
b_{s+1}
\end{array}\right)=\left(\begin{array}{c}
B-D \\
C-E
\end{array}\right)\left(\begin{array}{l}
a_{s} \\
b_{s}
\end{array}\right)=\left(\begin{array}{l}
B-D \\
C-E
\end{array}\right)^{s}\left(\begin{array}{l}
a_{1} \\
b_{1}
\end{array}\right),
$$

and denoting by $\tilde{\lambda}_{1}$ and $\tilde{\lambda}_{2}$ the eigenvalues of the matrix $\left(\begin{array}{l}B-D \\ C-E\end{array}\right)$, we see that $\tilde{\lambda}_{1}=C_{P 1}\left(t_{12} t_{21}-t_{11} t_{22}\right)$ and $\tilde{\lambda}_{2}=C_{P 2}\left(t_{12} t_{21}-t_{11} t_{22}\right)$. Let $\left(t_{21}, t_{22}\right)^{T}$ and $\left(t_{11}, t_{12}\right)^{T}$ be the eigenvectors corresponding to $\tilde{\lambda}_{1}$ and $\tilde{\lambda}_{2}$ respectively. Then

$$
\begin{aligned}
\left(\begin{array}{l}
B-D \\
C-E
\end{array}\right)^{s} & =\left(\begin{array}{cc}
t_{11} & t_{21} \\
t_{12} & t_{22}
\end{array}\right)\left(\begin{array}{cc}
\tilde{\lambda}_{2}^{s} & 0 \\
0 & \tilde{\lambda}_{1}^{s}
\end{array}\right)\left(\begin{array}{cc}
t_{11} & t_{21} \\
t_{12} & t_{22}
\end{array}\right)^{-1} \\
& =\frac{1}{\left(t_{11} t_{22}-t_{12} t_{21}\right)}\left(\begin{array}{l}
-t_{12} t_{21} \tilde{\lambda}_{1}^{s}+t_{11} t_{22} \tilde{\lambda}_{2}^{s} t_{11} t_{21} \tilde{\lambda}_{1}^{s}-t_{11} t_{21} \tilde{\lambda}_{2}^{s} \\
-t_{12} t_{22} \tilde{\lambda}_{1}^{s}+t_{12} t_{22} \tilde{\lambda}_{2}^{s} t_{11} t_{22} \tilde{\lambda}_{1}^{s}-t_{12} t_{21} \tilde{\lambda}_{2}^{s}
\end{array}\right) .
\end{aligned}
$$

Inserting (4.25) into (4.24), we obtain

$$
\left\{\begin{array}{l}
a_{s}=(-1)^{s-1}\left(C_{P 1}^{s} t_{12} t_{21}-C_{P 2}^{s} t_{11} t_{22}\right)\left(t_{11} t_{22}-t_{12} t_{21}\right)^{s-1}, \\
b_{s}=(-1)^{s-1}\left(C_{P 1}^{s}-C_{P 2}^{s}\right) t_{12} t_{22}\left(t_{11} t_{22}-t_{12} t_{21}\right)^{s-1} .
\end{array}\right.
$$

Using a similar procedure for (4.21), we get

$$
\left\{\begin{array}{l}
c_{s}=\left(C_{P 1}^{s}-C_{P 2}^{s}\right) t_{11} t_{21}\left(t_{11} t_{22}-t_{12} t_{21}\right)^{s-1}, \\
d_{s}=\left(C_{P 1}^{s} t_{11} t_{22}-C_{P 2}^{s} t_{12} t_{21}\right)\left(t_{11} t_{22}-t_{12} t_{21}\right)^{s-1} .
\end{array}\right.
$$

Thus we have expressed the space derivatives of $G_{n}\left[h_{n m}^{1}\right]$ and $G_{n}\left[h_{n m}^{2}\right]$ in terms of time derivatives. Substituting (4.20)-(4.21) into (4.4)-(4.5), we obtain

$$
\begin{aligned}
&\left(\frac{\partial}{\partial t}+C_{S} \frac{\partial}{\partial r}\right)\left(r \boldsymbol{u}, \boldsymbol{U}_{n m}\right) \\
&=-C_{S} \sum_{j=1}^{n+1} \frac{j \beta_{n j}}{r^{j+1} C_{S}^{n+1-j}}\left(\frac{\partial}{\partial t}\right)^{n+1-j} G_{n}\left[g_{n m}^{1}\right]+\sqrt{n(n+1)} \\
& \quad \times \sum_{j=0}^{n+1}\left\{\frac{(-1)^{n-j} \gamma_{n j}}{r^{j+1} A^{n-j}}\left(\frac{\partial}{\partial t}\right)^{n+1-j}\left(a_{n-j} G_{n}\left[h_{n m}^{1}\right]+b_{n-j} G_{n}\left[h_{n m}^{2}\right]\right)\right.
\end{aligned}
$$




$$
\begin{gathered}
\left.+\frac{(-1)^{n-j} C_{S} \gamma_{n j}}{r^{j+1} A^{n+1-j}}\left(\frac{\partial}{\partial t}\right)^{n+1-j}\left(a_{n+1-j} G_{n}\left[h_{n m}^{1}\right]+b_{n+1-j} G_{n}\left[h_{n m}^{2}\right]\right)\right\} \\
+C_{S} \sqrt{n(n+1)} \\
\times\left\{\sum_{j=1}^{n+1} \frac{(-1)^{n-j} j \gamma_{n, j-1}}{r^{j+1} A^{n+1-j}}\left(\frac{\partial}{\partial t}\right)^{n+1-j}\left(a_{n+1-j} G_{n}\left[h_{n m}^{1}\right]+b_{n+1-j} G_{n}\left[h_{n m}^{2}\right]\right)\right\}
\end{gathered}
$$

and

$$
\begin{aligned}
&\left(\frac{\partial}{\partial t}+\right.\left.C_{S} \frac{\partial}{\partial r}\right)\left(r \boldsymbol{w}, \boldsymbol{U}_{n m}\right) \\
&=C_{S} \frac{\rho_{f}}{m} \sum_{j=1}^{n+1} \frac{j \beta_{n j}}{r^{j+1} C_{S}^{n+1-j}}\left(\frac{\partial}{\partial t}\right)^{n+1-j} G_{n}\left[g_{n m}^{1}\right]+\sqrt{n(n+1)} \\
& \quad \times \sum_{j=0}^{n+1}\left\{\frac{\gamma_{n j}}{r^{j+1} A^{n-j}}\left(\frac{\partial}{\partial t}\right)^{n+1-j}\left(c_{n-j} G_{n}\left[h_{n m}^{1}\right]+d_{n-j} G_{n}\left[h_{n m}^{2}\right]\right)\right. \\
&\left.\quad-\frac{C_{S} \gamma_{n j}}{r^{j+1} A^{n+1-j}}\left(\frac{\partial}{\partial t}\right)^{n+1-j}\left(c_{n+1-j} G_{n}\left[h_{n m}^{1}\right]+d_{n+1-j} G_{n}\left[h_{n m}^{2}\right]\right)\right\} \\
&-C_{S} \sqrt{n(n+1)} \\
& \quad \times\left\{\sum_{j=1}^{n+1} \frac{j \gamma_{n, j-1}}{r^{j+1} A^{n+1-j}}\left(\frac{\partial}{\partial t}\right)^{n+1-j}\left(c_{n+1-j} G_{n}\left[h_{n m}^{1}\right]+d_{n+1-j} G_{n}\left[h_{n m}^{2}\right]\right)\right\}
\end{aligned}
$$

The $(n+1)$ st derivatives of $G_{n}\left[h_{n m}^{1}\right]$ and $G_{n}\left[h_{n m}^{2}\right]$ in (4.28) and (4.29) only involve time derivatives. We now seek a partial differential operator to annihilate these highorder time derivatives. From Grote and Keller [31], the operator is

$$
\begin{aligned}
& r \hat{\boldsymbol{r}} \times \nabla \times\left(\left(\hat{\boldsymbol{r}} \cdot \boldsymbol{u}_{n m}\right) \hat{\boldsymbol{r}}\right)=\left[\sqrt{n(n+1)} \frac{\partial}{\partial r} h_{n m}^{1}-n(n+1) \frac{g_{n m}^{1}}{r}\right] \boldsymbol{U}_{n m}, \\
& r \hat{\boldsymbol{r}} \times \nabla \times\left(\left(\hat{\boldsymbol{r}} \cdot \boldsymbol{w}_{n m}\right) \hat{\boldsymbol{r}}\right)=\left[\sqrt{n(n+1)} \frac{\partial}{\partial r} h_{n m}^{2}-n(n+1) \frac{g_{n m}^{2}}{r}\right] \boldsymbol{U}_{n m} .
\end{aligned}
$$

Taking the inner product of (4.30) and (4.31) with $\boldsymbol{U}_{n m}$, and by using the lemmas 3.1 and 3.3 , we obtain

$$
\begin{aligned}
(r \hat{\boldsymbol{r}} \times & \left.\nabla \times(\hat{\boldsymbol{r}} \cdot \boldsymbol{u}) \hat{\boldsymbol{r}}, \boldsymbol{U}_{n m}\right) \\
=-\sqrt{n(n+1)} \sum_{j=0}^{n+1} \frac{(-1)^{n+1-j}\left(\beta_{n j}+\gamma_{n, j-1}\right)}{r^{j+1} A^{n+1-j}} & \times\left(\frac{\partial}{\partial t}\right)^{n+1-j}\left(a_{n+1-j} G_{n}\left[h_{n m}^{1}\right]+b_{n+1-j} G_{n}\left[h_{n m}^{2}\right]\right) \\
- & n(n+1) \sum_{j=1}^{n+1} \frac{\gamma_{n, j-1}}{r^{j+1} C_{S}^{n+1-j}}\left(\frac{\partial}{\partial t}\right)^{n+1-j} G_{n}\left[g_{n m}^{1}\right]
\end{aligned}
$$

and

$$
\left(r \hat{\boldsymbol{r}} \times \nabla \times((\hat{\boldsymbol{r}} \cdot \boldsymbol{w}) \hat{\boldsymbol{r}}), \boldsymbol{U}_{n m}\right)
$$




$$
\begin{aligned}
=-\sqrt{n(n+1)} & \sum_{j=0}^{n+1} \frac{\left(\beta_{n j}+\gamma_{n, j-1}\right)}{r^{j+1} A^{n+1-j}} \\
& \times\left(\frac{\partial}{\partial t}\right)^{n+1-j}\left(c_{n+1-j} G_{n}\left[h_{n m}^{1}\right]+d_{n+1-j} G_{n}\left[h_{n m}^{2}\right]\right) \\
+ & \frac{n(n+1) \rho_{f}}{m} \sum_{j=1}^{n+1} \frac{\gamma_{n, j-1}}{r^{j+1} C_{S}^{n+1-j}}\left(\frac{\partial}{\partial t}\right)^{n+1-j} G_{n}\left[g_{n m}^{1}\right] .
\end{aligned}
$$

Through the use of suitable linear combinations of (4.28), (4.29), (4.32), and (4.33), we can annihilate the $(n+1)$ st derivatives in (4.32) and (4.33). This is illustrated in the following theorem.

THEOREM 4.2. There exist constants $y_{1}, z_{1}, y_{2}$, and $z_{2}$ which are independent of $n$ such that the $(n+1)$ st derivatives in

$$
\left(\frac{\partial}{\partial t}+C_{S} \frac{\partial}{\partial r}\right)\left(r \boldsymbol{u}, \boldsymbol{U}_{n m}\right)+y_{1}\left(r \hat{\boldsymbol{r}} \times \nabla \times(\hat{\boldsymbol{r}} \cdot \boldsymbol{u}) \hat{\boldsymbol{r}}, \boldsymbol{U}_{n m}\right)+z_{1}\left(r \hat{\boldsymbol{r}} \times \nabla \times(\hat{\boldsymbol{r}} \cdot \boldsymbol{w}) \hat{\boldsymbol{r}}, \boldsymbol{U}_{n m}\right)
$$

and

$$
\left(\frac{\partial}{\partial t}+C_{S} \frac{\partial}{\partial r}\right)\left(r \boldsymbol{w}, \boldsymbol{U}_{n m}\right)+y_{2}\left(r \hat{\boldsymbol{r}} \times \nabla \times(\hat{\boldsymbol{r}} \cdot \boldsymbol{u}) \hat{\boldsymbol{r}}, \boldsymbol{U}_{n m}\right)+z_{2}\left(r \hat{\boldsymbol{r}} \times \nabla \times(\hat{\boldsymbol{r}} \cdot \boldsymbol{w}) \hat{\boldsymbol{r}}, \boldsymbol{U}_{n m}\right)
$$

are cancelled, i.e., the coefficients of $\partial_{t}^{n+1} G_{n}\left[h_{n m}^{1}\right]$ and $\partial_{t}^{n+1} G_{n}\left[h_{n m}^{2}\right]$ vanish.

Proof. We will only give the proof for (4.34). The proof of (4.35) is similar. To make the $(n+1)$ st derivatives in (4.34) vanish, we need

$$
\begin{gathered}
\frac{(-1)^{n}}{r A^{n}}\left(\frac{\partial}{\partial t}\right)^{n+1}\left(a_{n} G_{n}\left[h_{n m}^{1}\right]+b_{n} G_{n}\left[h_{n m}^{2}\right]\right) \\
+\frac{(-1)^{n} C_{S}}{r A^{n+1}}\left(\frac{\partial}{\partial t}\right)^{n+1}\left(a_{n+1} G_{n}\left[h_{n m}^{1}\right]+b_{n+1} G_{n}\left[h_{n m}^{2}\right]\right) \\
-y_{1} \frac{(-1)^{n+1}}{r A^{n+1}}\left(\frac{\partial}{\partial t}\right)^{n+1}\left(a_{n+1} G_{n}\left[h_{n m}^{1}\right]+b_{n+1} G_{n}\left[h_{n m}^{2}\right]\right) \\
-z_{1} \frac{1}{r A^{n+1}}\left(\frac{\partial}{\partial t}\right)^{n+1}\left(c_{n+1} G_{n}\left[h_{n m}^{1}\right]+d_{n+1} G_{n}\left[h_{n m}^{2}\right]\right)=0 .
\end{gathered}
$$

Thus the coefficients of $\partial_{t}^{n+1} G_{n}\left[h_{n m}^{1}\right]$ and $\partial_{t}^{n+1} G_{n}\left[h_{n m}^{2}\right]$ vanish. Solving the resulting system for $y_{1}$ and $z_{1}$ yields

$$
y_{1}=\frac{\left(b_{n} c_{n+1}-a_{n} d_{n+1}\right) A}{a_{n+1} d_{n+1}-b_{n+1} c_{n+1}}-C_{S}, \quad z_{1}=(-1)^{n} \frac{\left(a_{n+1} b_{n}-a_{n} b_{n+1}\right) A}{a_{n+1} d_{n+1}-b_{n+1} c_{n+1}} .
$$

Since

$$
\begin{gathered}
a_{n+1} d_{n+1}-b_{n+1} c_{n+1}=(-1)^{n+1} C_{P 1} C_{P 2}\left(t_{11} t_{22}-t_{21} t_{12}\right)^{2} \tilde{\lambda}_{1}^{n} \tilde{\lambda}_{2}^{n}, \\
b_{n} c_{n+1}-a_{n} d_{n+1}=(-1)^{n} C_{P 1} C_{P 2}\left(t_{11} t_{22}-t_{12} t_{21}\right) \\
\quad \times\left(t_{11} t_{22} \tilde{\lambda}_{1}^{n} \tilde{\lambda}_{2}^{n-1}-t_{12} t_{21} \tilde{\lambda}_{1}^{n-1} \tilde{\lambda}_{2}^{n}\right),
\end{gathered}
$$


and

$$
a_{n+1} b_{n}-a_{n} b_{n+1}=C_{P 1} C_{P 2} t_{12} t_{22}\left(t_{11} t_{22}-t_{12} t_{21}\right)\left(\tilde{\lambda}_{1}^{n} \tilde{\lambda}_{2}^{n-1}-\tilde{\lambda}_{1}^{n-1} \tilde{\lambda}_{2}^{n}\right),
$$

we see that (4.37) can be simplified as

$$
y_{1}=C_{P 2} t_{12} t_{21}-C_{P 1} t_{11} t_{22}-C_{S}, \quad z_{1}=\left(C_{P 2}-C_{P 1}\right) t_{12} t_{22} .
$$

Using a similar procedure for (4.35), we have

$$
y_{2}=\left(C_{P 2}-C_{P 1}\right) t_{11} t_{21}, \quad z_{2}=C_{P 2} t_{11} t_{22}-C_{P 1} t_{12} t_{21}-C_{S} .
$$

Obviously $y_{1}, z_{1}, y_{2}$, and $z_{2}$ are independent of $n$.

After cancelling the $(n+1)$ st time derivatives in (4.34)-(4.35), we have

$$
\begin{aligned}
& \left(\frac{\partial}{\partial t}+C_{S} \frac{\partial}{\partial r}\right)\left(r \boldsymbol{u}, \boldsymbol{U}_{n m}\right)+y_{1} r\left(\hat{\boldsymbol{r}} \times \nabla \times(\hat{\boldsymbol{r}}, \boldsymbol{u}) \hat{\boldsymbol{r}}, \boldsymbol{U}_{n m}\right)+z_{1} r\left(\hat{\boldsymbol{r}} \times \nabla \times(\hat{\boldsymbol{r}}, \boldsymbol{w}) \hat{\boldsymbol{r}}, \boldsymbol{U}_{n m}\right) \\
= & -\sum_{j=1}^{n+1} \frac{j \beta_{n j}}{r^{j+1} C_{S}^{n-j}}\left(\frac{\partial}{\partial t}\right)^{n+1-j} G_{n}\left[g_{n m}^{1}\right] \\
& +\sqrt{n(n+1)} \sum_{j=1}^{n+1}\left\{\frac{(-1)^{n-j} \gamma_{n j}}{r^{j+1} A^{n-j}}\left(\frac{\partial}{\partial t}\right)^{n+1-j}\left(a_{n-j} G_{n}\left[h_{n m}^{1}\right]+b_{n-j} G_{n}\left[h_{n m}^{2}\right]\right)\right. \\
& \left.+\frac{(-1)^{n-j} C_{S} \gamma_{n j}}{r^{j+1} A^{n+1-j}}\left(\frac{\partial}{\partial t}\right)^{n+1-j}\left(a_{n+1-j} G_{n}\left[h_{n m}^{1}\right]+b_{n+1-j} G_{n}\left[h_{n m}^{2}\right]\right)\right\} \\
& +C_{S} \sqrt{n(n+1)} \sum_{j=1}^{n+1} \frac{(-1)^{n-j} j \gamma_{n, j-1}}{r^{j+1} A^{n+1-j}}\left(\frac{\partial}{\partial t}\right)^{n+1-j}\left(a_{n+1-j} G_{n}\left[h_{n m}^{1}\right]+b_{n+1-j} G_{n}\left[h_{n m}^{2}\right]\right) \\
& +y_{1} \sqrt{n(n+1)}\left\{-\sqrt{n(n+1)} \sum_{j=1}^{n+1} \frac{\gamma_{n, j-1}}{r^{j+1} C_{S}^{n+1-j}}\left(\frac{\partial}{\partial t}\right)^{n+1-j} G_{n}\left[g_{n m}^{1}\right]\right. \\
& \left.-\sum_{j=1}^{n+1} \frac{(-1)^{n+1-j}\left(\beta_{n j}+\gamma_{n, j-1}\right)}{r^{j+1} A^{n+1-j}}\left(\frac{\partial}{\partial t}\right)^{n+1-j}\left(a_{n+1-j} G_{n}\left[h_{n m}^{1}\right]+b_{n+1-j} G_{n}\left[h_{n m}^{2}\right]\right)\right\} \\
& +z_{1} \sqrt{n(n+1)}\left\{\frac{\sqrt{n(n+1)} \rho_{f}}{m} \sum_{j=1}^{n+1} \frac{\gamma_{n, j-1}}{r^{j+1} C_{S}^{n+1-j}}\left(\frac{\partial}{\partial t}\right)^{n+1-j} G_{n}\left[g_{n m}^{1}\right]\right. \\
& \left.-\sum_{j=1}^{n+1} \frac{\left(\beta_{n j}+\gamma_{n, j-1}\right)}{r^{j+1} A^{n+1-j}}\left(\frac{\partial}{\partial t}\right)^{n+1-j}\left(c_{n+1-j} G_{n}\left[h_{n m}^{1}\right]+d_{n+1-j} G_{n}\left[h_{n m}^{2}\right]\right)\right\}
\end{aligned}
$$

and

$$
\begin{aligned}
& \left(\frac{\partial}{\partial t}+C_{S} \frac{\partial}{\partial r}\right)\left(r \boldsymbol{w}, \boldsymbol{U}_{n m}\right)+y_{2} r\left(\hat{\boldsymbol{r}} \times \nabla \times(\hat{\boldsymbol{r}}, \boldsymbol{u}) \hat{\boldsymbol{r}}, \boldsymbol{U}_{n m}\right)+z_{2} r\left(\hat{\boldsymbol{r}} \times \nabla \times(\hat{\boldsymbol{r}}, \boldsymbol{w}) \hat{\boldsymbol{r}}, \boldsymbol{U}_{n m}\right) \\
= & \frac{\rho_{f}}{m} \sum_{j=1}^{n+1} \frac{j \beta_{n j}}{r^{j+1} C_{S}^{n-j}}\left(\frac{\partial}{\partial t}\right)^{n+1-j} G_{n}\left[g_{n m}^{1}\right] \\
& +\sqrt{n(n+1)} \sum_{j=1}^{n+1}\left\{\frac{\gamma_{n j}}{r^{j+1} A^{n-j}}\left(\frac{\partial}{\partial t}\right)^{n+1-j}\left(c_{n-j} G_{n}\left[h_{n m}^{1}\right]+d_{n-j} G_{n}\left[h_{n m}^{2}\right]\right)\right.
\end{aligned}
$$




$$
\begin{aligned}
& \left.-\frac{C_{S} \gamma_{n j}}{r^{j+1} A^{n+1-j}}\left(\frac{\partial}{\partial t}\right)^{n+1-j}\left(c_{n+1-j} G_{n}\left[h_{n m}^{1}\right]+d_{n+1-j} G_{n}\left[h_{n m}^{2}\right]\right)\right\} \\
& -C_{S} \sqrt{n(n+1)} \sum_{j=1}^{n+1} \frac{j \gamma_{n, j-1}}{r^{j+1} A^{n+1-j}}\left(\frac{\partial}{\partial t}\right)^{n+1-j}\left(c_{n+1-j} G_{n}\left[h_{n m}^{1}\right]+d_{n+1-j} G_{n}\left[h_{n m}^{2}\right]\right) \\
& +y_{2} \sqrt{n(n+1)}\left\{-\sqrt{n(n+1)} \sum_{j=1}^{n+1} \frac{\gamma_{n, j-1}}{r^{j+1} C_{S}^{n+1-j}}\left(\frac{\partial}{\partial t}\right)^{n+1-j} G_{n}\left[g_{n m}^{1}\right]\right. \\
& \left.-\sum_{j=1}^{n+1} \frac{(-1)^{n+1-j}\left(\beta_{n j}+\gamma_{n, j-1}\right)}{r^{j+1} A^{n+1-j}}\left(\frac{\partial}{\partial t}\right)^{n+1-j}\left(a_{n+1-j} G_{n}\left[h_{n m}^{1}\right]+b_{n+1-j} G_{n}\left[h_{n m}^{2}\right]\right)\right\} \\
& +z_{2} \sqrt{n(n+1)}\left\{\frac{\sqrt{n(n+1)} \rho_{f}}{m+1} \sum_{j=1}^{n+1} \frac{\gamma_{n, j-1}}{r^{j+1} C_{S}^{n+1-j}}\left(\frac{\partial}{\partial t}\right)^{n+1-j} G_{n}\left[g_{n m}^{1}\right]\right. \\
& \left.-\sum_{j=1}^{n+1} \frac{\left(\beta_{n j}+\gamma_{n, j-1}\right)}{r^{j+1} A^{n+1-j}}\left(\frac{\partial}{\partial t}\right)^{n+1-j}\left(c_{n+1-j} G_{n}\left[h_{n m}^{1}\right]+d_{n+1-j} G_{n}\left[h_{n m}^{2}\right]\right)\right\} .
\end{aligned}
$$

To further simplify the results above, we define vectors $\boldsymbol{\psi}_{n m}^{g^{1}}(t), \boldsymbol{\psi}_{n m}^{h^{1}}(t)$, and $\boldsymbol{\psi}_{n m}^{h^{2}}(t)$ with $n+1(j=1, \cdots, n+1)$ components by

$$
\begin{aligned}
\psi_{n m}^{g^{1}, j}(t) & =\frac{\beta_{n j}}{R \beta_{n 1} C_{S}^{n+1-j}}\left(\frac{\partial}{\partial t}\right)^{n+1-j} G_{n}\left[g_{n m}^{1}\right](R, t), \\
\psi_{n m}^{h^{1}, j}(t) & =\frac{\beta_{n j}}{R \beta_{n 1} C_{P 1}^{n+1-j}}\left(\frac{\partial}{\partial t}\right)^{n+1-j}\left\{t_{11} G_{n}\left[h_{n m}^{1}\right](R, t)+t_{12} G_{n}\left[h_{n m}^{2}\right](R, t)\right\},
\end{aligned}
$$

and

$$
\psi_{n m}^{h^{2}, j}(t)=\frac{\beta_{n j}}{R \beta_{n 1} C_{P 2}^{n+1-j}}\left(\frac{\partial}{\partial t}\right)^{n+1-j}\left\{t_{21} G_{n}\left[h_{n m}^{1}\right](R, t)+t_{22} G_{n}\left[h_{n m}^{2}\right](R, t)\right\},
$$

respectively. Since

$$
\begin{aligned}
& a_{s} G_{n}\left[h_{n m}^{1}\right]+b_{s} G_{n}\left[h_{n m}^{2}\right]=\left(t_{12} t_{21}-t_{11} t_{22}\right)^{s-1}\left\{C _ { P 1 } ^ { s } t _ { 1 2 } \left(t_{21} G_{n}\left[h_{n m}^{1}\right]\right.\right. \\
&\left.+t_{22} G_{n}\left[h_{n m}^{2}\right]-C_{P 2}^{s} t_{22}\left(t_{11} G_{n}\left[h_{n m}^{1}\right]+t_{12} G_{n}\left[h_{n m}^{2}\right]\right)\right\}
\end{aligned}
$$

and

$$
\begin{aligned}
& c_{s} G_{n}\left[h_{n m}^{1}\right]+d_{s} G_{n}\left[h_{n m}^{2}\right]=\left(t_{11} t_{22}-t_{12} t_{21}\right)^{s-1}\left\{C _ { P 1 } ^ { s } t _ { 1 1 } \left(t_{21} G_{n}\left[h_{n m}^{1}\right]\right.\right. \\
&\left.\left.+t_{22} G_{n}\left[h_{n m}^{2}\right]\right)-C_{P 2}^{s} t_{21}\left(t_{11} G_{n}\left[h_{n m}^{1}\right]+t_{12} G_{n}\left[h_{n m}^{2}\right]\right)\right\},
\end{aligned}
$$

we obtain

$$
\begin{aligned}
& \left(\frac{\partial}{\partial t}\right)^{k}\left(a_{s} G_{n}\left[h_{n m}^{1}\right]+b_{s} G_{n}\left[h_{n m}^{2}\right]\right) \\
= & \left(t_{12} t_{21}-t_{11} t_{22}\right)^{s-1} \frac{R \beta_{n 1}}{\beta_{n, n+1-k}}\left[t_{12} C_{P 1}^{s} C_{P 2}^{k} \psi_{n m}^{h^{2}, n+1-k}-t_{22} C_{P 1}^{k} C_{P 2}^{s} \psi_{n m}^{h^{1}, n+1-k}\right]
\end{aligned}
$$


and

$$
\begin{aligned}
& \left(\frac{\partial}{\partial t}\right)^{k}\left(c_{s} G_{n}\left[h_{n m}^{1}\right]+d_{s} G_{n}\left[h_{n m}^{2}\right]\right) \\
= & \left(t_{11} t_{22}-t_{12} t_{21}\right)^{s-1} \frac{R \beta_{n 1}}{\beta_{n, n+1-k}}\left[t_{11} C_{P 1}^{s} C_{P 2}^{k} \psi_{n m}^{h^{2}, n+1-k}-t_{21} C_{P 1}^{k} C_{P 2}^{s} \psi_{n m}^{h^{1}, n+1-k}\right] .
\end{aligned}
$$

Substituting (4.50)-(4.51) into (4.43)-(4.44) leads to

$$
\begin{array}{rl}
\left(\frac{\partial}{\partial t}+C_{S} \frac{\partial}{\partial r}\right)\left(r \boldsymbol{u}, \boldsymbol{U}_{n m}\right)+y_{1} & r\left(\hat{\boldsymbol{r}} \times \nabla \times(\hat{\boldsymbol{r}}, \boldsymbol{u}) \hat{\boldsymbol{r}}, \boldsymbol{U}_{n m}\right)+z_{1} r\left(\hat{\boldsymbol{r}} \times \nabla \times(\hat{\boldsymbol{r}}, \boldsymbol{w}) \hat{\boldsymbol{r}}, \boldsymbol{U}_{n m}\right) \\
& =\boldsymbol{A}_{n}^{1} \cdot \psi_{n m}^{g^{1}}(t)+\boldsymbol{A}_{n}^{2} \cdot \psi_{n m}^{h^{2}}+\boldsymbol{A}_{n}^{3} \cdot \psi_{n m}^{h^{1}}, \quad r=R, \quad
\end{array}
$$

and

$$
\begin{aligned}
\left(\frac{\partial}{\partial t}+C_{S} \frac{\partial}{\partial r}\right)\left(r \boldsymbol{w}, \boldsymbol{U}_{n m}\right)+y_{2} r & \left(\hat{\boldsymbol{r}} \times \nabla \times(\hat{\boldsymbol{r}}, \boldsymbol{u}) \hat{\boldsymbol{r}}, \boldsymbol{U}_{n m}\right)+z_{2} r\left(\hat{\boldsymbol{r}} \times \nabla \times(\hat{\boldsymbol{r}}, \boldsymbol{w}) \hat{\boldsymbol{r}}, \boldsymbol{U}_{n m}\right) \\
& =\boldsymbol{B}_{n}^{1} \cdot \psi_{n m}^{g^{1}}(t)+\boldsymbol{B}_{n}^{2} \cdot \psi_{n m}^{h^{2}}+\boldsymbol{B}_{n}^{3} \cdot \psi_{n m}^{h^{1}}, \quad r=R, \quad
\end{aligned}
$$

where $\boldsymbol{A}_{n}^{1}, \boldsymbol{A}_{n}^{2}, \boldsymbol{A}_{n}^{3}, \boldsymbol{B}_{n}^{1}, \boldsymbol{B}_{n}^{2}$, and $\boldsymbol{B}_{n}^{3}$ are the vectors with $n+1$ components defined by

$$
\begin{gathered}
A_{n}^{1, j}=\frac{n(n+1) \beta_{n 1}}{R^{j} \beta_{n j}}\left[\frac{z_{1} \rho_{f} \gamma_{n, j-1}}{M}-y_{1} \gamma_{n, j-1}-\frac{j C_{S} \beta_{n j}}{n(n+1)}\right] \\
A_{n}^{2, j}=\frac{\beta_{n 1}\left(t_{11} t_{22}-t_{12} t_{21}\right)^{n-j}}{\beta_{n j} R^{j} A^{n-j}}\left[-\sqrt{n(n+1)} \frac{t_{12} \gamma_{n j}}{t_{11} t_{22}-t_{12} t_{21}} C_{P 1}^{n-j} C_{P 2}^{n+1-j}\right. \\
\quad+\frac{t_{12} C_{S} \gamma_{n j}}{A} C_{P 1}^{n+1-j} C_{P 2}^{n+1-j}+\sqrt{n(n+1)} \frac{t_{12} j C_{S} \gamma_{n, j-1}}{A} C_{P 1}^{n+1-j} C_{P 2}^{n+1-j} \\
+y_{1} \sqrt{n(n+1)} \frac{t_{12}\left(\beta_{n j}+\gamma_{n, j-1}\right)}{A} C_{P 1}^{n+1-j} C_{P 2}^{n+1-j} \\
\left.-z_{1} \sqrt{n(n+1)} \frac{t_{11}\left(\beta_{n j}+\gamma_{n, j-1}\right)}{A} C_{P 1}^{n+1-j} C_{P 2}^{n+1-j}\right] \\
A_{n}^{3, j}=-\frac{\beta_{n 1}\left(t_{11} t_{22}-t_{12} t_{21}\right)^{n-j}}{\beta_{n j} R^{j} A^{n-j}}\left[-\sqrt{n(n+1)} \frac{t_{22} \gamma_{n j}}{t_{11} t_{22}-t_{12} t_{21}} C_{P 1}^{n+1-j} C_{P 2}^{n-j}\right. \\
\quad+\frac{t_{22} C_{S} \gamma_{n j}}{A} C_{P 1}^{n+1-j} C_{P 2}^{n+1-j}+\sqrt{n(n+1)} \frac{t_{22} j C_{S} \gamma_{n, j-1}}{A} C_{P 1}^{n+1-j} C_{P 2}^{n+1-j} \\
+y_{1} \sqrt{n(n+1)} \frac{t_{22}\left(\beta_{n j}+\gamma_{n, j-1}\right)}{A} C_{P 1}^{n+1-j} C_{P 2}^{n+1-j} \\
\left.\quad-z_{1} \sqrt{n(n+1)} \frac{t_{21}\left(\beta_{n j}+\gamma_{n, j-1}\right)}{A} C_{P 1}^{n+1-j} C_{P 2}^{n+1-j}\right] \\
B_{n}^{2, j}=\frac{\beta_{n 1}\left(t_{11} t_{22}-t_{12} t_{21}\right)^{n-j}}{\beta_{n j} R^{j} A^{n-j}}\left[\sqrt{n(n+1)} \frac{t_{11} \gamma_{n j}}{t_{11} t_{22}-t_{12} t_{21}} C_{P 1}^{n-j} C_{P 2}^{n+1-j}\right. \\
B_{n}^{1, j}=\frac{n(n+1) \beta_{n 1}}{R^{j} \beta_{n j}}\left[\frac{z_{2} \rho_{f} \gamma_{n, j-1}}{M}-y_{2} \gamma_{n, j-1}+\frac{j \rho_{f} C_{S} \beta_{n j}}{m n(n+1)}\right]
\end{gathered}
$$




$$
\begin{gathered}
-\frac{t_{11} C_{S} \gamma_{n j}}{A} C_{P 1}^{n+1-j} C_{P 2}^{n+1-j}-\sqrt{n(n+1)} \frac{t_{11} C_{S} \gamma_{n, j-1}}{A} C_{P 1}^{n+1-j} C_{P 2}^{n+1-j} \\
+y_{2} \sqrt{n(n+1)} \frac{t_{12}\left(\beta_{n j}+\gamma_{n, j-1}\right)}{A} C_{P 1}^{n+1-j} C_{P 2}^{n+1-j} \\
\left.-z_{2} \sqrt{n(n+1)} \frac{t_{11}\left(\beta_{n j}+\gamma_{n, j-1}\right)}{A} C_{P 1}^{n+1-j} C_{P 2}^{n++-j}\right] \\
B_{n}^{3, j}=-\frac{\beta_{n 1}\left(t_{11} t_{22}-t_{12} t_{21}\right)^{n-j}}{\beta_{n j} R^{j} A^{n-j}}\left[-\sqrt{n(n+1)} \frac{t_{21} \gamma_{n j}}{t_{11} t_{22}-t_{12} t_{21}} C_{P 1}^{n+1-j} C_{P 2}^{n-j}\right. \\
-\frac{t_{21} C_{S} \gamma_{n j}}{A} C_{P 1}^{n+1-j} C_{P 2}^{n+1-j}+\sqrt{n(n+1)} \frac{t_{21} C_{S} \gamma_{n, j-1}}{A} C_{P 1}^{n+1-j} C_{P 2}^{n+1-j} \\
+y_{2} \sqrt{n(n+1)} \frac{t_{22}\left(\beta_{n j}+\gamma_{n, j-1}\right)}{A} C_{P 1}^{n+1-j} C_{P 2}^{n+1-j} \\
\left.-z_{2} \sqrt{n(n+1)} \frac{t_{21}\left(\beta_{n j}+\gamma_{n, j-1}\right)}{A} C_{P 1}^{n+1-j} C_{P 2}^{n+1-j}\right] .
\end{gathered}
$$

According to the definition of $\beta, \beta_{01}=0$. In (4.45)-(4.47) and (4.54)-(4.59), for $j=1$ and $n=0$, the denominator $\beta_{n j}$ is equal to zero, but the ratio $\beta_{n j} / \beta_{n 1}$ or $\beta_{n 1} / \beta_{n j}$ is equal to 1 , so the expressions are still valid.

5. Exact boundary conditions for $\left(\hat{\boldsymbol{r}} \cdot \boldsymbol{u}, Y_{n m}\right)$ and $\left(\hat{\boldsymbol{r}} \cdot \boldsymbol{w}, Y_{n m}\right)$

To derive exact boundary conditions for $\left(\hat{\boldsymbol{r}} \cdot \boldsymbol{u}, Y_{n m}\right)$ and $\left(\hat{\boldsymbol{r}} \cdot \boldsymbol{w}, Y_{n m}\right)$, we take dot products of (2.36) and (2.37) with $\hat{\boldsymbol{r}}$, then take the inner products of the resulting expressions with $Y_{n m}$ and use lemmas 3.1 and 3.3 to get

$$
\begin{array}{r}
r\left(\hat{\boldsymbol{r}} \cdot \boldsymbol{u}, Y_{n m}\right)=-\sum_{j=0}^{n+1} \frac{\beta_{n j}+\gamma_{n, j-1}}{r^{j}}\left(-\frac{\partial}{\partial r}\right)^{n+1-j} G_{n}\left[h_{n m}^{1}\right] \\
-\sqrt{n(n+1)} \sum_{j=0}^{n} \frac{\gamma_{n j}}{r^{j+1}}\left(-\frac{\partial}{\partial r}\right)^{n-j} G_{n}\left[g_{n m}^{1}\right], \\
r\left(\hat{\boldsymbol{r}} \cdot \boldsymbol{w}, Y_{n m}\right)=-\sum_{j=0}^{n+1} \frac{\beta_{n j}+\gamma_{n, j-1}}{r^{j}}\left(-\frac{\partial}{\partial r}\right)^{n+1-j} G_{n}\left[h_{n m}^{2}\right] \\
+\frac{\sqrt{n(n+1)} \rho_{f}}{\grave{M}} \sum_{j=0}^{n} \frac{\gamma_{n j}}{r^{j+1}}\left(-\frac{\partial}{\partial r}\right)^{n-j} G_{n}\left[g_{n m}^{1}\right] .
\end{array}
$$

As the matrix $\boldsymbol{T}_{1}^{-1}$ is invertible, either $t_{11}-t_{12} \rho_{f} / \stackrel{\circ}{M} \neq 0$ or $t_{21}-t_{22} \rho_{f} / \stackrel{\circ}{M} \neq 0$ holds. If $t_{11}-t_{12} \rho_{f} / \stackrel{\circ}{M} \neq 0$, then

$$
\begin{aligned}
& t_{11} r\left(\hat{\boldsymbol{r}} \cdot \boldsymbol{u}, Y_{n m}\right)+t_{12} r\left(\hat{\boldsymbol{r}} \cdot \boldsymbol{w}, Y_{n m}\right) \\
=- & \sum_{j=0}^{n+1} \frac{\beta_{n j}+\gamma_{n, j-1}}{r^{j}}\left(-\frac{\partial}{\partial r}\right)^{n+1-j}\left(t_{11} G_{n}\left[h_{n m}^{1}\right]+t_{12} G_{n}\left[h_{n m}^{2}\right]\right) \\
& \quad-\sqrt{n(n+1)} \sum_{j=0}^{n} \frac{\gamma_{n j}}{r^{j+1}}\left(-\frac{\partial}{\partial r}\right)^{n-j}\left(t_{11} G_{n}\left[g_{n m}^{1}\right]-\frac{\rho_{f}}{\stackrel{\circ}{M}} t_{12} G_{n}\left[g_{n m}^{1}\right]\right)
\end{aligned}
$$

and

$$
t_{21} r\left(\hat{\boldsymbol{r}} \cdot \boldsymbol{u}, Y_{n m}\right)+t_{22} r\left(\hat{\boldsymbol{r}} \cdot \boldsymbol{w}, Y_{n m}\right)
$$




$$
\begin{aligned}
=-\sum_{j=0}^{n+1} \frac{\beta_{n j}+\gamma_{n, j-1}}{r^{j}}\left(-\frac{\partial}{\partial r}\right)^{n+1-j}\left(t_{21} G_{n}\left[h_{n m}^{1}\right]+t_{22} G_{n}\left[h_{n m}^{2}\right]\right) \\
\\
\quad-\sqrt{n(n+1)} \sum_{j=0}^{n} \frac{\gamma_{n j}}{r^{j+1}}\left(-\frac{\partial}{\partial r}\right)^{n-j}\left(t_{21} G_{n}\left[g_{n m}^{1}\right]-\frac{\rho_{f}}{M} t_{22} G_{n}\left[g_{n m}^{1}\right]\right) .
\end{aligned}
$$

Applying the operator $\left(\partial_{t}+C_{P 1} \partial_{r}\right)$ to (5.3), using (4.13) and (4.14), and substituting space derivatives with time derivatives, we obtain

$$
\begin{aligned}
&\left(\frac{\partial}{\partial t}+C_{P 1} \frac{\partial}{\partial r}\right)\left(t_{11} r\left(\hat{\boldsymbol{r}} \cdot \boldsymbol{u}, Y_{n m}\right)+t_{12} r\left(\hat{\boldsymbol{r}} \cdot \boldsymbol{w}, Y_{n m}\right)\right) \\
&=\sum_{j=1}^{n+1} \frac{j\left(\beta_{n j}+\gamma_{n, j-1}\right)}{r^{j+1} C_{P 1}^{n-j}}\left(\frac{\partial}{\partial t}\right)^{n+1-j}\left(t_{11} G_{n}\left[h_{n m}^{1}\right]+t_{12} G_{n}\left[h_{n m}^{2}\right]\right) \\
& \quad-\left(t_{11}-\frac{\rho_{f}}{M} t_{12}\right) \sqrt{n(n+1)} \sum_{j=0}^{n+1}\left\{\left(1-\frac{C_{P 1}}{C_{S}}\right) \frac{\gamma_{n j}}{r^{j+1} C_{S}^{n-j}}\left(\frac{\partial}{\partial t}\right)^{n+1-j} G_{n}\left[g_{n m}^{1}\right]\right. \\
&\left.-\frac{j C_{P 1} \gamma_{n, j-1}}{r^{j+1} C_{S}^{n+1-j}}\left(\frac{\partial}{\partial t}\right)^{n+1-j} G_{n}\left[g_{n m}^{1}\right]\right\} .
\end{aligned}
$$

Similarly, applying the operator $\left(\partial_{t}+C_{P 2} \partial_{r}\right)$ to $(5.4)$, we obtain

$$
\begin{aligned}
&\left(\frac{\partial}{\partial t}+\right.\left.C_{P 2} \frac{\partial}{\partial r}\right)\left(t_{21} r\left(\hat{\boldsymbol{r}} \cdot \boldsymbol{u}, Y_{n m}\right)+t_{22} r\left(\hat{\boldsymbol{r}} \cdot \boldsymbol{w}, Y_{n m}\right)\right) \\
&=\sum_{j=1}^{n+1} \frac{j\left(\beta_{n j}+\gamma_{n, j-1}\right)}{r^{j+1} C_{P 2}^{n-j}}\left(\frac{\partial}{\partial t}\right)^{n+1-j}\left(t_{21} G_{n}\left[h_{n m}^{1}\right]+t_{22} G_{n}\left[h_{n m}^{2}\right]\right) \\
& \quad-\left(t_{21}-\frac{\rho_{f}}{m} t_{22}\right) \sqrt{n(n+1)} \sum_{j=0}^{n+1}\left\{\left(1-\frac{C_{P 2}}{C_{S}}\right) \frac{\gamma_{n j}}{r^{j+1} C_{S}^{n-j}} \partial_{t}^{n+1-j} G_{n}\left[g_{n m}^{1}\right]\right. \\
&\left.-\frac{j C_{P 2} \gamma_{n, j-1}}{r^{j+1} C_{S}^{n+1-j}}\left(\frac{\partial}{\partial t}\right)^{n+1-j} G_{n}\left[g_{n m}^{1}\right]\right\} .
\end{aligned}
$$

We now seek an operator to annihilate the derivative $\partial_{t}^{n+1} G_{n}\left[g_{n m}^{1}\right]$.

In polar coordinates, we denote $\boldsymbol{u}=\left(u^{r}, u^{\vartheta}, u^{\phi}\right)$ and $\boldsymbol{u}^{\tan }=\left(0, u^{\vartheta}, u^{\phi}\right)$. Since $\nabla$. $\boldsymbol{V}_{n m}=0$, we have

$$
\begin{aligned}
& r \nabla \cdot \boldsymbol{u}_{n m}^{t a n}=\left(\frac{\sqrt{n(n+1)}}{r} \frac{\partial}{\partial r}\left(r g_{n m}^{1}\right)-\frac{n(n+1)}{r} h_{n m}^{1}\right) Y_{n m}, \\
& r \nabla \cdot \boldsymbol{w}_{n m}^{t a n}=\left(\frac{\sqrt{n(n+1)}}{r} \frac{\partial}{\partial r}\left(r g_{n m}^{2}\right)-\frac{n(n+1)}{r} h_{n m}^{2}\right) Y_{n m} .
\end{aligned}
$$

Taking the inner product of (5.7) and (5.8) with $Y_{n m}$, respectively, and by using lemmas 3.1 and 3.3 , we obtain

$$
\begin{array}{r}
\left(r \nabla \cdot \boldsymbol{u}^{t a n}, Y_{n m}\right)=-\sqrt{n(n+1)} \sum_{j=0}^{n+1} \frac{\beta_{n j}}{r^{j+1} C_{S}^{n+1-j}}\left(\frac{\partial}{\partial t}\right)^{n+1-j} G_{n}\left[g_{n m}^{1}\right] \\
-n(n+1) \sum_{j=1}^{n+1} \frac{\gamma_{n, j-1}}{r^{j+1}}\left(-\frac{\partial}{\partial r}\right)^{n+1-j} G_{n}\left[h_{n m}^{1}\right]
\end{array}
$$


and

$$
\begin{array}{r}
\left(r \nabla \cdot \boldsymbol{w}^{t a n}, Y_{n m}\right)=\frac{\sqrt{n(n+1)} \rho_{f}}{M} \sum_{j=0}^{n+1} \frac{\beta_{n j}}{r^{j+1} C_{S}^{n+1-j}}\left(\frac{\partial}{\partial t}\right)^{n+1-j} G_{n}\left[g_{n m}^{1}\right] \\
-n(n+1) \sum_{j=1}^{n+1} \frac{\gamma_{n, j-1}}{r^{j+1}}\left(-\frac{\partial}{\partial r}\right)^{n+1-j} G_{n}\left[h_{n m}^{2}\right] .
\end{array}
$$

The terms $\left(-\partial_{r}\right)^{n+1-j} G_{n}\left[h_{n m}^{1}\right]$ and $\left(-\partial_{r}\right)^{n+1-j} G_{n}\left[h_{n m}^{2}\right]$ in (5.9)-(5.10) cannot be replaced by time derivatives directly. We need to proceed further. Notice that we have

$$
\begin{aligned}
& t_{11}\left(r \nabla \cdot \boldsymbol{u}^{t a n}, Y_{n m}\right)+t_{12}\left(r \nabla \cdot \boldsymbol{w}^{t a n}, Y_{n m}\right) \\
& =\sqrt{n(n+1)}\left(\frac{\rho_{f}}{\stackrel{\circ}{M}} t_{12}-t_{11}\right) \sum_{j=0}^{n+1} \frac{\beta_{n j}}{r^{j+1} C_{S}^{n+1-j}}\left(\frac{\partial}{\partial t}\right)^{n+1-j} G_{n}\left[g_{n m}^{1}\right] \\
& \quad-n(n+1) \sum_{j=1}^{n+1} \frac{\gamma_{n, j-1}}{r^{j+1} C_{P 1}^{n+1-j}}\left(\frac{\partial}{\partial t}\right)^{n+1-j}\left(t_{11} G_{n}\left[h_{n m}^{1}\right]+t_{12} G_{n}\left[h_{n m}^{2}\right]\right), \\
& t_{21}\left(r \nabla \cdot \boldsymbol{u}^{t a n}, Y_{n m}\right)+t_{22}\left(r \nabla \cdot \boldsymbol{w}^{t a n}, Y_{n m}\right) \\
& =\sqrt{n(n+1)}\left(\frac{\rho_{f}}{\stackrel{\circ}{M}} t_{22}-t_{21}\right) \sum_{j=0}^{n+1} \frac{\beta_{n j}}{r^{j+1} C_{S}^{n+1-j}}\left(\frac{\partial}{\partial t}\right)^{n+1-j} G_{n}\left[g_{n m}^{1}\right] \\
& \quad-n(n+1) \sum_{j=1}^{n+1} \frac{\gamma_{n, j-1}}{r^{j+1} C_{P 2}^{n+1-j}}\left(\frac{\partial}{\partial t}\right)^{n+1-j}\left(t_{21} G_{n}\left[h_{n m}^{1}\right]+t_{22} G_{n}\left[h_{n m}^{2}\right]\right) .
\end{aligned}
$$

We recall by definition that $\gamma_{n,-1}=0$ and $\gamma_{n 0}=\beta_{n 0}=1$. Comparing the term $\partial_{t}^{n+1} G_{n}\left[g_{n m}^{1}\right]$ in (5.5) and (5.11), we see that they are the same up to a factor of $C_{S}-C_{P 1}$. Multiplying (5.11) with $C_{P 1}-C_{S}$ and adding the resulting expression to (5.5) to cancel $\partial_{t}^{n+1} G_{n}\left[g_{n m}^{1}\right]$, we obtain

$$
\begin{aligned}
&\left(\frac{\partial}{\partial t}+\right.\left.C_{P 1} \frac{\partial}{\partial r}\right)\left[t_{11} r\left(\hat{\boldsymbol{r}} \cdot \boldsymbol{u}, Y_{n m}\right)+t_{12} r\left(\hat{\boldsymbol{r}} \cdot \boldsymbol{w}, Y_{n m}\right)\right] \\
&-\left(C_{S}-C_{P 1}\right)\left[t_{11}\left(r \nabla \cdot \boldsymbol{u}^{t a n}, Y_{n m}\right)+t_{12}\left(r \nabla \cdot \boldsymbol{w}^{t a n}, Y_{n m}\right)\right] \\
&=\sum_{j=1}^{n+1} \frac{j\left(\beta_{n j}+\gamma_{n, j-1}\right)}{r^{j+1} C_{P 1}^{n-j}}\left(\frac{\partial}{\partial t}\right)^{n+1-j}\left(t_{11} G_{n}\left[h_{n m}^{1}\right]+t_{12} G_{n}\left[h_{n m}^{2}\right]\right) \\
&-\left(t_{11}-\frac{\rho_{f}}{M} t_{12}\right) \sqrt{n(n+1)} \sum_{j=1}^{n+1}\left\{\left(1-\frac{C_{P 1}}{C_{S}}\right) \frac{\gamma_{n j}}{r^{j+1} C_{S}^{n-j}}\left(\frac{\partial}{\partial t}\right)^{n+1-j} G_{n}\left[g_{n m}^{1}\right]\right. \\
&\left.-\frac{j C_{P 1} \gamma_{n, j-1}}{r^{j+1} C_{S}^{n+1-j}}\left(\frac{\partial}{\partial t}\right)^{n+1-j} G_{n}\left[g_{n m}^{1}\right]\right\} \\
&-\left(C_{S}-C_{P 1}\right)\left\{\sqrt{n(n+1)}\left(\frac{\rho_{f}}{m} t_{12}-t_{11}\right) \sum_{j=1}^{n+1} \frac{\beta_{n j}}{r^{j+1} C_{S}^{n+1-j}}\left(\frac{\partial}{\partial t}\right)^{n+1-j} G_{n}\left[g_{n m}^{1}\right]\right. \\
&\left.-n(n+1) \sum_{j=1}^{n+1} \frac{\gamma_{n, j-1}}{r^{j+1} C_{P 1}^{n+1-j}}\left(\frac{\partial}{\partial t}\right)^{n+1-j}\left(t_{11} G_{n}\left[h_{n m}^{1}\right]+t_{12} G_{n}\left[h_{n m}^{2}\right]\right)\right\} . \quad
\end{aligned}
$$


Similarly, multiplying (5.12) with $C_{P 2}-C_{S}$ and adding the resulting expression to (5.6) to cancel $\partial_{t}^{n+1} G_{n}\left[g_{n m}^{1}\right]$, we obtain

$$
\begin{aligned}
&\left(\frac{\partial}{\partial t}+\right.\left.C_{P 2} \frac{\partial}{\partial r}\right)\left[t_{21} r\left(\hat{\boldsymbol{r}} \cdot \boldsymbol{u}, Y_{n m}\right)+t_{22} r\left(\hat{\boldsymbol{r}} \cdot \boldsymbol{w}, Y_{n m}\right)\right] \\
&-\left(C_{S}-C_{P 2}\right)\left[t_{21}\left(r \nabla \cdot \boldsymbol{u}^{t a n}, Y_{n m}\right)+t_{22}\left(r \nabla \cdot \boldsymbol{w}^{t a n}, Y_{n m}\right)\right] \\
&=\sum_{j=1}^{n+1} \frac{j\left(\beta_{n j}+\gamma_{n, j-1}\right)}{r^{j+1} C_{P 2}^{n-j}}\left(\frac{\partial}{\partial t}\right)^{n+1-j}\left(t_{21} G_{n}\left[h_{n m}^{1}\right]+t_{22} G_{n}\left[h_{n m}^{2}\right]\right) \\
&-\left(t_{21}-\frac{\rho_{f}}{M} t_{22}\right) \sqrt{n(n+1)} \sum_{j=1}^{n+1}\left\{\left(1-\frac{C_{P 2}}{C_{S}}\right) \frac{\gamma_{n j}}{r^{j+1} C_{S}^{n-j}}\left(\frac{\partial}{\partial t}\right)^{n+1-j} G_{n}\left[g_{n m}^{1}\right]\right. \\
&\left.-\frac{j C_{P 2} \gamma_{n, j-1}}{r^{j+1} C_{S}^{n+1-j}}\left(\frac{\partial}{\partial t}\right)^{n+1-j} G_{n}\left[g_{n m}^{1}\right]\right\} \\
&-\left(C_{S}-C_{P 2}\right)\left\{\sqrt{n(n+1)}\left(\frac{\rho_{f}}{m} t_{22}-t_{21}\right) \sum_{j=1}^{n+1} \frac{\beta_{n j}}{r^{j+1} C_{S}^{n+1-j}}\left(\frac{\partial}{\partial t}\right)^{n+1-j} G_{n}\left[g_{n m}^{1}\right]\right. \\
&\left.-n(n+1) \sum_{j=1}^{n+1} \frac{\gamma_{n, j-1}}{r^{j+1} C_{P 2}^{n+1-j}}\left(\frac{\partial}{\partial t}\right)^{n+1-j}\left(t_{21} G_{n}\left[h_{n m}^{1}\right]+t_{22} G_{n}\left[h_{n m}^{2}\right]\right)\right\} . \quad(5.14)
\end{aligned}
$$

We simplify the expressions (5.13) and (5.14) further:

$$
\begin{gathered}
\left(\frac{\partial}{\partial t}+C_{P 1} \frac{\partial}{\partial r}\right)\left[t_{11} r\left(\hat{\boldsymbol{r}} \cdot \boldsymbol{u}, Y_{n m}\right)\right. \\
\left.+t_{12} r\left(\hat{\boldsymbol{r}} \cdot \boldsymbol{w}, Y_{n m}\right)\right]-\left(C_{S}-C_{P 1}\right)\left[t_{11}\left(r \nabla \cdot \boldsymbol{u}^{t a n}, Y_{n m}\right)+t_{12}\left(r \nabla \cdot \boldsymbol{w}^{t a n}, Y_{n m}\right)\right] \\
=\boldsymbol{C}_{n}^{1} \cdot \boldsymbol{\psi}_{n m}^{h^{1}}(t)+\boldsymbol{C}_{n}^{2} \cdot \boldsymbol{\psi}_{n m}^{g^{1}}(t), r=R \\
\left(\frac{\partial}{\partial t}+C_{P 2} \frac{\partial}{\partial r}\right)\left[t_{21} r\left(\hat{\boldsymbol{r}} \cdot \boldsymbol{u}, Y_{n m}\right)+t_{22} r\left(\hat{\boldsymbol{r}} \cdot \boldsymbol{w}, Y_{n m}\right)\right] \\
\quad-\left(C_{S}-C_{P 2}\right)\left[t_{21}\left(r \nabla \cdot \boldsymbol{u}^{t a n}, Y_{n m}\right)+t_{22}\left(r \nabla \cdot \boldsymbol{w}^{t a n}, Y_{n m}\right)\right] \\
=\boldsymbol{D}_{n}^{1} \cdot \boldsymbol{\psi}_{n m}^{h^{2}}(t)+\boldsymbol{D}_{n}^{2} \cdot \boldsymbol{\psi}_{n m}^{g^{1}}(t), r=R,
\end{gathered}
$$

where $\boldsymbol{C}_{n}^{1}, \boldsymbol{C}_{n}^{2}, \boldsymbol{D}_{n}^{1}, \boldsymbol{D}_{n}^{2}$ are the vectors with the $n+1$ components defined by

$$
\begin{aligned}
& C_{n}^{1, j}= \frac{\beta_{n 1}}{\beta_{n j}}\left[\frac{j C_{P 1}\left(\beta_{n j}+\gamma_{n, j-1}\right)}{R^{j}}+n(n+1) \frac{\left(C_{S}-C_{P 1}\right) \gamma_{n, j-1}}{R^{j}}\right], \\
& C_{n}^{2, j}=\left(\frac{\rho_{f}}{M} t_{12}-t_{11}\right) \frac{\sqrt{n(n+1)} \beta_{n 1}}{R^{j} \beta_{n j}}\left[\left(C_{S}-C_{P 1}\right) \gamma_{n j}\right. \\
&\left.\quad-j C_{P 1} \gamma_{n, j-1}-\left(C_{S}-C_{P 1}\right) \beta_{n j}\right], \\
& D_{n}^{1, j}=\frac{\beta_{n 1}}{\beta_{n j}}\left[\frac{j C_{P 2}\left(\beta_{n j}+\gamma_{n, j-1}\right)}{R^{j}}+n(n+1) \frac{\left(C_{S}-C_{P 2}\right) \gamma_{n, j-1}}{R^{j}}\right], \\
& D_{n}^{2, j}=\left(\frac{\rho_{f}}{M} t_{22}-t_{21}\right) \frac{\sqrt{n(n+1)} \beta_{n 1}}{R^{j} \beta_{n j}}\left[\left(C_{S}-C_{P 2}\right) \gamma_{n j}\right. \\
&\left.\quad-j C_{P 2} \gamma_{n, j-1}-\left(C_{S}-C_{P 2}\right) \beta_{n j}\right] .
\end{aligned}
$$


6. Ordinary differential equations for $\boldsymbol{\psi}_{n m}^{g^{1}}(t), \boldsymbol{\psi}_{n m}^{h^{1}}(t)$, and $\boldsymbol{\psi}_{n m}^{h^{2}}(t)$

The boundary equations (4.52)-(4.53) and (5.15)-(5.16) involve $\boldsymbol{\psi}_{n m}^{g^{1}}(t), \boldsymbol{\psi}_{n m}^{h^{1}}(t)$, and $\boldsymbol{\psi}_{n m}^{h^{2}}(t)$. By the definitions of $\boldsymbol{\psi}_{n m}^{g^{1}}(t), \boldsymbol{\psi}_{n m}^{h^{1}}(t)$, and $\boldsymbol{\psi}_{n m}^{h^{2}}(t)$, they involve highorder time derivatives. So we need multi-step methods to solve them, which results in large computational costs. This difficulty can be solved by using the following three ordinary differential equations for $\boldsymbol{\psi}_{n m}^{g^{1}}(t), \boldsymbol{\psi}_{n m}^{h^{1}}(t)$, and $\boldsymbol{\psi}_{n m}^{h^{2}}(t)$.

By the definitions of $\boldsymbol{\psi}_{n m}^{g^{1}}(t), \boldsymbol{\psi}_{n m}^{h^{1}}(t)$, and $\boldsymbol{\psi}_{n m}^{h^{2}}(t)$ in (4.45)-(4.47), the following expressions hold:

$$
\begin{aligned}
& \frac{1}{C_{S}} \frac{d}{d t} \psi_{n m}^{g^{1}, j}(R, t)=\frac{\beta_{n j}}{\beta_{n, j-1}} \psi_{n m}^{g^{1}, j-1}, \quad j=2,3, \cdots, n+1, \\
& \frac{1}{C_{P 1}} \frac{d}{d t} \psi_{n m}^{h^{1}, j}(R, t)=\frac{\beta_{n j}}{\beta_{n, j-1}} \psi_{n m}^{h^{1}, j-1}, \quad j=2,3, \cdots, n+1, \\
& \frac{1}{C_{P 2}} \frac{d}{d t} \psi_{n m}^{h^{2}, j}(R, t)=\frac{\beta_{n j}}{\beta_{n, j-1}} \psi_{n m}^{h^{2}, j-1}, \quad j=2,3, \cdots, n+1 .
\end{aligned}
$$

First, we consider the following:

$$
\begin{aligned}
& t_{11} R\left(\boldsymbol{u}, \boldsymbol{U}_{n m}\right)+t_{12} R\left(\boldsymbol{w}, \boldsymbol{U}_{n m}\right) \\
= & \left(t_{11}-\frac{\rho_{f}}{M} t_{12}\right) \sum_{j=0}^{n+1} \frac{\beta_{n 1}}{R^{j-1}} \psi_{n m}^{g^{1}, j}+\sqrt{n(n+1)} \sum_{j=1}^{n+1} \frac{\beta_{n 1} \gamma_{n, j-1}}{\beta_{n j} R^{j-1}} \psi_{n m}^{h^{1}, j} .
\end{aligned}
$$

Moving the term of $\left(\frac{1}{C_{S}} \partial_{t}\right)^{n+1} G_{n}\left[g_{n m}^{1}\right]$ on the right hand side in (6.4) to the left yields

$$
\begin{aligned}
& \left(\frac{1}{C_{S}} \frac{\partial}{\partial t}\right)^{n+1} G_{n}\left[g_{n m}^{1}\right] \\
=- & \sum_{j=1}^{n+1} \frac{\beta_{n 1}}{R^{j-1}} \psi_{n m}^{g^{1}, j}-\frac{\sqrt{n(n+1)}}{t_{11}-\rho_{f} t_{12} / \stackrel{\circ}{M}} \sum_{j=1}^{n+1} \frac{\beta_{n 1} \gamma_{n, j-1}}{\beta_{n j} R^{j-1}} \psi_{n m}^{h^{1}, j} \\
& +\frac{t_{11}}{t_{11}-\rho_{f} t_{12} / \stackrel{\circ}{M}} R\left(\boldsymbol{u}, \boldsymbol{U}_{n m}\right)+\frac{t_{12}}{t_{11}-\rho_{f} t_{12} / \stackrel{M}{M}} R\left(\boldsymbol{w}, \boldsymbol{U}_{n m}\right) .
\end{aligned}
$$

Thus

$$
\begin{aligned}
\frac{1}{C_{S}} \frac{d}{d t} \psi_{n m}^{g^{1}, 1}(R, t)=- & \sum_{j=1}^{n+1} \frac{\beta_{n 1}}{R^{j}} \psi_{n m}^{g^{1}, j}-\frac{\sqrt{n(n+1)}}{t_{11}-\rho_{f} t_{12} / \stackrel{\circ}{M}} \sum_{j=1}^{n+1} \frac{\beta_{n 1} \gamma_{n, j-1}}{\beta_{n j} R^{j}} \psi_{n m}^{h^{1}, j} \\
& +\frac{t_{11}}{t_{11}-\rho_{f} t_{12} / \stackrel{\circ}{M}}\left(\boldsymbol{u}, \boldsymbol{U}_{n m}\right)+\frac{t_{12}}{t_{11}-\rho_{f} t_{12} / \stackrel{\circ}{M}}\left(\boldsymbol{w}, \boldsymbol{U}_{n m}\right) .
\end{aligned}
$$

Using (6.1) and (6.6), we obtain the first-order ordinary differential equation for $\boldsymbol{\psi}_{n m}^{g^{1}}(t)$ :

$$
\begin{aligned}
\frac{1}{C_{S}} \frac{d}{d t} \boldsymbol{\psi}_{n m}^{g^{1}}(t)=\boldsymbol{S}_{n} \boldsymbol{\psi}_{n m}^{g^{1}}(t)+\left[\frac{t_{11}}{t_{11}-\rho_{f} t_{12} / \stackrel{\circ}{M}}\left(\boldsymbol{u}, \boldsymbol{U}_{n m}\right)\right. \\
\left.+\frac{t_{12}}{t_{11}-\rho_{f} t_{12} / \stackrel{\circ}{M}}\left(\boldsymbol{w}, \boldsymbol{U}_{n m}\right)-\boldsymbol{z}_{n} \cdot \boldsymbol{\psi}_{n m}^{h^{1}}(t)\right] \boldsymbol{e}_{n+1},
\end{aligned}
$$

where $\boldsymbol{S}_{n}=\left\{S_{n}^{i j}\right\}$ is the $(n+1) \times(n+1)$ constant matrix

$$
S_{n}^{i j}= \begin{cases}-n(n+1) /\left(2 R^{j}\right), & i=1, \\ \beta_{n i} /\left(\beta_{n j}\right), & i=j+1, \\ 0, & \text { otherwise, }\end{cases}
$$


and $\boldsymbol{z}_{n}=\left\{z_{n}^{j}\right\}$ is the constant vector with $n+1$ components defined by

$$
z_{n}^{j}=\frac{\sqrt{n(n+1)} \gamma_{n, j-1} \beta_{n 1}}{\left(t_{11}-\rho_{f} t_{12} / M\right) R^{j} \beta_{n j}}, \quad j=1, \cdots, n+1 .
$$

Now considering the following linear combination with $r\left(\hat{\boldsymbol{r}} \cdot \boldsymbol{u}, Y_{n m}\right)$ and $r(\hat{\boldsymbol{r}}$. $\left.\boldsymbol{w}, Y_{n m}\right)$ and setting $r=R$, we obtain

$$
\begin{aligned}
& t_{11} r\left(\hat{\boldsymbol{r}} \cdot \boldsymbol{u}, Y_{n m}\right)+t_{12} r\left(\hat{\boldsymbol{r}} \cdot \boldsymbol{w}, Y_{n m}\right) \\
=- & \sum_{j=1}^{n+1} \frac{\beta_{n 1}\left(\beta_{n j}+\gamma_{n, j-1}\right)}{\beta_{n j} R^{j-1}} \psi_{n m}^{h^{1}, j}+\left(\frac{\rho_{f} t_{12}}{m}-t_{11}\right) \sqrt{n(n+1)} \sum_{j=1}^{n+1} \frac{\beta_{n 1} \gamma_{n, j-1}}{\beta_{n j} R^{j-1}} \psi_{n m}^{g^{1}, j} \\
& \quad-\left(\frac{1}{C_{P 1}} \frac{\partial}{\partial t}\right)^{n+1}\left(t_{11} G_{n}\left[h_{n m}^{1}\right]+t_{12} G_{n}\left[h_{n m}^{2}\right]\right), \quad r=R .
\end{aligned}
$$

Moving the term $-\left(\frac{1}{C_{P 1}} \partial_{t}\right)^{n+1}\left(t_{11} G_{n}\left[h_{n m}^{1}\right]+t_{12} G_{n}\left[h_{n m}^{2}\right]\right)$ on the right hand side in (6.10) to the left yields

$$
\begin{aligned}
& \left(\frac{1}{C_{P 1}} \frac{\partial}{\partial t}\right)^{n+1}\left(t_{11} G_{n}\left[h_{n m}^{1}\right]+t_{12} G_{n}\left[h_{n m}^{2}\right]\right) \\
= & -\sum_{j=1}^{n+1} \frac{\beta_{n 1}\left(\beta_{n j}+\gamma_{n, j-1}\right)}{\beta_{n j} R^{j-1}} \psi_{n m}^{h^{1}, j}+\left(\frac{\rho_{f} t_{12}}{m}-t_{11}\right) \sqrt{n(n+1)} \sum_{j=1}^{n+1} \frac{\beta_{n 1} \gamma_{n, j-1}}{\beta_{n j} R^{j-1}} \psi_{n m}^{g^{1}, j} \\
& \quad-t_{11} r\left(\hat{\boldsymbol{r}} \cdot \boldsymbol{u}, Y_{n m}\right)-t_{12} r\left(\hat{\boldsymbol{r}} \cdot \boldsymbol{w}, Y_{n m}\right), \quad r=R .
\end{aligned}
$$

Therefore

$$
\begin{aligned}
& \frac{1}{C_{P 1}} \frac{d}{d t} \psi_{n m}^{h^{1}, 1}(r, t) \\
= & \frac{1}{R C_{P 1}^{n+1}}\left(\frac{\partial}{\partial t}\right)^{n+1}\left(t_{11} G_{n}\left[h_{n m}^{1}\right]+t_{12} G_{n}\left[h_{n m}^{2}\right]\right) \\
= & -\sum_{j=1}^{n+1} \frac{\beta_{n 1}\left(\beta_{n j}+\gamma_{n, j-1}\right)}{\beta_{n j} R^{j}} \psi_{n m}^{h^{1}, j}+\left(\frac{\rho_{f} t_{12}}{m}-t_{11}\right) \sqrt{n(n+1)} \sum_{j=1}^{n+1} \frac{\beta_{n 1} \gamma_{n, j-1}}{\beta_{n j} R^{j}} \psi_{n m}^{g^{1}, j} \\
& \quad-t_{11}\left(\hat{\boldsymbol{r}} \cdot \boldsymbol{u}, Y_{n m}\right)-t_{12}\left(\hat{\boldsymbol{r}} \cdot \boldsymbol{w}, Y_{n m}\right), \quad r=R .
\end{aligned}
$$

By (6.2) and (6.12), we obtain the first-order ordinary differential equation for $\boldsymbol{\psi}_{n m}^{h^{1}}(t)$ :

$$
\begin{array}{r}
\frac{1}{C_{P 1}} \frac{d}{d t} \boldsymbol{\psi}_{n m}^{h^{1}}(t)=\tilde{\boldsymbol{S}}_{n} \boldsymbol{\psi}_{n m}^{h^{1}}(t)+\left\{\left(\frac{\rho_{f} t_{12}}{m}-t_{11}\right) \sqrt{n(n+1)} \tilde{\boldsymbol{z}}_{n} \cdot \boldsymbol{\psi}_{n m}^{g^{1}}(t)\right. \\
\left.-t_{11}\left(\hat{\boldsymbol{r}} \cdot \boldsymbol{u}, Y_{n m}\right)-t_{12}\left(\hat{\boldsymbol{r}} \cdot \boldsymbol{w}, Y_{n m}\right)\right\} \boldsymbol{e}_{n+1}, \quad r=R,
\end{array}
$$

where $\tilde{\boldsymbol{S}}_{n}=\left\{\tilde{S}_{n}^{i j}\right\}$ is the $(n+1) \times(n+1)$ constant matrix

$$
\tilde{S}_{n}^{i j}= \begin{cases}-\beta_{n 1}\left(\beta_{n j}+\gamma_{n, j-1}\right) /\left(\beta_{n j} R^{j}\right), & i=1, \\ \beta_{n i} /\left(\beta_{n j}\right), & i=j+1, \\ 0, & \text { otherwise }\end{cases}
$$

$\tilde{\boldsymbol{z}}_{n}=\left\{\tilde{z}_{n}^{j}\right\}$ is the constant vector with $n+1$ components defined by

$$
\tilde{z}_{n}^{j}=\frac{\beta_{n 1} \gamma_{n, j-1}}{\beta_{n j} R^{j}}, \quad j=1, \cdots, n+1
$$


and $\boldsymbol{e}_{n+1}=[1,0, \cdots, 0]^{T}$ is the $n+1$ dimensional constant vector. Using the same procedure, we obtain the first-order ordinary differential equation for the vector $\boldsymbol{\psi}_{n m}^{h^{2}}(t)$ :

$$
\begin{aligned}
& \frac{1}{C_{P 2}} \frac{d}{d t} \boldsymbol{\psi}_{n m}^{h^{2}}(t)=\tilde{\boldsymbol{S}}_{n} \boldsymbol{\psi}_{n m}^{h^{2}}(t)+\left\{\left(\frac{\rho_{f} t_{22}}{m}-t_{21}\right) \sqrt{n(n+1)} \tilde{\boldsymbol{z}}_{n} \cdot \boldsymbol{\psi}_{n m}^{g^{1}}(t)\right. \\
&\left.-t_{21}\left(\hat{\boldsymbol{r}} \cdot \boldsymbol{u}, Y_{n m}\right)-t_{22}\left(\hat{\boldsymbol{r}} \cdot \boldsymbol{w}, Y_{n m}\right)\right\} \boldsymbol{e}_{n+1}, \quad r=R .
\end{aligned}
$$

7. Exact nonreflecting boundary conditions for $u$ and $\boldsymbol{w}$

In this section, we will derive the exact nonreflecting boundary conditions for the displacements $\boldsymbol{u}$ and $\boldsymbol{w}$ based on the results in the previous sections.

For the radial components, we multiply (5.15) and (5.16) with $Y_{n m}$, respectively, and sum over $n, m$ to obtain

$$
\begin{aligned}
& \left(\frac{\partial}{\partial t}+C_{P 1} \frac{\partial}{\partial r}\right)\left(t_{11} r \hat{\boldsymbol{r}} \cdot \boldsymbol{u}+t_{12} r \hat{\boldsymbol{r}} \cdot \boldsymbol{w}\right)-\left(C_{S}-C_{P 1}\right)\left(t_{11} r \nabla \cdot \boldsymbol{u}^{t a n}+t_{12} r \nabla \cdot \boldsymbol{w}^{t a n}\right) \\
= & \sum_{n \geq 0} \sum_{|m| \leq n}\left[\boldsymbol{C}_{n}^{1} \cdot \boldsymbol{\psi}_{n m}^{h^{1}}(t)+\boldsymbol{C}_{n}^{2} \cdot \boldsymbol{\psi}_{n m}^{g^{1}}(t)\right] Y_{n m}, \quad r=R, \\
& \left(\frac{\partial}{\partial t}+C_{P 2} \frac{\partial}{\partial r}\right)\left(t_{21} r \hat{\boldsymbol{r}} \cdot \boldsymbol{u}+t_{22} r \hat{\boldsymbol{r}} \cdot \boldsymbol{w}\right)-\left(C_{S}-C_{P 2}\right)\left(t_{21} r \nabla \cdot \boldsymbol{u}^{t a n}+t_{22} r \nabla \cdot \boldsymbol{w}^{t a n}\right) \\
= & \sum_{n \geq 0} \sum_{|m| \leq n}\left[\boldsymbol{D}_{n}^{1} \cdot \boldsymbol{\psi}_{n m}^{h^{2}}(t)+\boldsymbol{D}_{n}^{2} \cdot \boldsymbol{\psi}_{n m}^{g^{1}}(t)\right] Y_{n m}, \quad r=R .
\end{aligned}
$$

For the tangential components, we first multiply (3.15) by $\boldsymbol{V}_{n m}$ and (4.52) by $\boldsymbol{U}_{n m}$, and sum over $n, m$. Then we add the two resulting expressions. Noticing $(r \boldsymbol{u}, \hat{\boldsymbol{r}}) \neq 0$ and $\left(r \boldsymbol{u}^{\tan }, \hat{\boldsymbol{r}}\right)=0$, and

$$
\left(r \boldsymbol{u}, \boldsymbol{U}_{n m}\right)=\left(r \boldsymbol{u}^{\tan }, \boldsymbol{U}_{n m}\right), \quad\left(r \boldsymbol{u}, \boldsymbol{V}_{n m}\right)=\left(r \boldsymbol{u}^{\tan }, \boldsymbol{V}_{n m}\right),
$$

and the fact that $y_{1}$ and $z_{1}$ are constants, independent of $n$ by Theorem 4.2, we obtain the result for $\boldsymbol{u}^{\text {tan }}$ :

$$
\begin{gathered}
\left(\frac{\partial}{\partial t}+C_{S} \frac{\partial}{\partial r}\right) r \boldsymbol{u}^{t a n}+y_{1} r \hat{\boldsymbol{r}} \times \nabla \times(\hat{\boldsymbol{r}} \cdot \boldsymbol{u}) \hat{\boldsymbol{r}}+z_{1} r \hat{\boldsymbol{r}} \times \nabla \times(\hat{\boldsymbol{r}} \cdot \boldsymbol{w}) \hat{\boldsymbol{r}} \\
=\sum_{n \geq 1} \sum_{|m| \leq n}\left[\boldsymbol{A}_{n}^{1} \cdot \boldsymbol{\psi}_{n m}^{g^{1}}(t)+\boldsymbol{A}_{n}^{2} \cdot \boldsymbol{\psi}_{n m}^{h^{2}}(t)+\boldsymbol{A}_{n}^{3} \cdot \boldsymbol{\psi}_{n m}^{h^{1}}(t)\right] \boldsymbol{U}_{n m} \\
-C_{S} \sum_{n \geq 1} \sum_{|m| \leq n}\left[\boldsymbol{l}_{n} \cdot \boldsymbol{\psi}_{n m}^{f^{1}}(t)\right] \boldsymbol{V}_{n m}, \quad r=R .
\end{gathered}
$$

With a similar procedure, we obtain the result for $\boldsymbol{w}^{\tan }$ :

$$
\begin{gathered}
\left(\frac{\partial}{\partial t}+C_{S} \partial_{r}\right) r \boldsymbol{w}^{t a n}+y_{2} r \hat{\boldsymbol{r}} \times \nabla \times(\hat{\boldsymbol{r}} \cdot \boldsymbol{u}) \hat{\boldsymbol{r}}+z_{2} r \hat{\boldsymbol{r}} \times \nabla \times(\hat{\boldsymbol{r}} \cdot \boldsymbol{w}) \hat{\boldsymbol{r}} \\
=\sum_{n \geq 1} \sum_{|m| \leq n}\left[\boldsymbol{B}_{n}^{1} \cdot \boldsymbol{\psi}_{n m}^{g^{1}}(t)+\boldsymbol{B}_{n}^{2} \cdot \boldsymbol{\psi}_{n m}^{h^{2}}(t)+\boldsymbol{B}_{n}^{3} \cdot \boldsymbol{\psi}_{n m}^{h^{1}}(t)\right] \boldsymbol{U}_{n m} \\
+\frac{\rho_{f} C_{S}}{\stackrel{\varrho}{M}} \sum_{n \geq 1} \sum_{|m| \leq n}\left[\boldsymbol{l}_{n} \cdot \boldsymbol{\psi}_{n m}^{f^{1}}(t)\right] \boldsymbol{V}_{n m}, \quad r=R .
\end{gathered}
$$

The vector functions $\boldsymbol{\psi}_{n m}^{f^{1}}, \boldsymbol{\psi}_{n m}^{g^{1}}, \boldsymbol{\psi}_{n m}^{h^{1}}$, and $\boldsymbol{\psi}_{n m}^{h^{2}}$ satisfy the following first-order, linear, ordinary differential equations:

$$
\frac{1}{C_{S}} \frac{d}{d t} \boldsymbol{\psi}_{n m}^{f^{1}}(t)=\boldsymbol{A}_{n} \boldsymbol{\psi}_{n m}^{f^{1}}+\left(\left.\boldsymbol{u}\right|_{r=R}, \boldsymbol{V}_{n m}\right) \boldsymbol{e}_{n}, \quad \boldsymbol{\psi}_{n m}^{f^{1}}(0)=0
$$




$$
\begin{aligned}
& \frac{1}{C_{S}} \frac{d}{d t} \boldsymbol{\psi}_{n m}^{g^{1}}(t)=\boldsymbol{S}_{n} \boldsymbol{\psi}_{n m}^{g^{1}}(t)+\left[-\boldsymbol{z}_{n} \cdot \boldsymbol{\psi}_{n m}^{h^{1}}(t)+\frac{t_{11}}{t_{11}-\rho_{f} t_{12} / m}\left(\left.\boldsymbol{u}\right|_{r=R}, \boldsymbol{U}_{n m}\right)\right. \\
& \left.+\frac{t_{12}}{t_{11}-\rho_{f} t_{12} / \stackrel{\circ}{M}}\left(\left.\boldsymbol{w}\right|_{r=R}, \boldsymbol{U}_{n m}\right)\right] \boldsymbol{e}_{n+1}, \quad \boldsymbol{\psi}_{n m}^{g^{1}}(0)=0 \\
& \frac{1}{C_{P 1}} \frac{d}{d t} \boldsymbol{\psi}_{n m}^{h^{1}}(t)=\tilde{\boldsymbol{S}}_{n} \boldsymbol{\psi}_{n m}^{h^{1}}(t)+\left[\left(\frac{\rho_{f} t_{12}}{\stackrel{\circ}{M}}-t_{11}\right) \sqrt{n(n+1)} \tilde{\boldsymbol{z}}_{n} \cdot \boldsymbol{\psi}_{n m}^{g^{1}}(t)\right. \\
& \left.-t_{11}\left(\hat{\boldsymbol{r}} \cdot \boldsymbol{u}, Y_{n m}\right)-t_{12}\left(\hat{\boldsymbol{r}} \cdot \boldsymbol{w}, Y_{n m}\right)\right] \boldsymbol{e}_{n+1}, \quad \boldsymbol{\psi}_{n m}^{h^{1}}(0)=0 \\
& \frac{1}{C_{P 2}} \frac{d}{d t} \boldsymbol{\psi}_{n m}^{h^{2}}(t)=\tilde{\boldsymbol{S}}_{n} \boldsymbol{\psi}_{n m}^{h^{2}}(t)+\left[\left(\frac{\rho_{f} t_{22}}{\stackrel{\circ}{M}}-t_{21}\right) \sqrt{n(n+1)} \tilde{\boldsymbol{z}}_{n} \cdot \boldsymbol{\psi}_{n m}^{g^{1}}(t)\right. \\
& \left.-t_{21}\left(\hat{\boldsymbol{r}} \cdot \boldsymbol{u}, Y_{n m}\right)-t_{22}\left(\hat{\boldsymbol{r}} \cdot \boldsymbol{w}, Y_{n m}\right)\right] \boldsymbol{e}_{n+1}, \quad \boldsymbol{\psi}_{n m}^{h^{2}}(0)=0
\end{aligned}
$$

Finally we combine (7.1) and (7.3), (7.2) and (7.4), respectively, into a single nonreflecting boundary conditions for $\boldsymbol{u}$ and $\boldsymbol{w}$ at $\mathfrak{B}$ :

$$
\begin{gathered}
{\left[\left(\frac{\partial}{\partial t}+C_{P 1} \frac{\partial}{\partial r}\right)\left(t_{11} r \hat{\boldsymbol{r}} \cdot \boldsymbol{u}+t_{12} r \hat{\boldsymbol{r}} \cdot \boldsymbol{w}\right)-\left(C_{S}-C_{P 1}\right)\left(t_{11} r \nabla \cdot \boldsymbol{u}^{t a n}+t_{12} r \nabla \cdot \boldsymbol{w}^{t a n}\right)\right] \hat{\boldsymbol{r}}} \\
+\left(\frac{\partial}{\partial t}+C_{S} \frac{\partial}{\partial r}\right) r \boldsymbol{u}^{t a n}+y_{1} r \hat{\boldsymbol{r}} \times \nabla \times(\hat{\boldsymbol{r}}, \boldsymbol{u}) \hat{\boldsymbol{r}}+z_{1} r \hat{\boldsymbol{r}} \times \nabla \times(\hat{\boldsymbol{r}}, \boldsymbol{w}) \hat{\boldsymbol{r}} \\
=\sum_{n \geq 1} \sum_{|m| \leq n}\left[\boldsymbol{A}_{n}^{1} \cdot \boldsymbol{\psi}_{n m}^{g^{1}}(t)+\boldsymbol{A}_{n}^{2} \cdot \boldsymbol{\psi}_{n m}^{h^{2}}+\boldsymbol{A}_{n}^{3} \cdot \boldsymbol{\psi}_{n m}^{h^{1}}\right] \boldsymbol{U}_{n m}-C_{S} \sum_{n \geq 1} \sum_{|m| \leq n}\left[\boldsymbol{l}_{n} \cdot \boldsymbol{\psi}_{n m}^{f^{1}}(t)\right] \boldsymbol{V}_{n m} \\
\quad+\sum_{n \geq 0} \sum_{|m| \leq n}\left[\boldsymbol{C}_{n}^{1} \cdot \boldsymbol{\psi}_{n m}^{h^{1}}(t)+\boldsymbol{C}_{n}^{2} \cdot \boldsymbol{\psi}_{n m}^{g^{1}}(t)\right] Y_{n m} \hat{\boldsymbol{r}}, \quad r=R
\end{gathered}
$$

and

$$
\begin{gathered}
{\left[\left(\frac{\partial}{\partial t}+C_{P 2} \frac{\partial}{\partial r}\right)\left(t_{21} r \hat{\boldsymbol{r}} \cdot \boldsymbol{u}+t_{22} r \hat{\boldsymbol{r}} \cdot \boldsymbol{w}\right)-\left(C_{S}-C_{P 2}\right)\left(t_{21} r \nabla \cdot \boldsymbol{u}^{t a n}+t_{22} r \nabla \cdot \boldsymbol{w}^{\tan }\right)\right] \hat{\boldsymbol{r}}} \\
\quad+\left(\frac{\partial}{\partial t}+C_{S} \frac{\partial}{\partial r}\right) r \boldsymbol{w}^{t a n}+y_{2} r \hat{\boldsymbol{r}} \times \nabla \times(\hat{\boldsymbol{r}}, \boldsymbol{u}) \hat{\boldsymbol{r}}+z_{2} r \hat{\boldsymbol{r}} \times \nabla \times(\hat{\boldsymbol{r}}, \boldsymbol{w}) \hat{\boldsymbol{r}} \\
=\sum_{n \geq 1} \sum_{|m| \leq n}\left[\boldsymbol{B}_{n}^{1} \cdot \boldsymbol{\psi}_{n m}^{g^{1}}(t)+\boldsymbol{B}_{n}^{2} \cdot \boldsymbol{\psi}_{n m}^{h^{2}}+\boldsymbol{B}_{n}^{3} \cdot \boldsymbol{\psi}_{n m}^{h^{1}}\right] \boldsymbol{U}_{n m} \\
\quad+\frac{\rho_{f} C_{S}}{M} \sum_{n \geq 1} \sum_{|m| \leq n}\left[\boldsymbol{l}_{n} \cdot \boldsymbol{\psi}_{n m}^{f^{1}}(t)\right] \boldsymbol{V}_{n m} \\
\quad+\sum_{n \geq 0} \sum_{|m| \leq n}\left[\boldsymbol{D}_{n}^{1} \cdot \boldsymbol{\psi}_{n m}^{h^{2}}(t)+\boldsymbol{D}_{n}^{2} \cdot \boldsymbol{\psi}_{n m}^{g^{1}}(t)\right] Y_{n m} \hat{\boldsymbol{r}}, \quad r=R .
\end{gathered}
$$

In (7.9)-(7.10), the vector $\boldsymbol{l}_{\boldsymbol{n}}$ is given by (3.14). The vectors $\boldsymbol{A}_{n}^{1}, \boldsymbol{A}_{n}^{2}, \boldsymbol{A}_{n}^{3}, \boldsymbol{B}_{n}^{1}, \boldsymbol{B}_{n}^{2}$, and $\boldsymbol{B}_{n}^{3}$ are defined in (4.55)-(4.59), and the vectors $\boldsymbol{C}_{n}^{1}, \boldsymbol{C}_{n}^{2}, \boldsymbol{D}_{n}^{1}, \boldsymbol{D}_{n}^{2}$ are defined in (5.17)-(5.20). $y_{1}, z_{1}, y_{2}$, and $z_{2}$ are given in (4.41)-(4.42). According to the discussions in Section 4 , it is not difficult to know that $t_{i j}$ can be determined by the following expressions:

$$
t_{11}=\frac{1}{1-s_{1} s_{2}}, \quad t_{12}=-\frac{s_{2}}{1-s_{1} s_{2}}
$$




$$
t_{21}=-\frac{s_{1}}{1-s_{1} s_{2}}, \quad t_{22}=\frac{1}{1-s_{1} s_{2}},
$$

where

$$
s_{1}=\frac{\lambda_{1}-\left(\stackrel{\circ}{M} \lambda+2 \stackrel{\circ}{M} \mu-\alpha \rho_{f} M\right)}{\alpha M \stackrel{\circ}{M}-\rho_{f} M}, \quad s_{2}=\frac{\lambda_{2}-\left(\rho M-\alpha \rho_{f} M\right)}{\alpha \rho M-\rho_{f} \lambda-2 \rho_{f} \mu},
$$

and

$$
\begin{gathered}
\lambda_{1}=\frac{Q_{1}+\sqrt{Q_{2}}}{2}, \quad \lambda_{2}=\frac{Q_{1}-\sqrt{Q_{2}}}{2}, \\
Q_{1}=\stackrel{\circ}{M} \lambda+2 \stackrel{\circ}{M} \mu+\rho M-2 \alpha \rho_{f} M, \\
Q_{2}=\left(\stackrel{\circ}{M} \lambda+2 \stackrel{\circ}{M} \mu+\rho M-2 \alpha \rho_{f} M\right)^{2}-4\left[\left(\stackrel{\circ}{M} \lambda+2 \stackrel{\circ}{M} \mu-\alpha \rho_{f} M\right)\right. \\
\left.\times\left(\rho M-\alpha \rho_{f} M\right)-\left(\alpha \rho M-\rho_{f} \lambda-2 \rho_{f} M\right)\left(\alpha \stackrel{\circ}{M} M-\rho_{f} M\right)\right] .
\end{gathered}
$$

\section{Numerical computations}

In this section we present some numerical results to illustrate the effects of our boundary conditions derived above. A grid is defined on the computational domain $\mathcal{B}$ with mesh sizes $\Delta r=R / N_{r}, \Delta \vartheta=\pi / N_{\vartheta}$, and $\Delta \phi=2 \pi / N_{\phi}$ in the directions $\hat{\boldsymbol{r}}, \hat{\boldsymbol{\vartheta}}$, and $\hat{\boldsymbol{\phi}}$ respectively. The value of the component $u^{r}(r, \vartheta, \phi, t)$ at a general grid point and at a time $n \Delta t$ is denoted by $u_{(i, j, k, n)}^{r}$, where $\Delta t$ is the time step and $i=0,1,2, \cdots, N_{r} ; j=0,1, \cdots, N_{\vartheta} ; k=0,1, \cdots, N_{\phi}-1$. Similar notations are also used for the other components $u^{\vartheta}, u^{\phi}, w^{r}, w^{\vartheta}$, and $w^{\phi}$. We will use the classical second-order finite difference scheme to discretize the equations. In the following, we present the finite difference schemes for $u^{r}, u^{\vartheta}, u^{\phi}, w^{r}, w^{\vartheta}$, and $w^{\phi}$ $\left(j=1, \cdots, N_{\vartheta}-1 ; \quad k=0,1, \cdots, N_{\phi}-1\right)$ :

$$
\begin{aligned}
& u_{\left(N_{r}, j, k, n+1\right)}^{r} \\
& =u_{\left(N_{r}, j, k, n\right)}^{r}+\frac{\Delta t}{i \Delta r\left(t_{11} t_{22}-t_{12} t_{21}\right)}\left\{t_{22} \sum_{0 \leq n \leq N} \sum_{|m| \leq n}\left[\boldsymbol{C}_{n}^{1} \cdot \boldsymbol{\psi}_{n m}^{h^{1}}+\boldsymbol{C}_{n}^{2} \cdot \boldsymbol{\psi}_{n m}^{g^{1}}\right] Y_{n m}\right. \\
& \quad-t_{12} \sum_{0 \leq n \leq N|m| \leq n}\left[\boldsymbol{D}_{n}^{1} \cdot \boldsymbol{\psi}_{n m}^{h^{2}}+\boldsymbol{D}_{n}^{2} \cdot \boldsymbol{\psi}_{n m}^{g^{1}}\right] Y_{n m}+\left(C_{P 2}-C_{P 1}\right) t_{12} t_{22} \\
& \times\left[w_{\left(N_{r}, j, k, n\right)}^{r}+i \gamma_{r} w_{\left(N_{r}-1, j, k, n\right)}^{r}\right]+\left(C_{P 2} t_{12} t_{21}-C_{P 1} t_{11} t_{22}\right) \\
& \times\left[u_{\left(N_{r}, j, k, n\right)}^{r}+i \gamma_{r} u_{\left(N_{r}-1, j, k, n\right)}^{r}\right]+\left[\left(C_{S}-C_{P 1}\right) t_{11} t_{22}-\left(C_{S}-C_{P 2}\right) t_{12} t_{21}\right] \\
& \times\left[\frac{u_{\left(N_{r}, j+1, k, n\right)}^{\vartheta}-u_{\left(N_{r}, j-1, k, n\right)}^{\vartheta}+\frac{\cos \vartheta}{\sin \vartheta} u_{\left(N_{r}, j, k, n\right)}^{\vartheta}}{2 \Delta \vartheta}\right. \\
& \left.\quad+\frac{1}{\sin \vartheta} \frac{u_{\left(N_{r}, j, k+1, n\right)}^{\phi}-u_{\left(N_{r}, j, k-1, n\right)}^{\phi}}{2 \Delta \phi}\right] \\
& +\left(C_{P 2}-C_{P 1}\right) t_{12} t_{22}\left[\frac{w_{\left(N_{r}, j+1, k, n\right)}^{\vartheta}-w_{\left(N_{r}, j-1, k, n\right)}^{\vartheta}}{2 \Delta \vartheta}+\frac{\cos \vartheta}{\sin \vartheta} w_{\left(N_{r}, j, k, n\right)}^{\vartheta}\right. \\
& \left.\left.+\frac{1}{\sin \vartheta} \frac{w_{\left(N_{r}, j, k+1, n\right)}^{\phi}-w_{\left(N_{r}, j, k-1, n\right)}^{\phi}}{2 \Delta \phi}\right]\right\},
\end{aligned}
$$




$$
\begin{aligned}
u_{\left(N_{r}, j, k, n+1\right)}^{\vartheta} & =\frac{\Delta t}{i \Delta r}\left\{\sum_{0 \leq n \leq N} \sum_{\left(N_{r}, j, k, n\right)}\left[\boldsymbol{A}_{n}^{1} \cdot \boldsymbol{\psi}_{n m}^{g^{1}}+\boldsymbol{A}_{n}^{2} \cdot \boldsymbol{\psi}_{n m}^{h^{2}}+\boldsymbol{A}_{n}^{3} \cdot \boldsymbol{\psi}_{n m}^{h^{1}}\right]\right. \\
& \times \frac{1}{\sqrt{n(n+1)}} \frac{\partial Y_{n m}}{\partial \vartheta}+\frac{C_{S}}{\sin \vartheta} \sum_{0 \leq n \leq N} \sum_{|m| \leq n}\left(\boldsymbol{l}_{n} \cdot \boldsymbol{\psi}_{n m}^{f^{1}}\right) \frac{1}{\sqrt{n(n+1)}} \frac{\partial Y_{n m}}{\partial \phi} \\
& -y \frac{u_{\left(N_{r}, j+1, k, n\right)}^{r}-u_{\left(N_{r}, j-1, k, n\right)}^{r}}{2 \Delta \vartheta}-z_{1} \frac{w_{\left(N_{r}, j+1, k, n\right)}^{r}-w_{\left(N_{r}, j-1, k, n\right)}^{r}}{2 \Delta \vartheta} \\
& -C_{S}\left[u_{\left(N_{r}, j, k, n\right)}^{\vartheta}+i\left(u_{\left(N_{r}, j, k, n\right)}^{\vartheta}-u_{\left(N_{r}-1, j, k, n\right)}^{\vartheta}\right]\right\}
\end{aligned}
$$

$$
\begin{aligned}
u_{\left(N_{r}, j, k, n+1\right)}^{\phi} & +\frac{\Delta t}{i \Delta r}\left\{\frac{1}{\sin \vartheta} \sum_{0 \leq n \leq N} \sum_{\left(N_{r}, j, k, n\right)}\left(\boldsymbol{A}_{n}^{1} \cdot \boldsymbol{\psi}_{n m}^{g^{1}}+\boldsymbol{A}_{n}^{2} \cdot \boldsymbol{\psi}_{n m}^{h^{2}}+\boldsymbol{A}_{n}^{3} \cdot \boldsymbol{\psi}_{n m}^{h^{1}}\right)\right. \\
& \times \frac{1}{\sqrt{n(n+1)}} \frac{\partial Y_{n m}}{\partial \phi}-C_{S} \sum_{0 \leq n \leq N|m| \leq n} \sum_{n}\left(\boldsymbol{l}_{n} \cdot \boldsymbol{\psi}_{n m}^{f^{1}}\right) \frac{1}{\sqrt{n(n+1)}} \frac{\partial Y_{n m}}{\partial \vartheta} \\
& -\frac{y_{1}}{\sin \vartheta} \frac{u_{\left(N_{r}, j, k+1, n\right)}^{r}-u_{\left(N_{r}, j, k-1, n\right)}^{r}}{2 \Delta \phi}-\frac{z_{1}}{\sin \vartheta} \frac{w_{\left(N_{r}, j, k+1, n\right)}^{r}-w_{\left(N_{r}, j, k-1, n\right)}^{r}}{2 \Delta \phi} \\
& -C_{S}\left[u_{\left(N_{r}, j, k, n\right)}^{\phi}+i\left(u_{\left(N_{r}, j, k, n\right)}^{\phi}-u_{\left(N_{r}-1, j, k, n\right)}^{\phi}\right]\right\},
\end{aligned}
$$

$$
\begin{aligned}
w_{\left(N_{r}, j, k, n+1\right)}^{r} & =w_{\left(N_{r}, j, k, n\right)}^{r}+\frac{\Delta t}{i \Delta r\left(t_{11} t_{22}-t_{12} t_{21}\right)}\left\{t_{11} \sum_{0 \leq n \leq N} \sum_{|m| \leq n}\left(\boldsymbol{D}_{n}^{1} \cdot \boldsymbol{\psi}_{n m}^{h^{2}}+\boldsymbol{D}_{n}^{2} \cdot \boldsymbol{\psi}_{n m}^{g^{1}}\right) Y_{n m}\right. \\
& -t_{21} \sum_{0 \leq n \leq N|m| \leq n} \sum_{n}\left(\boldsymbol{C}_{n}^{1} \cdot \boldsymbol{\psi}_{n m}^{h^{1}}+\boldsymbol{C}_{n}^{2} \cdot \boldsymbol{\psi}_{n m}^{g^{1}}\right) Y_{n m} \\
& +\left(C_{P 1}-C_{P 2}\right) t_{11} t_{21}\left[u_{\left(N_{r}, j, k, n\right)}^{r}+i\left(u_{\left(N_{r}, j, k, n\right)}^{r}-u_{\left(N_{r}-1, j, k, n\right)}^{r}\right)\right] \\
& +\left(C_{P 1} t_{12} t_{21}-C_{P 2} t_{11} t_{22}\right)\left[w_{\left(N_{r}, j, k, n\right)}^{r}+i\left(w_{\left(N_{r}, j, k, n\right)}^{r}-w_{\left(N_{r}-1, j, k, n\right)}^{r}\right)\right] \\
& +\left[\left(C_{S}-C_{P 2}\right) t_{11} t_{22}-\left(C_{S}-C_{P 1}\right) t_{12} t_{21}\right]\left[\frac{w_{\left(N_{r}, j+1, k, n\right)}^{\vartheta}-w_{\left(N_{r}, j-1, k, n\right)}^{\vartheta}}{2 \Delta \vartheta}\right. \\
& \left.+\frac{\cos \vartheta}{\sin \vartheta} w_{\left(N_{r}, j, k, n\right)}^{\vartheta}+\frac{1}{\sin \vartheta} \frac{w_{\left(N_{r}, j, k+1, n\right)}^{\phi}-w_{\left(N_{r}, j, k-1, n\right)}^{\phi}}{2 \Delta \phi}\right] \\
& +\left(C_{P 1}-C_{P 2}\right) t_{11} t_{21}\left[\frac{u_{\left(N_{r}, j+1, k, n\right)}^{\vartheta}-u_{\left(N_{r}, j-1, k, n\right)}^{\vartheta}}{2 \Delta \vartheta}\right. \\
& \left.\left.+\frac{\cos \vartheta}{\sin \vartheta} u_{\left(N_{r}, j, k, n\right)}^{\vartheta}+\frac{1}{\sin \vartheta} \frac{u_{\left(N_{r}, j, k+1, n\right)}^{\phi}-u_{\left(N_{r}, j, k-1, n\right)}^{\phi}}{2 \Delta \phi}\right]\right\}
\end{aligned}
$$

$w_{\left(N_{r}, j, k, n+1\right)}^{\vartheta}$ 


$$
\begin{aligned}
=w_{\left(N_{r}, j, k, n\right)}^{\vartheta} & +\frac{\Delta t}{i \Delta r}\left\{\sum_{0 \leq n \leq N} \sum_{|m| \leq n}\left(\boldsymbol{B}_{n}^{1} \cdot \boldsymbol{\psi}_{n m}^{g^{1}}+\boldsymbol{B}_{n}^{2} \cdot \boldsymbol{\psi}_{n m}^{h^{2}}+\boldsymbol{B}_{n}^{3} \cdot \boldsymbol{\psi}_{n m}^{h^{1}}\right)\right. \\
& \times \frac{1}{\sqrt{n(n+1)}} \frac{\partial Y_{n m}}{\partial \vartheta}-\frac{C_{S} \rho_{f}}{\sin \vartheta} \sum_{0 \leq n \leq N} \sum_{|m| \leq n}\left(\boldsymbol{l}_{n} \cdot \boldsymbol{\psi}_{n m}^{f^{1}}\right) \frac{1}{\sqrt{n(n+1)}} \frac{\partial Y_{n m}}{\partial \phi} \\
& -y_{2} \frac{u_{\left(N_{r}, j+1, k, n\right)}^{r}-u_{\left(N_{r}, j-1, k, n\right)}^{r}}{2 \Delta \vartheta}-z_{2} \frac{w_{\left(N_{r}, j+1, k, n\right)}^{r}-w_{\left(N_{r}, j-1, k, n\right)}^{r}}{2 \Delta \vartheta} \\
& \left.-C_{S}\left[w_{\left(N_{r}, j, k, n\right)}^{\vartheta}+i\left(w_{\left(N_{r}, j, k, n\right)}^{\vartheta}-w_{\left(N_{r}-1, j, k, n\right)}^{\vartheta}\right)\right]\right\}
\end{aligned}
$$

$$
\begin{aligned}
w_{\left(N_{r}, j, k, n+1\right)}^{\phi} & =\frac{\Delta t}{i \Delta r}\left\{\frac{1}{\sin \vartheta} \sum_{\left(N_{r}, j, k, n\right)}+\sum_{0 \leq n \leq N \mid \leq n}\left(\boldsymbol{B}_{n}^{1} \cdot \boldsymbol{\psi}_{n m}^{g^{1}}+\boldsymbol{B}_{n}^{2} \cdot \boldsymbol{\psi}_{n m}^{h^{2}}+\boldsymbol{B}_{n}^{3} \cdot \boldsymbol{\psi}_{n m}^{h^{1}}\right)\right. \\
& \times \frac{1}{\sqrt{n(n+1)}} \frac{\partial Y_{n m}}{\partial \phi}-\frac{C_{S} \rho_{f}}{m} \sum_{0 \leq n \leq N} \sum_{|m| \leq n}\left(\boldsymbol{l}_{n} \cdot \boldsymbol{\psi}_{n m}^{f^{1}}\right) \frac{1}{\sqrt{n(n+1)}} \frac{\partial Y_{n m}}{\partial \vartheta} \\
& -\frac{y_{2}}{\sin \vartheta} \frac{u_{\left(N_{r}, j, k+1, n\right)}^{r}-u_{\left(N_{r}, j, k-1, n\right)}^{r}}{2 \Delta \phi}-\frac{z_{2}}{\sin \vartheta} \frac{w_{\left(N_{r}, j, k+1, n\right)}^{r}-w_{\left(N_{r}, j, k-1, n\right)}^{r}}{2 \Delta \phi} \\
& \left.-C_{S}\left[w_{\left(N_{r}, j, k, n\right)}^{\phi}+i\left(w_{\left(N_{r}, j, k, n\right)}^{\phi}-w_{\left(N_{r}-1, j, k, n\right)}^{\phi}\right)\right]\right\},
\end{aligned}
$$

We remark that, for our numerical computations, the sums over $n$ on the right side of (7.9) and (7.10) need to be truncated so that $n$ varies between 0 and some finite value $N$. For the examples shown below, we have used $N=3$ in (8.1)-(8.6). We will see that this choice of $N$ is good enough to produce significant absorbing effects. The computational complexity on the boundary $\mathfrak{B}$ per time step, since we are using the finite difference method, is $O\left(N^{2} N_{\vartheta} N_{\phi}\right)$. For large values of $N$ the amount of computational work can be reduced by using the fast discrete polynomial transform $[20,51]$. In our computations, we choose $N_{r}=40, N_{\vartheta}=20, N_{\phi}=40$, and $\Delta t=0.0001$. The source function is defined as

$$
f=\delta\left(r-r_{0}, \vartheta-\vartheta_{0}, \phi-\phi_{0}\right)\left(\sin (100 t) e^{-2500 t^{2}}, 0,0\right),
$$

where $\left(r_{0}, \vartheta_{0}, \phi_{0}\right)$ is the position of the source. For clarity, the source is placed at the center. Such a source will produce wavefronts of concentric circles which are easy to identify. The CFL stability condition used in our computations is

$$
\frac{\Delta t}{\Delta r} \leq \frac{1}{\sqrt{C_{P 1}^{2}+C_{P 2}^{2}+C_{s}^{2}}} .
$$

The above three velocities can be obtained from the physical parameters of the porous medium. In our simulations, the three velocities $C_{P 1}, C_{P 2}$, and $C_{S}$ are $3106 \mathrm{~m} / \mathrm{s}$, $893 \mathrm{~m} / \mathrm{s}$, and $1616 \mathrm{~m} / \mathrm{s}$, respectively. The other physical parameters are listed in table 8.1. The dimension of $K_{f}, K_{s}, K_{b}, \mu$, and $\lambda$ is $10^{10} \mathrm{~Pa}$. Figure 8.1-8.3 are the snapshots of the component $u_{x}$ at times $0.25 s, 0.28 s$, and $0.30 s$ in Cartesian coordinates, respectively. For the components $u_{y}$ and $u_{z}$ the phenomena are similar, we omit them to save space. Our analysis shows that the fast compressional wave arrives the boundary at $0.193 \mathrm{~s}$. In the figure $8.1(\mathrm{a})$, figure $8.2(\mathrm{a})$, and figure $8.3(\mathrm{a})$, we present the solutions computed without using our exact nonreflecting boundary condition, 
and in this case, the Dirichlet boundary condition is used. In the figure 8.1(b), figure $8.2(\mathrm{~b})$, and figure $8.3(\mathrm{~b})$, we present the solutions computed by using our exact nonreflecting boundary condition. Notice that all figures in this paper have the same color scale. We see, from the figure (a), that there are artificial reflections from the boundary, while from the figure (b), we see almost no artificial reflections. From these results, we see that the exact nonreflecting boundary condition is a very effective way to absorb artificial boundary reflections. For this model the runtime of 50 time steps is about $70 \mathrm{~s}$ on our PC with a $2.1 \mathrm{MHz} \mathrm{CPU}$. Moreover, we have tested our method for different values of $N$. In particular, for the use of $N=1,3,5$, the method produces almost identical solutions and the numeric values of the $L_{2}$ norm errors for these cases are also almost identical. Since these differences are difficult to observe in the figures, we will only report the results for the case $N=3$. On the other hand, the ratio of the computational times for the cases $N=1,3,5$ is about 1:4:15. In the case of using large values of $N$, the technique of the fast transform described in $[20,51]$ is useful. We remark that it is an interesting but a difficult problem to give a general theoretical quantitative analysis of the relationship between the $L_{2}$ norm errors and the optimal $N$.

\begin{tabular}{ccccccccc}
\hline$K_{f}$ & $\rho_{f}\left(\mathrm{~kg} / \mathrm{m}^{3}\right)$ & $K s(\mathrm{~Pa})$ & $\rho_{s}\left(\mathrm{~kg} / \mathrm{m}^{3}\right)$ & $K_{b}$ & $\mu$ & $\lambda$ & $a$ & $\phi$ \\
\hline 2.4 & 1040 & 3.5 & 2650 & 4.17 & 1.855 & 1.215 & 2167 & 0.3 \\
\hline
\end{tabular}

TABLE 8.1. Physical properties of the model used in numerical computations.

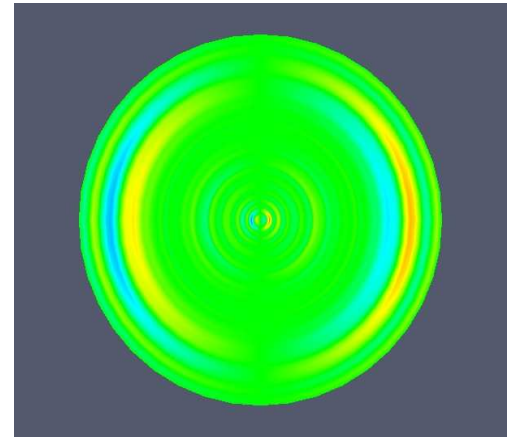

(a)

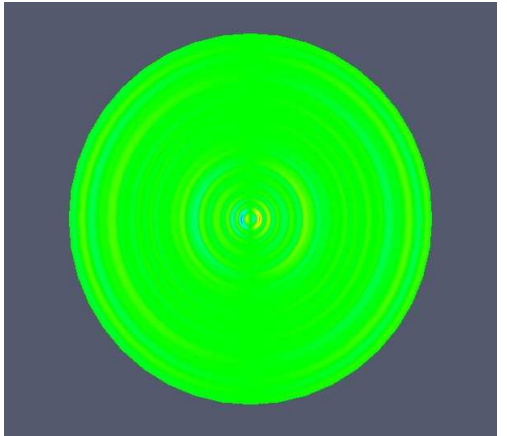

(b)

FIG. 8.1. Snapshot of wave propagation of $u_{x}$ at $t=0.25 \mathrm{~s}$ without (a) and with (b) exact absorbing boundary conditions.

When the position of the source $(r, \vartheta, \phi)$ is placed at $(0,0,0)$, the wavefronts are all concentric circles. This source is usually called the ball cavity source. We now consider numerical simulations with a source function that is not located at the center. The source is now distributed within a small volume where it's center is located at a point half radius away from the sphere center. This source is called the volume source. We use the volume source instead of the ball cavity source as the later is tedious to describe in mathematical terms in this case. Figure 8.4 shows the snapshots of the behavior of $u_{x}$ at $t=0.10 \mathrm{~s}$ computed without (a) and with (b) the exact nonreflecting boundary condition. We can see that both figure 8.4(a) and figure 8.4(b) are the same 


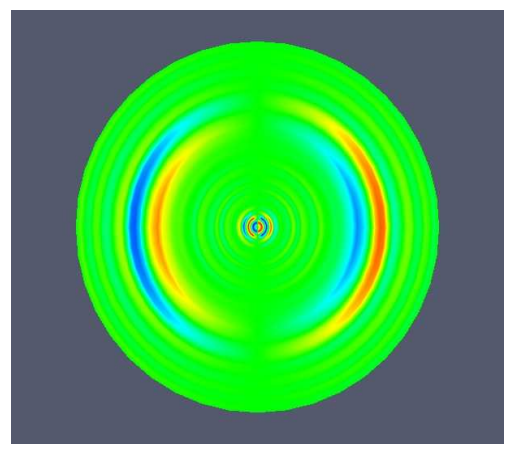

(a)

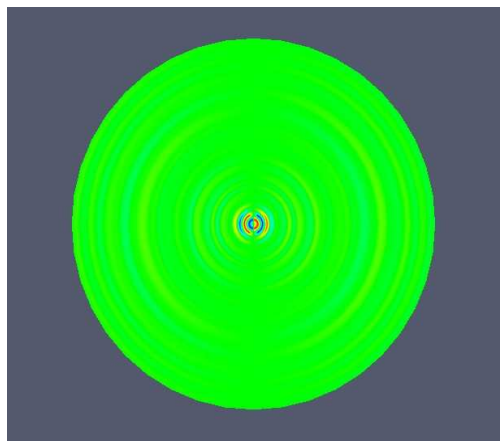

(b)

FIG. 8.2. Snapshot of wave propagation of $u_{x}$ at $t=0.28 \mathrm{~s}$ without (a) and with (b) exact absorbing boundary conditions.

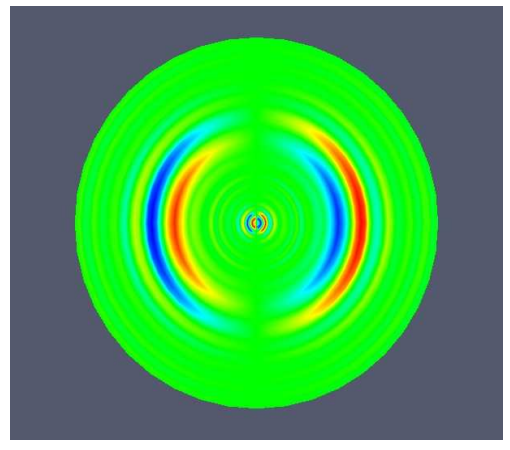

(a)

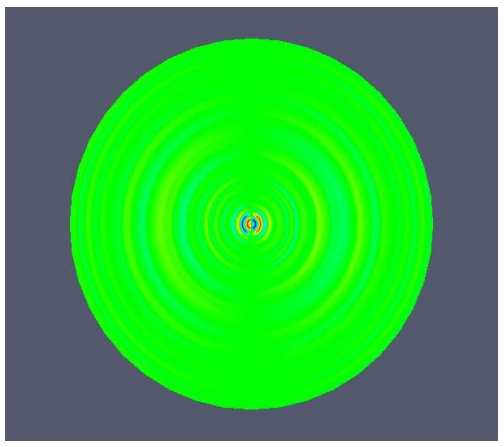

(b)

FIG. 8.3. Snapshot of wave propagation of $u_{x}$ at $t=0.30 s$ without (a) and with (b) exact absorbing boundary conditions.

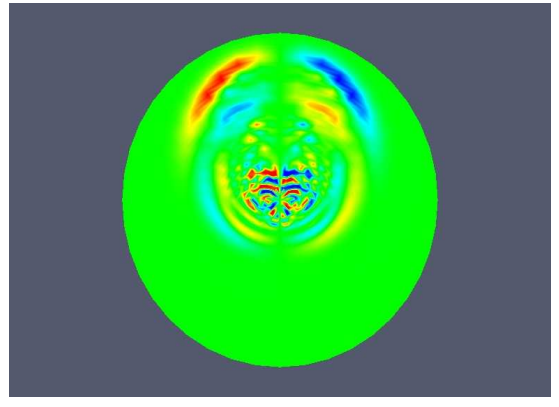

$(a)$

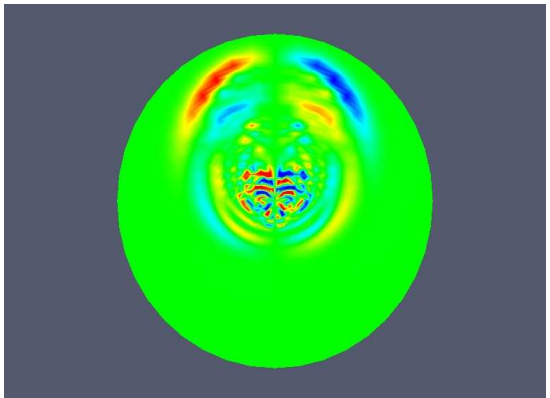

(b)

FIG. 8.4. Snapshot of wave propagation of $u_{x}$ at $t=0.1$ s without (a) and with (b) exact absorbing boundary conditions. 


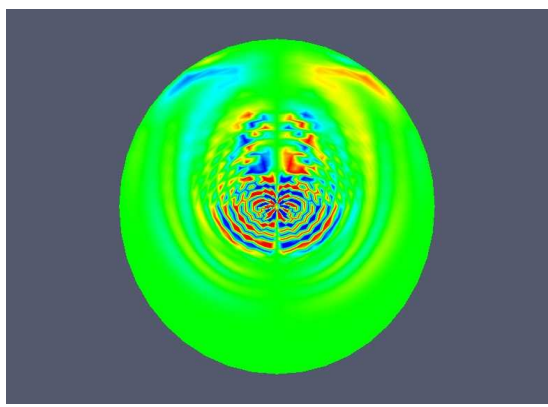

(a)

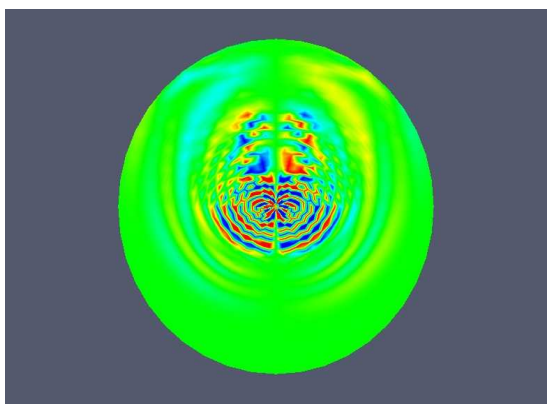

(b)

FIG. 8.5. Snapshot of wave propagation of $u_{x}$ at $t=0.16 \mathrm{~s}$ without (a) and with (b) exact absorbing boundary conditions.

\begin{tabular}{|c|c|}
\hline$N_{r} \times N_{\vartheta} \times N_{\phi}$ & $L_{2}$ norm errors \\
\hline $12 \times 12 \times 12$ & $7.32914 \times 10^{-8}$ \\
$18 \times 18 \times 18$ & $4.48387 \times 10^{-8}$ \\
$24 \times 24 \times 24$ & $2.04650 \times 10^{-8}$ \\
$30 \times 30 \times 30$ & $1.13200 \times 10^{-8}$ \\
$36 \times 36 \times 36$ & $9.24206 \times 10^{-9}$ \\
\hline
\end{tabular}

TABLE 8.2. $L_{2}$ norm errors of the numerical solutions at 1000 extrapolation time steps for various mesh sizes.

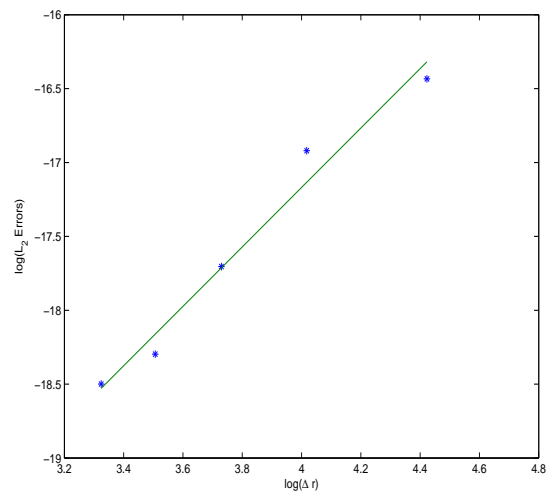

FIG. 8.6. A log-log plot for the $L_{2}$ norm errors.

as the waves have not yet reached the boundary. In figure 8.5(a) and figure 8.5(b), the snapshots of the waves at $t=0.16 \mathrm{~s}$ with and without the use of our boundary conditions are shown respectively. In figure 8.5(a) the boundary reflections are clearly seen while most of the boundary reflections are absorbed in figure $8.5(\mathrm{~b})$. This result shows that our boundary condition is effective in absorbing waves in almost all incident angles.

In the following, we will numerically test the convergence rate of the proposed computational scheme applied to the first example discussed above. In these calcula- 
tions, we consider the $L_{2}$ norm errors after 1000 time steps with various mesh sizes. To compute the errors, a reference solution is obtained on a fine grid. In table 8.2, we show the errors with various mesh sizes, and in figure 8.6 we show a log-log plot of the errors. In figure 8.6, the stars represent the errors and the line represents the least-square fitted line. We found that the slope of the line is 2.01. Thus we see that the rate of convergence of our computational scheme is 2. Similar convergence properties are also observed for the second example, so we omit the results.

\section{Conclusions}

In this paper, we have constructed a new exact nonreflecting boundary conditions for 3D poroelastic wave equations. The new exact nonreflecting boundary conditions given by (7.9) and (7.10) are applied on a spherical artificial surface which surrounds the computational domain. In the interior of the domain, the media may be inhomogeneous and contains complex structures, or may consists of several obstacles which are well separated from each other. Contrary to other common boundary conditions such as the one-way wave methods which are local on boundary, the exact conditions are nonlocal on boundary and are exact which ensures that the solution of the problem inside $\mathfrak{B}$ coincides with the solution of the original problem in the unbounded region. Moreover, the exact conditions are local in time and involve only first order time derivatives of the solutions. So they can be easily combined with standard numerical schemes for the computation of numerical solutions. Numerical experiments with the finite difference method show the effectiveness of the method derived in this paper and the ability to eliminate boundary reflections.

Acknowledgments. The authors are grateful to the editor Olor Runborg and the anonymous referees for their careful reading and constructive comments, which have greatly improved the paper. We also thank Prof. Z. Chen, Prof. J. Hong, Prof. J. Xu, and Prof. J. Zou for their important support in this research.

Appendix A.

Lemma A.1. For any $f(r) \in C^{1}$, we have

$$
\begin{aligned}
\nabla \times\left(f(r) \boldsymbol{V}_{n m}\right) & =-\frac{\sqrt{n(n+1)} f(r)}{r} Y_{n m} \hat{\boldsymbol{r}}-\frac{1}{r} \frac{\partial(r f)}{\partial r} \boldsymbol{U}_{n m}, \\
\hat{\boldsymbol{r}} \times \nabla \times\left(f(r) \boldsymbol{V}_{n m}\right) & =-\frac{1}{r} \frac{\partial(r f(r))}{\partial r} \boldsymbol{V}_{n m} \\
\hat{\boldsymbol{r}} \times \nabla \times\left(f(r) Y_{n m} \hat{\boldsymbol{r}}\right) & =\frac{\sqrt{n(n+1)} f(r)}{r} \boldsymbol{U}_{n m} \\
\nabla \cdot\left(f(r) \boldsymbol{U}_{n m}\right) & =-\frac{\sqrt{n(n+1)} f(r)}{r} Y_{n m} \\
\nabla \cdot \nabla\left(f(r) \boldsymbol{V}_{n m}\right) & =\left(\frac{\partial^{2} f(r)}{\partial r^{2}}+\frac{2}{r} \frac{\partial f(r)}{\partial r}-\frac{n(n+1)}{r^{2}} f(r)\right) \boldsymbol{V}_{n m} \\
\nabla \times \nabla \times\left(f(r) \boldsymbol{V}_{n m}\right) & =\left(\frac{n(n+1)}{r^{2}} f(r)-\frac{1}{r} \frac{\partial^{2}(r f(r))}{\partial r^{2}}\right) \boldsymbol{V}_{n m}
\end{aligned}
$$

Proof. To save space, we only prove (A.1). The others are can be proved similarly. Notice that

$$
\begin{aligned}
\nabla \times\left(f(r) \boldsymbol{V}_{n m}\right) & =\nabla \times\left(f(r) \hat{\boldsymbol{r}} \times \boldsymbol{U}_{n m}\right) \\
& =\left(\frac{\partial}{\partial r} \hat{\boldsymbol{r}}+\frac{1}{r} \frac{\partial}{\partial \vartheta} \hat{\boldsymbol{\vartheta}}+\frac{1}{r \sin \vartheta} \frac{\partial}{\partial \phi} \hat{\boldsymbol{\phi}}\right) \times\left(f(r) \hat{\boldsymbol{r}} \times \boldsymbol{U}_{n m}\right) .
\end{aligned}
$$


Calculating every term on the right side of (A.7), we get

$$
\begin{aligned}
& \frac{\partial}{\partial r} \hat{\boldsymbol{r}} \times\left(f(r) \hat{\boldsymbol{r}} \times \boldsymbol{U}_{n m}\right) \\
& =\frac{\partial}{\partial r} \hat{\boldsymbol{r}} \times\left[\frac{f(r)}{\sqrt{n(n+1)}}\left(\frac{-1}{\sin \vartheta} \frac{\partial Y_{n m}}{\partial \phi} \hat{\boldsymbol{\vartheta}}+\frac{\partial Y_{n m}}{\partial \vartheta} \hat{\boldsymbol{\phi}}\right)\right] \\
& =\frac{1}{\sqrt{n(n+1)}}\left[\frac{\partial}{\partial r}\left(\frac{-f(r)}{\sin \vartheta} \frac{\partial Y_{n m}}{\partial \phi}\right) \hat{\boldsymbol{r}} \times \hat{\boldsymbol{\vartheta}}+\frac{\partial}{\partial r}\left(f(r) \frac{\partial Y_{n m}}{\partial \vartheta}\right) \hat{\boldsymbol{r}} \times \hat{\boldsymbol{\phi}}\right] \\
& =\frac{1}{\sqrt{n(n+1)}}\left[\frac{\partial}{\partial r}\left(\frac{-f(r)}{\sin \vartheta} \frac{\partial Y_{n m}}{\partial \phi}\right) \hat{\boldsymbol{\phi}}-\frac{\partial}{\partial r}\left(f(r) \frac{\partial Y_{n m}}{\partial \vartheta}\right) \hat{\boldsymbol{\vartheta}}\right] \\
& =-\frac{1}{\sqrt{n(n+1)}}\left(\frac{\partial Y_{n m}}{\partial \vartheta} \hat{\boldsymbol{\vartheta}}+\frac{1}{\sin \vartheta} \frac{\partial Y_{n m}}{\partial \phi} \hat{\boldsymbol{\phi}}\right) \frac{\partial f(r)}{\partial r} \\
& =-\frac{\partial f(r)}{\partial r} \boldsymbol{U}_{n m}, \\
& \frac{1}{r} \frac{\partial}{\partial \vartheta} \hat{\boldsymbol{\vartheta}} \times\left(f(r) \hat{\boldsymbol{r}} \times \boldsymbol{U}_{n m}\right) \\
& =\frac{1}{r} \frac{\partial}{\partial \vartheta} \hat{\boldsymbol{\vartheta}} \times\left[\frac{f(r)}{\sqrt{n(n+1)}}\left(\frac{-1}{\sin \vartheta} \frac{\partial Y_{n m}}{\partial \phi} \hat{\boldsymbol{\vartheta}}+\frac{\partial Y_{n m}}{\partial \vartheta} \hat{\boldsymbol{\phi}}\right)\right] \\
& =\frac{f(r)}{r \sqrt{n(n+1)}}\left[\hat{\boldsymbol{\vartheta}} \times \frac{\partial}{\partial \vartheta}\left(\frac{-1}{\sin \vartheta} \frac{\partial Y_{n m}}{\partial \phi} \hat{\boldsymbol{\vartheta}}\right)+\hat{\boldsymbol{\vartheta}} \times \frac{\partial}{\partial \vartheta}\left(\frac{\partial Y_{n m}}{\partial \vartheta} \hat{\boldsymbol{\phi}}\right)\right] \\
& =\frac{f(r)}{r \sqrt{n(n+1)}}\left(-\frac{1}{\sin \vartheta} \frac{\partial Y_{n m}}{\partial \phi} \hat{\boldsymbol{\phi}}+\frac{\partial^{2} Y_{n m}}{\partial \vartheta^{2}} \hat{\boldsymbol{r}}\right) \text {, } \\
& \frac{1}{r \sin \vartheta} \frac{\partial}{\partial \phi} \hat{\boldsymbol{\phi}} \times\left(f(r) \hat{\boldsymbol{r}} \times \boldsymbol{U}_{n m}\right) \\
& =\frac{1}{r \sin \vartheta} \frac{\partial}{\partial \phi} \hat{\boldsymbol{\phi}} \times\left[\frac{f(r)}{\sqrt{n(n+1)}}\left(\frac{-1}{\sin \vartheta} \frac{\partial Y_{n m}}{\partial \phi} \hat{\boldsymbol{\vartheta}}+\frac{\partial Y_{n m}}{\partial \vartheta} \hat{\boldsymbol{\phi}}\right)\right] \\
& =\frac{f(r)}{r \sin \vartheta \sqrt{n(n+1)}}\left[\hat{\boldsymbol{\phi}} \times \frac{\partial}{\partial \phi}\left(\frac{-1}{\sin \vartheta} \frac{Y_{n m}}{\partial \phi} \hat{\boldsymbol{\vartheta}}\right)+\hat{\boldsymbol{\phi}} \times \frac{\partial}{\partial \phi}\left(\frac{Y_{n m}}{\partial \vartheta} \hat{\boldsymbol{\phi}}\right)\right] \\
& =\frac{f(r)}{r \sin \vartheta \sqrt{n(n+1)}}\left[\left(\frac{1}{\sin \vartheta} \frac{\partial^{2} Y_{n m}}{\partial \phi^{2}}+\cos \vartheta \frac{\partial Y_{n m}}{\partial \vartheta}\right) \hat{\boldsymbol{r}}-\sin \vartheta \frac{\partial Y_{n m}}{\partial \vartheta} \hat{\boldsymbol{\vartheta}}\right] \text {. }
\end{aligned}
$$

Inserting (A.8)-(A.10) into (A.7) yields

$$
\begin{aligned}
& \nabla \times\left(f(r) \boldsymbol{V}_{n m}\right) \\
= & \frac{f(r)}{r \sqrt{n(n+1)}}\left(\frac{1}{\sin \vartheta} \frac{\partial}{\partial \vartheta}\left(\sin \vartheta \frac{\partial Y_{n m}}{\partial \vartheta}\right)+\frac{1}{\sin ^{2} \vartheta} \frac{\partial^{2} Y_{n m}}{\partial \phi^{2}}\right) \hat{\boldsymbol{r}}-\frac{1}{r} \frac{\partial(r f)}{\partial r} \boldsymbol{U}_{n m} .
\end{aligned}
$$

Since $Y_{n m}$ is the eigenvector of Beltrami operator $\mathcal{B}$ with eigenvalue $-n(n+1)$,

$$
\mathcal{B} Y_{n m}=-n(n+1) Y_{n m},
$$

where $\mathcal{B}$ is defined by

$$
\mathcal{B} \equiv \frac{1}{\sin \vartheta} \frac{\partial}{\partial \vartheta}\left(\sin \vartheta \frac{\partial}{\partial \vartheta}\right)+\frac{1}{\sin ^{2} \vartheta} \frac{\partial^{2}}{\partial \phi^{2}}
$$


Thus

$$
\nabla \times\left(f(r) \boldsymbol{V}_{n m}\right)=-\frac{\sqrt{n(n+1)} f(r)}{r} Y_{n m} \hat{\boldsymbol{r}}-\frac{1}{r} \frac{\partial(r f)}{\partial r} \boldsymbol{U}_{n m}
$$

\section{Appendix B.}

In the following, we give the proof of Lemma 4.1.

Proof. We will only give a proof for (4.6). The proof of (4.7) is similar. For $n=0$, the conclusion is obvious. For $n=1$, from (2.20) we have

$$
\begin{aligned}
\text { LHS }:=r \int_{\infty}^{r}\{(\stackrel{\circ}{M} \lambda & \left.+2 \stackrel{\circ}{M} \mu-\alpha \rho_{f} M\right)\left(\frac{\partial^{2} h_{n m}^{1}}{\partial r^{2}}+\frac{2}{s} \frac{\partial h_{n m}^{1}}{\partial r}-\frac{2}{s^{2}} h_{n m}^{1}\right) \\
& \left.+\left(\alpha \stackrel{\circ}{M} M-\rho_{f} M\right)\left(\frac{\partial^{2} h_{n m}^{2}}{\partial r^{2}}+\frac{2}{s} \frac{\partial h_{n m}^{2}}{\partial r}-\frac{2}{s^{2}} h_{n m}^{2}\right)\right\} d s
\end{aligned}
$$

and

$$
\begin{aligned}
\text { RHS }:=(\stackrel{\circ}{M} \lambda & \left.+2 \stackrel{\circ}{M} \mu-\alpha \rho_{f} M\right)\left(r \frac{\partial h_{n m}^{1}}{\partial r}+2 h_{n m}^{1}\right) \\
& +\left(\alpha \stackrel{\circ}{M} M-\rho_{f} M\right)\left(r \frac{\partial h_{n m}^{2}}{\partial r}+2 h_{n m}^{2}\right) .
\end{aligned}
$$

It is then sufficient to show

$$
r \int_{\infty}^{r}\left(\frac{\partial^{2} h_{n m}^{i}}{\partial r^{2}}+\frac{2}{s} \frac{\partial h_{n m}^{i}}{\partial r}-\frac{2}{s^{2}} h_{n m}^{i}\right) d s=r \frac{\partial h_{n m}^{i}}{\partial r}+2 h_{n m}^{i}, \quad i=1,2 .
$$

With this, the LHS is equal to the RHS, and the conclusion follows immediately.

By assumption, the initial data $h_{n m}^{i}$ and $\partial_{r} h_{n m}^{i}(i=1,2)$ have compact support, so at any fixed time $t, h_{n m}^{i}$ and $\partial_{r} h_{n m}^{i}(i=1,2)$ vanish for $s \rightarrow \infty$. Thus

$$
\begin{aligned}
r \int_{\infty}^{r}\left(\frac{\partial^{2} h_{n m}^{i}}{\partial r^{2}}+\frac{2}{s} \frac{\partial h_{n m}^{i}}{\partial r}-\frac{2}{s^{2}} h_{n m}^{i}\right) d s & =\left.r\left(\frac{\partial}{\partial r} h_{n m}^{i}(s, t)+\frac{2}{s} h_{n m}^{i}(s, t)\right)\right|_{s=\infty} ^{r} \\
& =r \frac{\partial h_{n m}^{i}}{\partial r}+2 h_{n m}^{i}, \quad i=1,2 .
\end{aligned}
$$

This means that the conclusion holds for $n=1$.

For $n=2$,

$$
\begin{aligned}
\text { LHS }:=r \int_{\infty}^{r} \frac{s^{2}-r^{2}}{2 s} & {\left[\left(\stackrel{\circ}{M} \lambda+2 \stackrel{\circ}{M} \mu-\alpha \rho_{f} M\right)\left(\frac{\partial^{2}}{\partial r^{2}}+\frac{2}{s} \frac{\partial}{\partial r}-\frac{6}{s^{2}}\right) h_{n m}^{1}\right.} \\
+ & \left.\left(\alpha \stackrel{\circ}{M} M-\rho_{f} M\right)\left(\frac{\partial^{2}}{\partial r^{2}}+\frac{2}{s} \frac{\partial}{\partial r}-\frac{6}{s^{2}}\right) h_{n m}^{2}\right] d s
\end{aligned}
$$

and

$$
\begin{aligned}
\text { RHS }:=(\stackrel{\circ}{M} \lambda & \left.+2 \stackrel{\circ}{M} \mu-\alpha \rho_{f} M\right) \frac{\partial^{2}}{\partial r^{2}}\left(r \int_{\infty}^{r} \frac{\left(s^{2}-r^{2}\right) h_{n m}^{1}}{2 s} d s\right) \\
& +\left(\alpha \stackrel{\circ}{M} M-\rho_{f} M\right) \frac{\partial^{2}}{\partial r^{2}}\left(r \int_{\infty}^{r} \frac{\left(s^{2}-r^{2}\right) h_{n m}^{2}}{2 s} d s\right) .
\end{aligned}
$$


Comparison of LHS and RHS shows that if the following equality holds $(i=1,2)$,

$$
\frac{\partial^{2}}{\partial r^{2}}\left(r \int_{\infty}^{r} \frac{\left(s^{2}-r^{2}\right) h_{n m}^{i}}{2 s} d s\right)=r \int_{\infty}^{r} \frac{s^{2}-r^{2}}{2 s}\left(\frac{\partial^{2}}{\partial r^{2}}+\frac{2}{s} \frac{\partial}{\partial r}-\frac{6}{s^{2}}\right) h_{n m}^{i} d s,
$$

then the conclusion will hold. Using the fact that

$$
\frac{\partial^{2}}{\partial r^{2}}\left(r \int_{\infty}^{r} \frac{\left(s^{2}-r^{2}\right) h_{n m}^{i}}{2 s} d s\right)=-3 r \int_{\infty}^{r} \frac{h_{n m}^{i}}{s} d s-r h_{n m}^{i}, \quad i=1,2,
$$

and integrating by parts, we annihilate the derivatives $\partial_{r r} h_{n m}^{i}$ and $\partial_{r} h_{n m}^{i}$ on the right side of (B.7) and obtain

$$
r \int_{\infty}^{r} \frac{s^{2}-r^{2}}{2 s}\left(\frac{\partial^{2}}{\partial r^{2}}+\frac{2}{s} \frac{\partial}{\partial r}-\frac{6}{s^{2}}\right) h_{n m}^{i} d s=-3 r \int_{\infty}^{r} \frac{h_{n m}^{i}}{s} d s-r h_{n m}^{i}, i=1,2 .
$$

Thus (4.6) holds for $n=2$.

For $n \geq 3$, similar analysis shows that the conclusion is equivalent to the following equality:

$$
\begin{aligned}
& (4 n-2) r^{3} \int_{\infty}^{r} \frac{\left(s^{2}-r^{2}\right)^{n-3}}{(2 s)^{n-1}(n-2) !} h_{n m}^{i} d s-6 r \int_{\infty}^{r} \frac{\left(s^{2}-r^{2}\right)^{n-3} s^{2}}{(2 s)^{n-1}(n-2) !} h_{n m}^{i} d s \\
= & r \int_{\infty}^{r} \frac{\left(s^{2}-r^{2}\right)^{n-1}}{(2 s)^{n-1}(n-1) !}\left(\frac{\partial^{2}}{\partial r^{2}}+\frac{2}{s} \frac{\partial}{\partial r}-\frac{n(n+1)}{s^{2}}\right) h_{n m}^{i} d s, \quad i=1,2 .
\end{aligned}
$$

We annihilate the derivatives $\partial_{r r} h_{n m}^{i}$ and $\partial_{r} h_{n m}^{i}$ on the right side of (B.10) and get

$$
\begin{aligned}
& r \int_{\infty}^{r} \frac{\left(s^{2}-r^{2}\right)^{n-1}}{(2 s)^{n-1}(n-1) !} \frac{\partial^{2} h_{n m}^{i}}{\partial r^{2}} d s \\
= & -2 r \int_{\infty}^{r} \frac{\left(s^{2}+r^{2}\right)\left(s^{2}-r^{2}\right)^{n-2}}{(2 s)^{n}(n-2) !} \frac{\partial h_{n m}^{i}}{\partial r} d s \\
= & r \int_{\infty}^{r} \frac{\left(s^{2}-r^{2}\right)^{n-3}}{(2 s)^{n-1}(n-2) !}\left(2(n-1) s^{2}+2(n-3) r^{2}-n\left(s^{2}-\frac{r^{4}}{s^{2}}\right)\right) h_{n m}^{i} d s
\end{aligned}
$$

and

$$
\begin{aligned}
& r \int_{\infty}^{r} \frac{\left(s^{2}-r^{2}\right)^{n-1}}{(2 s)^{n-1}(n-1) !} \frac{2}{s} \frac{\partial h_{n m}^{i}}{\partial r} d s \\
= & -\int_{\infty}^{r} \frac{\left(s^{2}-r^{2}\right)^{n-3}}{(2 s)^{n-1}(n-2) !}\left(\frac{2 n-4}{n-1} s^{2}+\frac{4}{n-1} r^{2}-\frac{2 n}{n-1} \frac{r^{4}}{s^{2}}\right) h_{n m}^{i} d s
\end{aligned}
$$

Inserting (B.11) and (B.12) into the right side of (B.10), we see that (B.10) holds. Therefore the conclusion holds for $n \geq 3$.

\section{REFERENCES}

[1] B. Alpert, L. Greengard, and T. Hagstrom, Rapid evaluation of nonreflecting boundary kernels for time-domain wave propagation, SIAM J. Numer. Anal., 37, 1138-1164, 2000.

[2] B. Alpert, L. Greengard, and T. Hagstrom, Nonreflecting boundary conditions for the timedependent wave equation, J. Comput. Phys., 180, 270-296, 2002.

[3] D. Appelö and T. Hagstrom, A general perfectly matched layer model for hyperbolic-parabolic systems, SIAM J. Sci. Comput., 31, 3301-3323, 2009. 
[4] D. Appelö, T. Hagstrom, and G. Kreiss, Perfectly matched layers for hyperbolic systems: General formulation, well-posedness, and stability, SIAM J. Appl. Math., 67, 1-23, 2006.

[5] A. Bayliss and E. Turkel, Radiation boundary conditions for wave-like equations, Commun. Pure Appl. Math., 33, 707-725, 1980.

[6] E. Bécache, S. Fauqueux, and P. Joly, Stability of perfectly matched layers, group velocities and anisotropic waves, J. Comput. Phys., 188, 399-433, 2003.

[7] E. Bécache, D. Givoli, and T. Hagstrom, High-order absorbing boundary conditions for anisotropic and convective wave equations, J. Comput. Phys., 229, 1099-1129, 2010.

[8] E. Bécache and P. Joly, On the analysis of Berenger's perfectly matched layers for Maxwell's equations, Math. Model Numer. Anal., 36, 87-119, 2002.

[9] E. Bécache, P. Petropoulos, and S. Gedney, On the long-time behavior of unsplit perfectly matched layers, IEEE Trans. Antennas Prop., 54, 1335-1342, 2004.

[10] J.P. Bérenger, A perfectly matched layer for the absorption of electromagetic waves, J. Comput. Phys., 114, 185-200, 1994.

[11] A. Bermúdez, L. Hervella-Nieto, A. Prieto, and R. Rodríguez, An exact bounded perfectly matched layer for time-harmonic scattering problems, SIAM J. Sci. Comput., 30, 312-338, 2007.

[12] M.A. Biot, Theory of propagation of elastic waves in a fluid-saturated porous solid. I. Lowfrequency range, J. Acoust. Soc. Am., 28, 168-178, 1956.

[13] C. Cerjan, D. Kosloff, R. Kosloff, and M. Reshef, A nonreflecting boundary condition for discrete acoustic and elastic wave equations, Geophysics, 50, 705-708, 1985.

[14] Z. Chen and X. Liu, An adaptive perfectly matched layer technique for time-harmonic scattering problems, SIAM J. Numer. Anal., 43, 645-671, 2005.

[15] E.T. Chung and B. Engquist, Optimal discontinuous Galerkin methods for wave propagation, SIAM J. Numer. Anal., 44, 2131-2158, 2006.

[16] E.T. Chung and B. Engquist, Optimal discontinuous Galerkin methods for the acoustic wave equation in higher dimensions, SIAM J. Numer. Anal., 47, 3820-3848, 2009.

[17] R. Clayton and B. Engquist, Absorbing boundary conditions for acoustic and elastic wave equations, Bull. Seism. Soc. Am., 67, 1529-1540, 1977.

[18] F. Collino and C. Tsogka, Application of the perfectly matched absorbing layer model to the linear elastodynamic problem in anisotropic heterogeneous media, Geophysics, 66, 294-307, 2001.

[19] D. Colton and R. Kress, Inverse Acoustic and Electromagnetic Scatting Theory, SpringerVerlag, Berlin, New York, 1992.

[20] J.R. Driscoll, D.M. Healy Jr., and D.N. Rockmore, Fast discrete polynomial transforms with applications to data analysis for distance transitive graphs, SIAM J. Comput., 26, 10661099, 1997.

[21] J. Diaz and P. Joly, An analysis of higher order boundary conditions for the wave equation, SIAM J. Appl. Math., 65, 1547-1575, 2005.

[22] B. Engquist and A. Majda, Absorbing boundary conditions for the numerical simulation of waves, Math. Comput., 31, 629-651, 1977.

[23] B. Engquist and A. Majda, Radiation boundary conditions for acoustic and elastic wave calculations, Commun. Pure. Appl. Math., 32, 313-357, 1979.

[24] D. Givoli, High-order local non-reflecting boundary conditions: A review, Wave Motion, 39, 319-326, 2004.

[25] D. Givoli and D. Cohen, Nonreflecting boundary constions based on Kirchhoff-type formulae, J. Comput. Phys., 117, 102-113, 1995.

[26] D. Givoli and B. Neta, High-order non-reflecting boundary scheme for time-dependent waves, J. Comput. Phys., 186, 24-46, 2003.

[27] D. Givoli, T. Hagstrom, and I. Patlashenko, Finite element formulation with high-order absorbing boundary conditions for time-dependent waves, Comput. Meth. Appl. Mech. Engin., 195, 3666-3690, 2006.

[28] M.J. Grote and J.B. Keller, Exact nonreflecting boundary conditions for the time dependent wave equation, SIAM J. Appl. Math., 55, 280-297, 1995.

[29] M.J. Grote and J.B. Keller, Nonreflecting boundary conditions for time dependent scattering, J. Comput. Phys., 139, 52-65, 1996.

[30] M.J. Grote and J.B. Keller, Nonreflecting boundary conditions for Maxwell's equations, J. Comput. Phys., 139, 327-342, 1998.

[31] M.J. Grote and J.B. Keller, Exact nonreflecting boundary condition for elastic waves, SIAM J. Appl. Math., 60, 803-819, 2000.

[32] B.D. Gupta, Mathematical Physics, Vikas Publishing House PVT LTD, India, 1978.

[33] T. Hagstrom, Radiation boundary conditions for the numerical simulation of waves, Acta. 
Numer., 8, 47-106, 1999.

[34] T. Hagstrom, and S.I. Hariharan, A formulation of asymptotic and exact boundary conditions using local operators, Appl. Numer. Math., 27, 403-416, 1998.

[35] T. Hagstrom, S.I. Hariharan, and D. Thompson, High-order radiation boundary conditions for wave convective wave equation in exterior domains, SIAM J. Sci. Comput., 25, 1088-1101, 2003.

[36] T. Hagstrom, A. Mar-Or, and D. Givoli, High-order local absorbing boundary conditions for the wave equations: Extension and improvements, J. Comput. Phys., 227, 3322-3357, 2008.

[37] T. Hagstrom, and T. Warburton, A new auxiliary variable formulation of high-order radiation boundary conditions: Corner compatibiltiy conditions and extensions to first-order systems, Wave Motion, 39, 327-338, 2004.

[38] T. Hagstrom and T. Warburton, Complete radiation boundary conditions: Minimizing the long time error growth of local methods, SIAM J. Numer. Anal., 47, 3678-3704, 2009.

[39] T. Hagstrom, T. Warburtonb, and D. Givolic, Radiation boundary conditions for timedependent waves based on complete plane wave expansions, J. Comput. Appl. Math., 234, 1988-1995, 2010.

[40] L. Halpern and L.N. Trefethen, Wide-angle one-way wave equations, J. Acoust. Soc. Am., 84, 1397-1404, 1988.

[41] F. Hastings, J.B. Schneider, and S.L. Broschat, Application of the perfectly matched layer $(P M L)$ absorbing boundary condition to elastic wave propagation, J. Acoust. Soc. Am., 100, 3061-3069, 1996.

[42] R.L. Higdon, Absorbing boundary conditions for difference approximations to the multidimensional wave equation, Math. Comput., 47, 437-459, 1986.

[43] R.L. Higdon, Radiation boundary conditions for elastic wave propagation, SIAM J. Numer. Anal., 27, 831-870, 1990.

[44] R.L. Higdon, Absorbing boundary conditions for acoustic and elastic wave equations in stratified media, J. Comput. Phys., 101, 386-418, 1992.

[45] R.L. Higdon, Radiation boundary conditions for dispersive waves, SIAM J. Numer. Anal., 31, 64-100, 1994.

[46] J.B. Keller and D. Givoli, Exact non-reflecting boundary conditions, J. Comput. Phys., 82, 172-192, 1989.

[47] R. Kosloff and D. Kosloff, Absorbing boundaries for wave propagation problems, J. Comput. Phys., 63, 363-367, 1986.

[48] D. Komatisch and J. Tromp, A perfectly matched layer absorbing boundary condition for the second-order seismic wave equation, Geophys. J. Internat., 154, 146-153, 2003.

[49] E.L. Lindman, 'Free-space' boundary conditions for the time dependent wave equation, J. Comput. Phys., 18, 66-78, 1975.

[50] K.D. Mahrer, An empirical study of instability and improvement of absorbing boundary conditions for elastic wave equations, Geophysics, 51, 1499-1501, 1986.

[51] D. Potts, Fast algorithms for discrete polynomial transforms on arbitrary grids, Lin. Alg. Appl., 366, 353-370, 2003.

[52] D. Rabinovich, D. Givoli, and E. Bécache, Comparison of high-order absorbing boundary conditions and perfectly matched layers in the frequency domain, Int. J. Numer. Meth. Biomed. Engin, 26, 1351-1369, 2010.

[53] A. Simone and S. Hestholm, Instabilities in applying absorbing boundary conditions to highorder seismic modeling algorithms, Geophysics, 63, 1017-1023, 1998.

[54] I.L. Sofronov, Artificial boundary conditions of absolute transparency for two- and three- dimensional external time-dependent scattering problems, Euro. J. Appl. Math., 9, 561-588, 1998.

[55] L. Ting and M.J. Miksis, Exact boundary conditions for scattering problems, J. Acoust. Soc. Am., 80, 1825-1827, 1986.

[56] L.N. Trefethen and L. Halpern, Well-posedness of one-way wave equations and absorbing boundary condition, Math. Comput., 47, 421-435, 1986.

[57] L. Wagatha, Approximation of pseudodifferential operators in absorbing boundary conditions for hyperbolic equations, Numer. Math., 42, 51-64, 1983.

[58] Y.Q. Zeng, J.Q. He, and Q.L. Liu, The application of the perfectly matched layer in numerical modeling of wave propagation in poroelastic media, Geophysics, 66, 1258-1266, 2001.

[59] Y.Q. Zeng and Q.L. Liu, A staggered-grid finite-difference method with perfectly matched layers for poroelastic wave equations, J. Acoust. Soc. Am., 109, 2571-2580, 2011. 